\title{
The Chemistry of ortho-(Diarylphosphino)aryl Isocyanides
}

Lujun Zhang ${ }^{\mathrm{a}}$, Wenfei Yü, Changchun Liu ${ }^{\mathrm{a}}$, Youzhi Xu ${ }^{\mathrm{a}}$, Zheng Duan*a,

Francois Mathey ${ }^{\star a, b}$

a) College of Chemistry and Molecular Engineering, International Phosphorus Laboratory, International Joint Research Laboratory for Functional Organophosphorus Materials of Henan Province, Zhengzhou University, Zhengzhou 450001, P. R. China;

b) Division of Chemistry \& Biological Chemistry, Nanyang Technological University, 21

Nanyang Link, Singapore 637371

duanzheng@zzu.edu.cn,FMathey@ntu.edu.sg

\section{Contents:}

1) General Information

2) Synthesis and Characterization Data of All New Compounds

1a-1c, 4a-4c, 5a-5c, 6a-6c, 9a-9b, 10a-10b, 11-13

3) X-ray Crystallographic Studies of $\mathbf{5 b} ; \mathbf{1 0 a} ; \mathbf{1 0 b} ; \mathbf{1 1} \mathbf{1 2} ; 13$

4) Scanned ${ }^{31} \mathrm{P}$ NMR, ${ }^{1} \mathrm{H}$ NMR and ${ }^{13} \mathrm{C}$ NMR Spectra of All

New Compounds 1a-1c, 3a-3c ( ${ }^{31} \mathbf{P}$ NMR), 4a-4c, 5a-5c, 6a-6c, 9a-9b, 10a-10b ( ${ }^{1}$ H NMR), 11 ( ${ }^{1}$ H NMR), 12-13 


\section{1) General Information}

All reactions were performed under nitrogen using solvents dried by standard methods. ${ }^{1} \mathrm{H},{ }^{13} \mathrm{C}$ and ${ }^{31} \mathrm{P}$ NMR spectra were recorded on Bruker 300 and $400 \mathrm{MHz}$ spectrometer. Chemical shifts are expressed in ppm from internal TMS $\left({ }^{1} \mathrm{H}\right.$ and $\left.{ }^{13} \mathrm{C}\right)$. All coupling constants $(J$ values) are reported in hertz $(\mathrm{Hz})$. HRMS spectra were obtained on a Water Q - Tof Premier MS. Element analytic data were obtained on a Thermo Electron Corporation flash EA 1112 element spectrometer. Melting Point: heating rate: $4^{\circ} \mathrm{C} / \mathrm{min}$, the thermometer was not corrected. Silica gel $(230-400$ mesh) was used for the chromatographic separations. 2a and $\mathbf{2 b}$ were prepared according to literature methods ${ }^{[1]}$. Commercially available reagents were used without further purification.

\section{2) Synthesis and Characterization Data of All New Compounds}

\section{$1 a-1 c, 4 a-4 c, 5 a-5 c, 6 a-6 c, 9 a-9 b, 10 a-10 b, 11-13$}<smiles>[R]c1ccc(Br)c([N+]#[C-])c1</smiles>

2a $\mathrm{R}=\mathrm{H}$

2b R=Me<smiles>[R]c1ccc(Cl)c(N=C)c1</smiles>
$-78^{\circ} \mathrm{C}, 1 \mathrm{~h}$ 
at $-78^{\circ} \mathrm{C}$ for $1 \mathrm{~h}, \mathrm{Ar}_{2} \mathrm{PCl}(12.6 \mathrm{mmol})$ in anhydrous $\mathrm{THF}(10 \mathrm{~mL})$ was added dropwise. The mixture was kept at $-78^{\circ} \mathrm{C}$ for $1 \mathrm{~h}$ and then warmed to room temperature. After $3 \mathrm{~h}$, the reaction was completed. The mixture was diluted with diethyl ether, the organic phase washed with water, brine and dried over anhydrous $\mathrm{Na}_{2} \mathrm{SO}_{4}$. The solvents were removed under reduced pressure to give a crude product, which was purified by recrystallization (dichloromethane and hexane) at about $10^{\circ} \mathrm{C}$ and to give a yellow solid.

$1 \mathrm{a}$ (m.p. $125-126{ }^{\circ} \mathrm{C}, 2361 \mathrm{mg}, 65 \%$ yield): ${ }^{31} \mathrm{P}$ NMR (121MHz, $\left.\mathrm{CDCl}_{3}\right)$ : $\delta=-11.8 \mathrm{ppm} ;{ }^{1} \mathrm{H} \mathrm{NMR}\left(300 \mathrm{MHz}, \mathrm{CDCl}_{3}\right): \delta=6.88-6.92(\mathrm{~m}, 1 \mathrm{H}), 7.30-7$. $42(\mathrm{~m}, 13 \mathrm{H}) \mathrm{ppm} ;{ }^{13} \mathrm{C} \mathrm{NMR}\left(101 \mathrm{MHz}, \mathrm{CDCl}_{3}\right): \delta=127.30\left(\mathrm{~d}, J_{\mathrm{CP}}=2.0 \mathrm{~Hz}\right.$, $\mathrm{CH}), \quad 128.85\left(\mathrm{~d}, J_{\mathrm{CP}}=8.0 \mathrm{~Hz}, 4 \mathrm{CH}\right), \quad 129.28(\mathrm{~s}, \mathrm{CH}), \quad 129.45(\mathrm{~s}, \quad 2 \mathrm{CH})$, $129.49(\mathrm{~s}, \mathrm{CH}), 133.41(\mathrm{~s}, \mathrm{CH}), 134.18\left(\mathrm{~d}, J_{\mathrm{CP}}=21.1 \mathrm{~Hz}, 4 \mathrm{CH}\right), 134.30(\mathrm{~d}$, $\left.J_{\mathrm{CP}}=10.1 \mathrm{~Hz}, 2 \mathrm{C}\right), 135.66\left(\mathrm{~d}, J_{\mathrm{CP}}=19.1 \mathrm{~Hz}, \mathrm{C}\right), 168.12(\mathrm{~s}, \mathrm{C}) \mathrm{ppm}$. One carbon is missing due to the overlap. HRMS calcd for $\mathrm{C}_{19} \mathrm{H}_{15} \mathrm{NP}[\mathrm{M}+\mathrm{H}]^{+}$: 288.0937; found :288.0934.

1b(m.p. 125-126 ${ }^{\circ} \mathrm{C}, 2361 \mathrm{mg}, 60 \%$ yield): ${ }^{31} \mathrm{P}$ NMR (121MHz, $\mathrm{CDCl}_{3}$ ): $\delta=-13.2 \mathrm{ppm} ;{ }^{1} \mathrm{H}$ NMR $\left(300 \mathrm{MHz}, \mathrm{CDCl}_{3}\right): \delta=2.37(\mathrm{~s}, 3 \mathrm{H}), 6.79(\mathrm{dd}, J=3.3$, $7.8 \mathrm{~Hz}, 1 \mathrm{H}), 7.12(\mathrm{~d}, J=8.1 \mathrm{~Hz}, 1 \mathrm{H}), 7.25(\mathrm{brs}, 1 \mathrm{H}), \quad 7.30-7.41(\mathrm{~m}$, $10 \mathrm{H}) \mathrm{ppm} ;{ }^{13} \mathrm{C} \mathrm{NMR}\left(75 \mathrm{MHz}, \mathrm{CDCl}_{3}\right): \delta=20.94\left(\mathrm{~s}, \mathrm{CH}_{3}\right), 127.85(\mathrm{~d}$, $\left.J_{\mathrm{CP}}=2.0 \mathrm{~Hz}, \mathrm{CH}\right), 128.76\left(\mathrm{~d}, J_{\mathrm{CP}}=7.5 \mathrm{~Hz}, 4 \mathrm{CH}\right), 129.31(\mathrm{~s}, 2 \mathrm{CH}), 130.27(\mathrm{~s}$, $\mathrm{CH}), 131.87\left(\mathrm{~d}, J_{\mathrm{CP}}=18.1 \mathrm{~Hz}, \mathrm{C}\right), 133.42(\mathrm{~s}, \mathrm{CH}), 134.05\left(\mathrm{~d}, J_{\mathrm{CP}}=20.4 \mathrm{~Hz}\right.$, 
$4 \mathrm{CH}), 134.65\left(\mathrm{~d}, J_{\mathrm{CP}}=9.8 \mathrm{~Hz}, 2 \mathrm{C}\right), 140.14(\mathrm{~s}, \mathrm{C}), 167.35(\mathrm{~s}, \mathrm{C}, \mathrm{NC}) \mathrm{ppm}$. One carbon is missing due to the overlap. HRMS calcd for $\mathrm{C}_{20} \mathrm{H}_{17} \mathrm{NP}$ [M $+\mathrm{H}]^{+}:$302.1093; found : 302.1099 .

1c(m.p. $98-100{ }^{\circ} \mathrm{C}, 3148 \mathrm{mg}, 79 \%$ yield): ${ }^{31} \mathrm{P} \mathrm{NMR}\left(121 \mathrm{MHz}, \mathrm{CDCl}_{3}\right): \delta=$ -13.5ppm; ${ }^{1} \mathrm{H}$ NMR $\left(300 \mathrm{MHz}, \mathrm{CDCl}_{3}\right): \delta=2.39(\mathrm{~s}, 6 \mathrm{H}), 6.89-6.93(\mathrm{~m}$, 1H), 7.19-7.41 (m, 11H)ppm; ${ }^{13} \mathrm{C}$ NMR $\left(75 \mathrm{MHz}, \mathrm{CDCl}_{3}\right): \delta=21.42(\mathrm{~s}$, $\left.2 \mathrm{CH}_{3}\right), \quad 127.20\left(\mathrm{~d}, J_{\mathrm{CP}}=1.5 \mathrm{~Hz}, \mathrm{CH}\right), \quad 129.16(\mathrm{~s}, \mathrm{CH}), \quad 129.21(\mathrm{~s}, \mathrm{CH})$, $129.65\left(\mathrm{~d}, J_{\mathrm{CP}}=8.3 \mathrm{~Hz}, 4 \mathrm{CH}\right), 130.86\left(\mathrm{~d}, J_{\mathrm{CP}}=9.0 \mathrm{~Hz}, 2 \mathrm{C}\right), 133.23(\mathrm{~s}, \mathrm{CH})$, $134.26\left(\mathrm{~d}, J_{\mathrm{CP}}=20.2 \mathrm{~Hz}, 4 \mathrm{CH}\right), 136.34\left(\mathrm{~d}, J_{\mathrm{CP}}=20.2 \mathrm{~Hz}, \mathrm{C}\right), 139.45(\mathrm{~s}, 2 \mathrm{C})$, 168.01(s, C)ppm. One carbon is missing due to the overlap. HRMS calcd for $\mathrm{C}_{21} \mathrm{H}_{19} \mathrm{NP}[\mathrm{M}+\mathrm{H}]^{+}:$316.1250; found : 316.1257 .

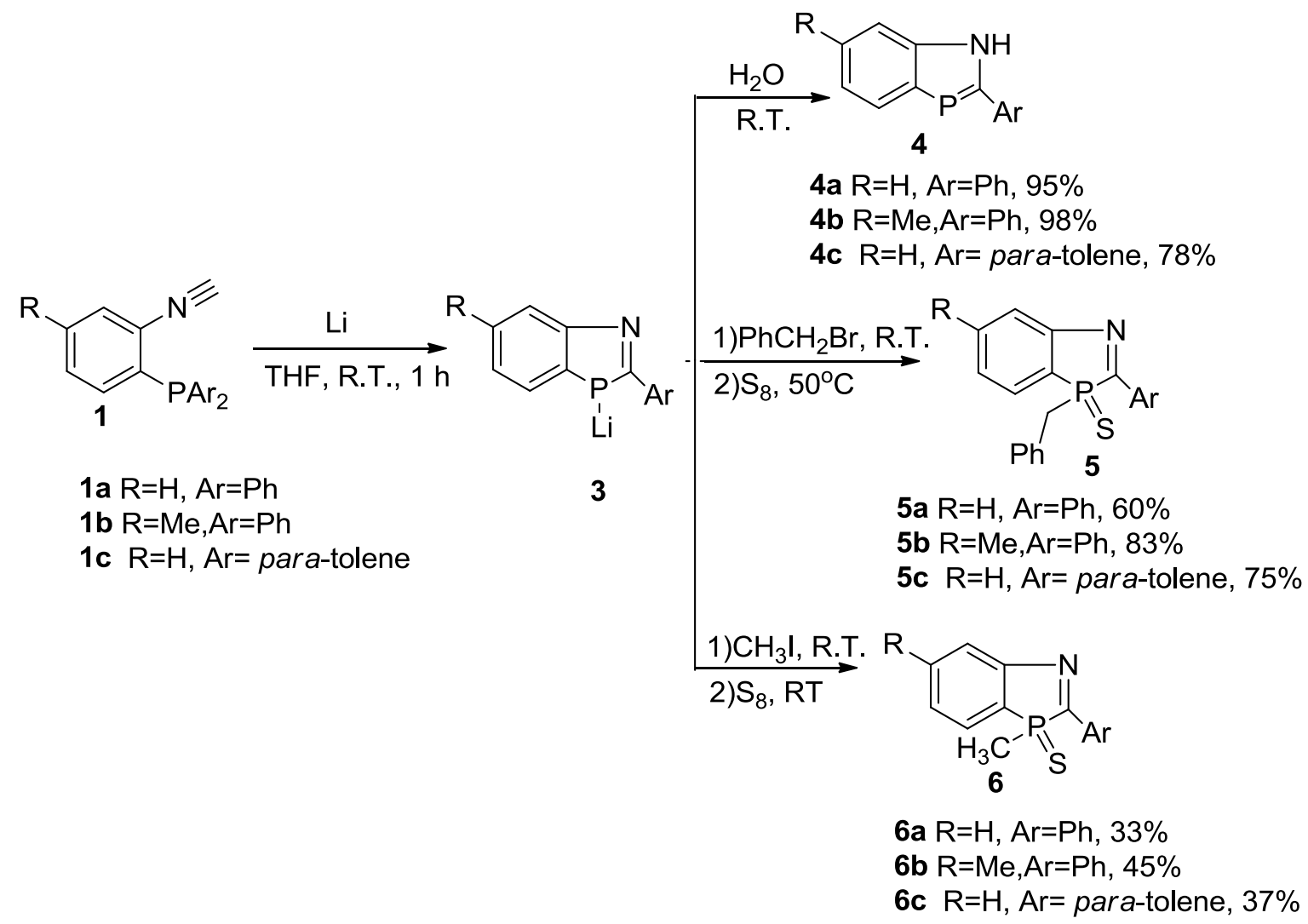

To a solution of $1(1 \mathrm{mmol})$ in THF $(10 \mathrm{~mL})$ was added 5 equivalent of 
lithium wire under $\mathrm{N}_{2}$ atmosphere. The reaction mixture was stirred for $1 \mathrm{~h}$ at room temperature and ${ }^{31} \mathrm{P}$ NMR signal indicated the reaction was complete, then the excess of lithium wire was removed. Water, benzyl bromide or iodomethane $(2 \mathrm{mmol})$ was added at room temperature. (1) For the reaction with water, the solvent was removed under reduced pressure after 10min. Anhydrous dichloromethane $(20 \mathrm{~mL})$ was added and dried over anhydrous $\mathrm{Na}_{2} \mathrm{SO}_{4}$. After stirring for $30 \mathrm{~min}$, the reaction mixture was filtrated under $\mathrm{N}_{2}$ atmosphere and removed again under reduced pressure . The products $(\mathbf{4 a}, \mathbf{4 b}, \mathbf{4 c})$ were got as yellow solids. (2) For the reaction with benzyl bromide or iodomethane, The reaction mixture was stirred for $3 \mathrm{~h}$ and ${ }^{31} \mathrm{P}$ NMR signal indicated the reaction was completed. Then $S_{8}$ was added, the reaction mixture was stirred for another $3 \mathrm{~h}$ at $50^{\circ} \mathrm{C}$. After removal of the solvent under reduced pressure, the residue was treated with water $(10 \mathrm{~mL})$, and extracted with dichloromethane. The organic layer was dried over anhydrous $\mathrm{Na}_{2} \mathrm{SO}_{4}$. After filtration and removal of the solvent, the residue was chromatographed on silica gel (petroleum ether/ethyl acetate $=15 / 1$ ).

$\mathbf{4 a}^{[2]}$ : yellow solid, 201mg, 95\% yield, purity $>95 \%$. ${ }^{31} \mathrm{P}$ NMR $(121 \mathrm{MHz}$, $\left.\mathrm{CDCl}_{3}\right): \delta=75.6 \mathrm{ppm} ;{ }^{1} \mathrm{H}$ NMR $(300 \mathrm{MHz}, \mathrm{CDCl} 3): \delta=7.16-7.22(\mathrm{~m}, 1 \mathrm{H})$, 7.34-7.48 (m, 4H), 7.60-7.63 (d, J=8.4Hz, 1H), 7.79-7.83 (m, 2H), 8.09 $(\mathrm{dq}, J=3.6,7.8 \mathrm{~Hz}, 1 \mathrm{H}), 9.56(\mathrm{brs}, 1 \mathrm{H}, \mathrm{NH}) \mathrm{ppm} ;{ }^{13} \mathrm{C} \mathrm{NMR}(75 \mathrm{MHz}$, $\left.\mathrm{CDCl}_{3}\right): \delta=113.58(\mathrm{~s}, \mathrm{CH}), 120.54\left(\mathrm{~d}, J_{\mathrm{CP}}=12.1 \mathrm{~Hz}, \mathrm{CH}\right), 125.28(\mathrm{~d}$, 
$\left.J_{\mathrm{CP}}=2.3 \mathrm{~Hz}, 2 \mathrm{CH}\right), 125.43(\mathrm{~s}, \mathrm{CH}), 128.86\left(\mathrm{~d}, J_{\mathrm{CP}}=21.1 \mathrm{~Hz}, \mathrm{CH}\right), 128.93(\mathrm{~d}$, $\left.J_{\mathrm{CP}}=3.0 \mathrm{~Hz}, \mathrm{CH}\right), 129.19(\mathrm{~s}, 2 \mathrm{CH}), 134.96\left(\mathrm{~d}, J_{\mathrm{CP}}=15.8 \mathrm{~Hz}, \mathrm{C}\right), 141.56(\mathrm{~d}$, $\left.J_{\mathrm{CP}}=41.3 \mathrm{~Hz}, \mathrm{C}\right), \quad 142.88\left(\mathrm{~d}, J_{\mathrm{CP}}=9.1 \mathrm{~Hz}, \mathrm{C}\right), \quad 174.45\left(\mathrm{~d}, J_{\mathrm{CP}}=44.5 \mathrm{~Hz}\right.$, C)ppm. HRMS calcd for $\mathrm{C}_{13} \mathrm{H}_{11} \mathrm{NP}[\mathrm{M}+\mathrm{H}]^{+}$: 212.0624; found : 212.0622 .

4b: yellow solid, 221mg, 98\% yield, purity $>95 \% .{ }^{31} \mathrm{P}$ NMR $(121 \mathrm{MHz}$, $\left.\mathrm{CDCl}_{3}\right): \delta=74.9 \mathrm{ppm} ;{ }^{1} \mathrm{H}$ NMR $\left(300 \mathrm{MHz}, \mathrm{CDCl}_{3}\right): \delta=2.50\left(\mathrm{~s}, 3 \mathrm{H}, \mathrm{CH}_{3}\right)$, 7.02(d, $J=8.1 \mathrm{~Hz}, 1 \mathrm{H}), 7.36-7.48(\mathrm{~m}, 4 \mathrm{H}), 7.79(\mathrm{~d}, J=7.5 \mathrm{~Hz}, 2 \mathrm{H}), 7.95(\mathrm{dq}$, $J=3.6,8.1 \mathrm{~Hz}, 1 \mathrm{H}), 9.33(\mathrm{brs}, 1 \mathrm{H}, \mathrm{NH}) \mathrm{ppm} ;{ }^{13} \mathrm{C} \mathrm{NMR}\left(75 \mathrm{MHz}, \mathrm{CDCl}_{3}\right)$ : $\delta=21.73\left(\mathrm{~s}, \mathrm{CH}_{3}\right), 113.41(\mathrm{~s}, \mathrm{CH}), 122.61\left(\mathrm{~d}, J_{\mathrm{CP}}=12.1 \mathrm{~Hz}, \mathrm{CH}\right), 125.17(\mathrm{~d}$, $\left.J_{\mathrm{CP}}=12.8 \mathrm{~Hz}, 2 \mathrm{CH}\right), 128.48\left(\mathrm{~d}, J_{\mathrm{CP}}=21.1 \mathrm{~Hz}, \mathrm{CH}\right), 128.75\left(\mathrm{~d}, J_{\mathrm{CP}}=3.0 \mathrm{~Hz}\right.$, $\mathrm{CH}), 129.17(\mathrm{~s}, 2 \mathrm{CH}), 135.10\left(\mathrm{~d}, J_{\mathrm{CP}}=15.1 \mathrm{~Hz}, \mathrm{C}\right), 135.54\left(\mathrm{~d}, J_{\mathrm{CP}}=3.0 \mathrm{~Hz}, \mathrm{C}\right)$, $138.22\left(\mathrm{~d}, \quad J_{\mathrm{CP}}=40.0 \mathrm{~Hz}, \quad \mathrm{C}\right), \quad 143.43\left(\mathrm{~d}, \quad J_{\mathrm{CP}}=6.8 \mathrm{~Hz}, \quad \mathrm{C}\right), \quad 173.81(\mathrm{~d}$, $\left.J_{\mathrm{CP}}=49.8 \mathrm{~Hz}, \mathrm{C}\right) \mathrm{ppm}$. HRMS calcd for $\mathrm{C}_{14} \mathrm{H}_{13} \mathrm{NP}[\mathrm{M}+\mathrm{H}]^{+}:$226.0780; found : 226.0778 .

4c: yellow solid, 176mg, 78\% yield, purity $>95 \%$. ${ }^{31} \mathrm{P}$ NMR $(121 \mathrm{MHz}$, $\left.\mathrm{CDCl}_{3}\right): \delta=73.4 \mathrm{ppm} ;{ }^{1} \mathrm{H}$ NMR $\left(300 \mathrm{MHz}, \mathrm{CDCl}_{3}\right): \delta=2.37\left(\mathrm{~s}, 3 \mathrm{H}, \mathrm{CH}_{3}\right)$, 7.11-7.23(m, 3H), 7.28-7.34(m, 1H), 7.54-7.57(m, 1H), 7.64-7.67(dd, $J=1.8,8.1 \mathrm{~Hz}, 2 \mathrm{H}), 8.03(\mathrm{dq}, J=3.6,7.8 \mathrm{~Hz}, 1 \mathrm{H}), 9.41(\mathrm{brs}, 1 \mathrm{H}, \mathrm{NH}) \mathrm{ppm}$; ${ }^{13} \mathrm{C}$ NMR $\left(75 \mathrm{MHz}, \mathrm{CDCl}_{3}\right): \delta=21.32\left(\mathrm{~s}, \mathrm{CH}_{3}\right), 113.48(\mathrm{~s}, \mathrm{CH}), 120.50(\mathrm{~d}$, $\left.J_{\mathrm{CP}}=11.3 \mathrm{~Hz}, \mathrm{CH}\right), 125.10\left(\mathrm{~d}, J_{\mathrm{CP}}=3.0 \mathrm{~Hz}, \mathrm{CH}\right), 125.20\left(\mathrm{~d}, J_{\mathrm{CP}}=12.1 \mathrm{~Hz}\right.$, $2 \mathrm{CH}), \quad 128.80\left(\mathrm{~d}, \quad J_{\mathrm{CP}}=21.1 \mathrm{~Hz}, \quad \mathrm{CH}\right), \quad 129.86(\mathrm{~s}, \quad 2 \mathrm{CH}), \quad 132.22(\mathrm{~d}$, 
$\left.J_{\mathrm{CP}}=15.8 \mathrm{~Hz}, \mathrm{C}\right), 139.10\left(\mathrm{~d}, J_{\mathrm{CP}}=3.0 \mathrm{~Hz}, \mathrm{C}\right), 141.54\left(\mathrm{~d}, J_{\mathrm{CP}}=41.5 \mathrm{~Hz}, \mathrm{C}\right)$, $142.82\left(\mathrm{~d}, J_{\mathrm{CP}}=6.8 \mathrm{~Hz}, \mathrm{C}\right), 174.81\left(\mathrm{~d}, J_{\mathrm{CP}}=50.6 \mathrm{~Hz}, \mathrm{C}\right) \mathrm{ppm}$. HRMS calcd for $\mathrm{C}_{14} \mathrm{H}_{13} \mathrm{NP}[\mathrm{M}+\mathrm{H}]^{+}:$226.0780; found : 226.0778 .

5a: m.p. $131-133{ }^{\circ} \mathrm{C}$, yellow solid, 200mg, 60\% yield. ${ }^{31} \mathrm{P}$ NMR $\left(121 \mathrm{MHz}, \quad \mathrm{CDCl}_{3}\right): \quad \delta=49.5 \mathrm{ppm} ;{ }^{1} \mathrm{H} \quad \mathrm{NMR} \quad(300 \mathrm{MHz}, \quad \mathrm{CDCl} 3)$ : $\delta=3.53-3.76(\mathrm{~m}, 2 \mathrm{H}), 6.80(\mathrm{dd}, J=1.8,7.2 \mathrm{~Hz}, 2 \mathrm{H}), 7.09(\mathrm{t}, J=7.8 \mathrm{~Hz}, 2 \mathrm{H})$, 7.14-7.19(m, 1H), 7.38-7.43(m, 1H), 7.48-7.61(m, 6H), 8.43(dq, $J=0.6$, $7.2 \mathrm{~Hz}, 2 \mathrm{H}) \mathrm{ppm} ;{ }^{13} \mathrm{C} \mathrm{NMR}\left(75 \mathrm{MHz}, \mathrm{CDCl}_{3}\right): \delta=42.58\left(\mathrm{~d}, J_{\mathrm{CP}}=39.2 \mathrm{~Hz}\right.$, $\left.\mathrm{CH}_{2}\right), 124.70\left(\mathrm{~d}, J_{\mathrm{CP}}=5.0 \mathrm{~Hz}, \mathrm{CH}\right), 126.02(\mathrm{~s}, \mathrm{C}), 127.66\left(\mathrm{~d}, J_{\mathrm{CP}}=4.0 \mathrm{~Hz}, \mathrm{CH}\right)$, $128.23\left(\mathrm{~d}, J_{\mathrm{CP}}=3.5 \mathrm{~Hz}, 2 \mathrm{CH}\right), 128.61(\mathrm{~s}, \mathrm{CH}), 128.74(\mathrm{~s}, \mathrm{CH}), 128.89(\mathrm{~d}$, $\left.J_{\mathrm{CP}}=3.4 \mathrm{~Hz}, 2 \mathrm{CH}\right), 129.15(\mathrm{~s}, 2 \mathrm{CH}), 129.44\left(\mathrm{~d}, J_{\mathrm{CP}}=9.0 \mathrm{~Hz}, \mathrm{C}\right), 129.77(\mathrm{~d}$, $\left.J_{\mathrm{CP}}=5.5 \mathrm{~Hz}, 2 \mathrm{CH}\right), 132.54(\mathrm{~s}, \mathrm{CH}), 133.08\left(\mathrm{~d}, J_{\mathrm{CP}}=26.2 \mathrm{~Hz}, \mathrm{C}\right), 133.97(\mathrm{~d}$, $\left.J_{\mathrm{CP}}=2.0 \mathrm{~Hz}, \mathrm{CH}\right), 153.41\left(\mathrm{~d}, J_{\mathrm{CP}}=31.7 \mathrm{~Hz}, \mathrm{C}\right), 170.08\left(\mathrm{~d}, J_{\mathrm{CP}}=42.3 \mathrm{~Hz}, \mathrm{C}\right)$ ppm. HRMS calcd for $\mathrm{C}_{20} \mathrm{H}_{17} \mathrm{NPS}[\mathrm{M}+\mathrm{H}]^{+}$: 334.0814; found : 334.0817 . Anal. Calcd for $\mathrm{C}_{20} \mathrm{H}_{16} \mathrm{NPS}$ : C, 72.05; H, 4.84; N, 4.20; S, 9.62. found: $\mathrm{C}$, 71.85; H, 4.82; N, 4.08; S, 9.45.

5b: m.p. $127-129{ }^{\circ} \mathrm{C}$, yellow solid, $288 \mathrm{mg}, 83 \%$ yield. ${ }^{31} \mathrm{P}$ NMR $\left(121 \mathrm{MHz}, \mathrm{CDCl}_{3}\right): \delta=49.0 \mathrm{ppm} ;{ }^{1} \mathrm{H} \mathrm{NMR}\left(300 \mathrm{MHz}, \mathrm{CDCl}_{3}\right): \delta=2.43(\mathrm{~s}$, $\left.3 \mathrm{H}, \mathrm{CH}_{3}\right), 3.50-3.75\left(\mathrm{~m}, 2 \mathrm{H}, \mathrm{CH}_{2}\right), 6.81-6.83(\mathrm{~m}, 2 \mathrm{H}), 7.07-7.24(\mathrm{~m}, 4 \mathrm{H})$, 7.34-7.59(m, 5H), 8.44(d, J=6.9Hz, 2H)ppm; $\left.{ }^{13} \mathrm{C} \mathrm{NMR} \mathrm{(75MHz,} \mathrm{CDCl}_{3}\right)$ : $\delta=21.93\left(\mathrm{~s}, \mathrm{CH}_{3}\right), 42.70\left(\mathrm{~d}, J_{\mathrm{CP}}=40.0 \mathrm{~Hz}, \mathrm{CH}_{2}\right), 122.07\left(\mathrm{~d}, J_{\mathrm{CP}}=99.6 \mathrm{~Hz}, \mathrm{C}\right)$, $125.47\left(\mathrm{~d}, \quad J_{\mathrm{CP}}=5.3 \mathrm{~Hz}, \quad \mathrm{CH}\right), \quad 127.60\left(\mathrm{~d}, J_{\mathrm{CP}}=3.8 \mathrm{~Hz}, \mathrm{CH}\right), \quad 128.21(\mathrm{~d}$, 
$\left.J_{\mathrm{CP}}=3.8 \mathrm{~Hz}, 2 \mathrm{CH}\right), 128.59\left(\mathrm{~d}, J_{\mathrm{CP}}=12.1 \mathrm{~Hz}, \mathrm{CH}\right), 128.83\left(\mathrm{~d}, J_{\mathrm{CP}}=3.0 \mathrm{~Hz}\right.$, $2 \mathrm{CH}), 129.09(\mathrm{~s}, 2 \mathrm{CH}), 129.40\left(\mathrm{~d}, J_{\mathrm{CP}}=10.6 \mathrm{~Hz}, \mathrm{CH}\right), 129.61\left(\mathrm{~d}, J_{\mathrm{CP}}=9.1 \mathrm{~Hz}\right.$, C), $129.78\left(\mathrm{~d}, J_{\mathrm{CP}}=5.3 \mathrm{~Hz}, 2 \mathrm{CH}\right), 132.40(\mathrm{~s}, \mathrm{CH}), 133.18\left(\mathrm{~d}, J_{\mathrm{CP}}=25.7 \mathrm{~Hz}, \mathrm{C}\right)$, $144.89\left(\mathrm{~d}, \quad J_{\mathrm{CP}}=2.1 \mathrm{~Hz}, \quad \mathrm{C}\right), \quad 153.82\left(\mathrm{~d}, \quad J_{\mathrm{CP}}=32.5 \mathrm{~Hz}, \quad \mathrm{C}\right), \quad 170.41(\mathrm{~d}$, $\left.J_{\mathrm{CP}}=42.3 \mathrm{~Hz}, \mathrm{C}\right)$ ppm. HRMS calcd for $\mathrm{C}_{21} \mathrm{H}_{19} \mathrm{NPS}[\mathrm{M}+\mathrm{H}]^{+}: 348.0970$; found : 348.0978. Anal. Calcd for $\mathrm{C}_{21} \mathrm{H}_{18} \mathrm{NPS}$ : C, 72.60; H, 5.22; N, 4.03; S, 9.23. found: C, 72.49; H, 5.17; N, 4.05; S, 9.09.

5c: m.p. $120-122{ }^{\circ} \mathrm{C}$, yellow solid, $261 \mathrm{mg}, 75 \%$ yield. ${ }^{31} \mathrm{P}$ NMR (121MHz, CDCl3): $\delta=49.6 \mathrm{ppm} ;{ }^{1} \mathrm{H}$ NMR $\left(300 \mathrm{MHz}, \mathrm{CDCl}_{3}\right): \delta=2.47(\mathrm{~s}$, $\left.3 \mathrm{H}, \mathrm{CH}_{3}\right), 3.51-3.74\left(\mathrm{~m}, 2 \mathrm{H}, \mathrm{CH}_{2}\right), 6.77-6.81(\mathrm{~m}, 2 \mathrm{H}), 7.06-7.19(\mathrm{~m}, 3 \mathrm{H})$, 7.32-7.40 (m, 3H), $7.48(\mathrm{t}, J=7.8 \mathrm{~Hz}, 1 \mathrm{H}), 7.53-7.60(\mathrm{~m}, 2 \mathrm{H}), 8.32(\mathrm{~d}$, $J=8.1 \mathrm{~Hz}, 2 \mathrm{H}) \mathrm{ppm} ;{ }^{13} \mathrm{C} \mathrm{NMR}\left(75 \mathrm{MHz}, \mathrm{CDCl}_{3}\right): \delta=21.85\left(\mathrm{~s}, \mathrm{CH}_{3}\right), 42.74(\mathrm{~d}$, $\left.J_{\mathrm{CP}}=39.2 \mathrm{~Hz}, \mathrm{CH}_{2}\right), 124.41\left(\mathrm{~d}, J_{\mathrm{CP}}=5.1 \mathrm{~Hz}, \mathrm{CH}\right), 125.19\left(\mathrm{~d}, J_{\mathrm{CP}}=97.1 \mathrm{~Hz}, \mathrm{C}\right)$, $127.57\left(\mathrm{~d}, \quad J_{\mathrm{CP}}=4.1 \mathrm{~Hz}, \mathrm{CH}\right), \quad 128.15\left(\mathrm{~d}, J_{\mathrm{CP}}=3.5 \mathrm{~Hz}, 2 \mathrm{CH}\right), \quad 128.31(\mathrm{~d}$, $\left.J_{\mathrm{CP}}=10.3 \mathrm{~Hz}, \mathrm{CH}\right), 128.66(\mathrm{~s}, \mathrm{CH}), 128.85\left(\mathrm{~d}, J_{\mathrm{CP}}=3.8 \mathrm{~Hz}, 2 \mathrm{CH}\right), 129.52(\mathrm{~d}$, $\left.J_{\mathrm{CP}}=9.0 \mathrm{~Hz}, \mathrm{C}\right), 129.70\left(\mathrm{~d}, J_{\mathrm{CP}}=5.5 \mathrm{~Hz}, 2 \mathrm{CH}\right), 129.86(\mathrm{~s}, 2 \mathrm{CH}), 130.41(\mathrm{~d}$, $\left.J_{\mathrm{CP}}=26.4 \mathrm{~Hz}, \mathrm{C}\right), 133.83\left(\mathrm{~d}, J_{\mathrm{CP}}=2.3 \mathrm{~Hz}, \mathrm{CH}\right), 143.32(\mathrm{~s}, \mathrm{C}), 153.61(\mathrm{~d}$, $\left.J_{\mathrm{CP}}=32.5 \mathrm{~Hz}, \mathrm{C}\right), 170.00\left(\mathrm{~d}, J_{\mathrm{CP}}=41.5 \mathrm{~Hz}, \mathrm{C}\right) \mathrm{ppm}$. HRMS calcd for $\mathrm{C}_{21} \mathrm{H}_{19} \mathrm{NPS}[\mathrm{M}+\mathrm{H}]^{+}:$348.0970; found : 348.0977. Anal. Calcd for $\mathrm{C}_{21} \mathrm{H}_{18} \mathrm{NPS}$ : C, 72.60; H, 5.22; N, 4.03; S, 9.23. found: C, 72.85; H, 5.32; N, 3.93; S, 8.85.

6a: m.p. $108-110{ }^{\circ} \mathrm{C}$, yellow solid, $85 \mathrm{mg}, 33 \%$ yield. ${ }^{31} \mathrm{P}$ NMR $(121 \mathrm{MHz}$, 
$\mathrm{CDCl} 3): \delta=42.7 \mathrm{ppm} ;{ }^{1} \mathrm{H} \mathrm{NMR}\left(300 \mathrm{MHz}, \mathrm{CDCl}_{3}\right): \delta=2.06\left(\mathrm{~d}, J_{\mathrm{HP}}=13.2 \mathrm{~Hz}\right.$, $\left.3 \mathrm{H}, \mathrm{CH}_{3}\right), 7.43-7.50(\mathrm{~m}, 1 \mathrm{H}), 7.52-7.59(\mathrm{~m}, 3 \mathrm{H}), 7.61-7.67(\mathrm{~m}, 1 \mathrm{H})$, 7.75-7.83(m, $2 \mathrm{H}), \quad 8.45-8.48(\mathrm{~m}, \quad 2 \mathrm{H}) \mathrm{ppm} ; \quad{ }^{13} \mathrm{C} \quad \mathrm{NMR}(75 \mathrm{MHz}$, $\left.\mathrm{CDCl}_{3}\right): \delta=21.33\left(\mathrm{~d}, \quad J_{\mathrm{CP}}=48.3 \mathrm{~Hz}, \quad \mathrm{CH}_{3}\right), \quad 124.90\left(\mathrm{~d}, \quad J_{\mathrm{CP}}=5.1 \mathrm{~Hz}, \quad \mathrm{CH}\right)$, $127.46\left(\mathrm{~d}, \quad J_{\mathrm{CP}}=98.1 \mathrm{~Hz}, \mathrm{C}\right), \quad 127.77\left(\mathrm{~d}, \quad J_{\mathrm{CP}}=12.8 \mathrm{~Hz}, \mathrm{CH}\right), \quad 128.78(\mathrm{~d}$, $\left.J_{\mathrm{CP}}=3.8 \mathrm{~Hz}, 2 \mathrm{CH}\right), 129.04\left(\mathrm{~d}, J_{\mathrm{CP}}=10.5 \mathrm{~Hz}, \mathrm{CH}\right), 129.16(\mathrm{~s}, 2 \mathrm{CH}), 132.27(\mathrm{~d}$, $\left.J_{\mathrm{CP}}=27.2 \mathrm{~Hz}, \mathrm{C}\right), 132.55(\mathrm{~s}, \mathrm{CH}), 133.85\left(\mathrm{~d}, J_{\mathrm{CP}}=2.0 \mathrm{~Hz}, \mathrm{CH}\right), 152.90(\mathrm{~d}$, $\left.J_{\mathrm{CP}}=34.0 \mathrm{~Hz}, \mathrm{C}\right), 171.11\left(\mathrm{~d}, J_{\mathrm{CP}}=45.3 \mathrm{~Hz}, \mathrm{C}\right) \mathrm{ppm}$. HRMS calcd for $\mathrm{C}_{14} \mathrm{H}_{13} \mathrm{NPS}[\mathrm{M}+\mathrm{H}]^{+}:$258.0501; found : 258.0508. Anal. Calcd for $\mathrm{C}_{14} \mathrm{H}_{12} \mathrm{NPS}$ : C, 65.35; H, 4.70; N, 5.44; S, 12.46. found: C, 65.22; H, 4.79; $\mathrm{N}, 5.34 ; \mathrm{S}, 12.57$.

6b: m.p. $123-125{ }^{\circ} \mathrm{C}$, yellow solid, $122 \mathrm{mg}$, $45 \%$ yield. ${ }^{31} \mathrm{P}$ NMR $\left(121 \mathrm{MHz}, \mathrm{CDCl}_{3}\right): \delta=42.2 \mathrm{ppm} ;{ }^{1} \mathrm{H} \mathrm{NMR}\left(300 \mathrm{MHz}, \mathrm{CDCl}_{3}\right): \delta=2.03(\mathrm{~d}$, $\left.J_{\mathrm{HP}}=13.2 \mathrm{~Hz}, 3 \mathrm{H}, \mathrm{CH}_{3}\right), 2.48\left(\mathrm{~s}, 3 \mathrm{H}, \mathrm{CH}_{3}\right), 7.26-7.29(\mathrm{~m}, 1 \mathrm{H}), 7.53-7.59(\mathrm{~m}$, $4 \mathrm{H}), 7.67(\mathrm{t}, J=8.1 \mathrm{~Hz}, 1 \mathrm{H}), 8.44-8.47(\mathrm{~m}, 2 \mathrm{H}) \mathrm{ppm} ;{ }^{13} \mathrm{C} \mathrm{NMR}(75 \mathrm{MHz}$, $\left.\mathrm{CDCl}_{3}\right): \delta=21.52\left(\mathrm{~d}, \quad J_{\mathrm{CP}}=48.3 \mathrm{~Hz}, \quad \mathrm{CH}_{3}\right), \quad 21.87\left(\mathrm{~s}, \quad \mathrm{CH}_{3}\right), \quad 124.15(\mathrm{~d}$, $\left.J_{\mathrm{CP}}=100.4 \mathrm{~Hz}, \mathrm{C}\right), 125.65\left(\mathrm{~d}, J_{\mathrm{CP}}=5.3 \mathrm{~Hz}, \mathrm{CH}\right), 127.55\left(\mathrm{~d}, J_{\mathrm{CP}}=13.6 \mathrm{~Hz}, \mathrm{CH}\right)$, $128.74\left(\mathrm{~d}, J_{\mathrm{CP}}=3.8 \mathrm{~Hz}, 2 \mathrm{CH}\right), 129.12(\mathrm{~s}, 2 \mathrm{CH}), 129.66\left(\mathrm{~d}, J_{\mathrm{CP}}=10.6 \mathrm{~Hz}, \mathrm{CH}\right)$, $132.36\left(\mathrm{~d}, J_{\mathrm{CP}}=27.2 \mathrm{~Hz}, \mathrm{C}\right), 132.44(\mathrm{~s}, \mathrm{CH}), 144.91\left(\mathrm{~d}, J_{\mathrm{CP}}=3.0 \mathrm{~Hz}, \mathrm{C}\right)$, $153.30\left(\mathrm{~d}, J_{\mathrm{CP}}=34.0 \mathrm{~Hz}, \mathrm{C}\right), 171.38\left(\mathrm{~d}, J_{\mathrm{CP}}=47.5 \mathrm{~Hz}, \mathrm{C}\right) \mathrm{ppm}$. HRMS calcd for $\mathrm{C}_{15} \mathrm{H}_{15} \mathrm{NPS}[\mathrm{M}+\mathrm{H}]^{+}:$272.0657; found : 272.0665. Anal. Calcd for $\mathrm{C}_{15} \mathrm{H}_{14} \mathrm{NPS}$ : C, 66.40; H, 5.20; N, 5.16; S, 11.82. found: C, 66.21; H, 5.31; 
N, 5.04; S, 11.66.

6c: m.p. 101-102 ${ }^{\circ} \mathrm{C}$, yellow solid, 100mg, 37\% yield. ${ }^{31} \mathrm{P}$ NMR (121MHz, CDCl3): $\delta=42.5 \mathrm{ppm} ;{ }^{1} \mathrm{H}$ NMR $\left(300 \mathrm{MHz}, \mathrm{CDCl}_{3}\right): \delta=2.04(\mathrm{~d}$, $\left.J_{\mathrm{HP}}=8.4 \mathrm{~Hz}, 3 \mathrm{H}\right), 2.45(\mathrm{~s}, 3 \mathrm{H}), 7.35(\mathrm{~d}, J=8.4 \mathrm{~Hz}, 2 \mathrm{H}), 7.44(\mathrm{ddd}, J=0.9,3.9$, $7.5 \mathrm{~Hz}, 1 \mathrm{H}), 7.59-7.65(\mathrm{~m}, 1 \mathrm{H}), 7.72-7.81(\mathrm{~m}, 2 \mathrm{H}), 8.35(\mathrm{~d}, J=8.4 \mathrm{~Hz}, 2 \mathrm{H})$ ppm; ${ }^{13} \mathrm{C}$ NMR $\left(75 \mathrm{MHz}, \mathrm{CDCl}_{3}\right): \delta=21.42\left(\mathrm{~d}, J_{\mathrm{CP}}=47.9 \mathrm{~Hz}, \mathrm{CH}_{3}\right), 21.86(\mathrm{~s}$, $\left.\mathrm{CH}_{3}\right), 124.69\left(\mathrm{~d}, J_{\mathrm{CP}}=5.1 \mathrm{~Hz}, \mathrm{CH}\right), 127.32\left(\mathrm{~d}, J_{\mathrm{CP}}=98.4 \mathrm{~Hz}, \mathrm{C}\right), 127.73(\mathrm{~d}$, $\left.J_{\mathrm{CP}}=12.8 \mathrm{~Hz}, \mathrm{CH}\right), 128.73\left(\mathrm{~d}, J_{\mathrm{CP}}=9.8 \mathrm{~Hz}, \mathrm{CH}\right), 128.80\left(\mathrm{~d}, J_{\mathrm{CP}}=3.8 \mathrm{~Hz}, 2 \mathrm{CH}\right)$, $129.62\left(\mathrm{~d}, J_{\mathrm{CP}}=26.9 \mathrm{~Hz}, \mathrm{C}\right), 129.95(\mathrm{~s}, 2 \mathrm{CH}), 133.89\left(\mathrm{~d}, J_{\mathrm{CP}}=2.1 \mathrm{~Hz}, \mathrm{CH}\right)$, $143.47(\mathrm{~s}, \mathrm{C}), 153.06\left(\mathrm{~d}, J_{\mathrm{CP}}=33.2 \mathrm{~Hz}, \mathrm{C}\right), 170.99\left(\mathrm{~d}, J_{\mathrm{CP}}=45.3 \mathrm{~Hz}, \mathrm{C}\right) \mathrm{ppm}$. HRMS calcd for $\mathrm{C}_{15} \mathrm{H}_{15} \mathrm{NPS}[\mathrm{M}+\mathrm{H}]^{+}:$272.0657; found : 272.0664. Anal. Calcd for $\mathrm{C}_{15} \mathrm{H}_{14} \mathrm{NPS}$ : C, 66.40; H, 5.20; N, 5.16; S, 11.82. found: $\mathrm{C}$, 66.19; H, 5.18; N, 5.04; S, 11.86.

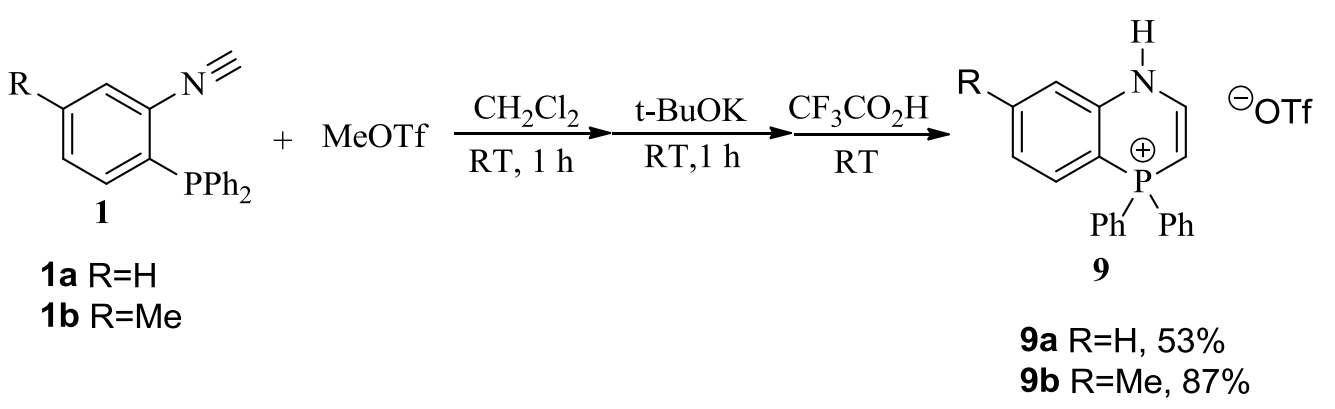

To a solution of $\mathbf{1}(1 \mathrm{mmol})$ in anhydrous dichloromethane $(10 \mathrm{~mL})$ was added MeOTf $(1.1 \mathrm{mmol})$ under $\mathrm{N}_{2}$ atmosphere. The reaction mixture was stirred for $1 \mathrm{~h}$ at room temperature and the ${ }^{31} \mathrm{P}$ NMR spectrum indicated the reaction was complete, then $t$-BuOk $(2 \mathrm{mmol})$ was added. The ${ }^{31} \mathrm{P}$ NMR spectrum showed that the reaction was complete after another $1 \mathrm{~h}$. 
Then TFA (2.1mmol) was added. After filtration, the solvent was removed under reduced pressure. The crude product was purified by column chromatography on silica gel $\left(\mathrm{CH}_{2} \mathrm{Cl}_{2} / \mathrm{CH}_{3} \mathrm{OH}=10: 1\right)$.

9a: yellow oil, $239 \mathrm{mg}, 53 \%$ yield. ${ }^{31} \mathrm{P} \mathrm{NMR}\left(121 \mathrm{MHz}, \mathrm{CDCl}_{3}\right): \delta$ $=-3.8 \mathrm{ppm} ;{ }^{19} \mathrm{~F} \mathrm{NMR}\left(\mathrm{CDCl}_{3}\right): \delta=-79.0 \mathrm{ppm} ;{ }^{1} \mathrm{H} \mathrm{NMR}\left(300 \mathrm{MHz}, \mathrm{CDCl}_{3}\right)$ : $\delta=4.84(\mathrm{dd}, J=9.9,13.2 \mathrm{~Hz}, 1 \mathrm{H}), 7.21-7.27(\mathrm{~m}, 1 \mathrm{H}), 7.46(\mathrm{ddd}, J=1.5,7.8$, 13.2Hz, $1 \mathrm{H}), 7.52-7.63(\mathrm{~m}, 9 \mathrm{H}), 7.65-7.72(\mathrm{~m}, 2 \mathrm{H}), 7.80-7.85(\mathrm{~m}, 1 \mathrm{H})$, 7.99 (ddd, $J=4.8,9.6,31.5 \mathrm{~Hz}, 1 \mathrm{H}), 12.94(\mathrm{brs}, 1 \mathrm{H}, \mathrm{NH}) \mathrm{ppm} ;{ }^{13} \mathrm{C} \mathrm{NMR}$ $\left(75 \mathrm{MHz}, \mathrm{CDCl}_{3}\right): \delta=66.51\left(\mathrm{~d}, J_{\mathrm{CP}}=95.8 \mathrm{~Hz}, \mathrm{CH}\right), 97.31\left(\mathrm{~d}, J_{\mathrm{CP}}=90.6 \mathrm{~Hz}, \mathrm{C}\right)$, $120.49\left(\mathrm{~d}, \quad J_{\mathrm{CP}}=6.0 \mathrm{~Hz}, \mathrm{CH}\right), \quad 124.78\left(\mathrm{~d}, J_{\mathrm{CP}}=11.8 \mathrm{~Hz}, \mathrm{CH}\right), \quad 125.39(\mathrm{~d}$, $\left.J_{\mathrm{CP}}=94.5 \mathrm{~Hz}, 2 \mathrm{C}\right), 129.92\left(\mathrm{~d}, J_{\mathrm{CP}}=12.8 \mathrm{~Hz}, 4 \mathrm{CH}\right), 131.00\left(\mathrm{~d}, J_{\mathrm{CP}}=6.8 \mathrm{~Hz}\right.$, $\mathrm{CH}), 132.56\left(\mathrm{~d}, J_{\mathrm{CP}}=12.1 \mathrm{~Hz}, 4 \mathrm{CH}\right), 134.09\left(\mathrm{~d}, J_{\mathrm{CP}}=3.0 \mathrm{~Hz}, 2 \mathrm{CH}\right), 134.74(\mathrm{~d}$, $\left.J_{\mathrm{CP}}=1.5 \mathrm{~Hz}, \mathrm{CH}\right), 142.50\left(\mathrm{~d}, J_{\mathrm{CP}}=3.0 \mathrm{~Hz}, \mathrm{C}\right), 145.99\left(\mathrm{~d}, J_{\mathrm{CP}}=2.3 \mathrm{~Hz}, \mathrm{CH}\right) \mathrm{ppm}$. HRMS calcd for $\mathrm{C}_{20} \mathrm{H}_{17} \mathrm{NP}$ [M- OTf] ${ }^{+}$: 302.1093; found : 302.1094 .

9b: yellow oil, 405mg, $87 \%$ yield. ${ }^{31} \mathrm{P}$ NMR (121MHz, $\left.\mathrm{CDCl}_{3}\right)$ : $\delta=-4.1 \mathrm{ppm} ;{ }^{19} \mathrm{~F}$ NMR $\left(\mathrm{CDCl}_{3}\right): \delta=-78.2 \mathrm{ppm} ;{ }^{1} \mathrm{H} \mathrm{NMR}\left(300 \mathrm{MHz}, \mathrm{CDCl}_{3}\right)$ : $\delta=2.33\left(\mathrm{~s}, 3 \mathrm{H}, \mathrm{CH}_{3}\right), 4.80(\mathrm{dd}, J=9.6,13.2 \mathrm{~Hz}, 1 \mathrm{H}), 7.05(\mathrm{~d}, J=8.1 \mathrm{~Hz}, 1 \mathrm{H})$, 7.34(dd, $J=8.1,13.2 \mathrm{~Hz}, 1 \mathrm{H}), 7.49-7.66(\mathrm{~m}, 11 \mathrm{H}), 7.94(\mathrm{ddd}, J=6.6,16.5$, $31.5 \mathrm{~Hz}, \quad 1 \mathrm{H}), \quad 12.72(\mathrm{brs}, \quad 1 \mathrm{H}, \quad \mathrm{NH}) \mathrm{ppm} ;{ }^{13} \mathrm{C} \quad \mathrm{NMR} \quad(75 \mathrm{MHz}$, $\left.\mathrm{CDCl}_{3}\right): \delta=21.69\left(\mathrm{~s}, \mathrm{CH}_{3}\right), 66.54\left(\mathrm{~d}, J_{\mathrm{CP}}=96.6 \mathrm{~Hz}, \mathrm{CH}\right), 94.34\left(\mathrm{~d}, J_{\mathrm{CP}}=92.8 \mathrm{~Hz}\right.$, C), $119.99\left(\mathrm{~d}, J_{\mathrm{CP}}=6.8 \mathrm{~Hz}, \mathrm{CH}\right), 125.59\left(\mathrm{~d}, J_{\mathrm{CP}}=94.3 \mathrm{~Hz}, 2 \mathrm{C}\right), 126.46(\mathrm{~d}$, $\left.J_{\mathrm{CP}}=12.1 \mathrm{~Hz}, \mathrm{CH}\right), 129.84\left(\mathrm{~d}, J_{\mathrm{CP}}=13.6 \mathrm{~Hz}, 4 \mathrm{CH}\right), 130.88\left(\mathrm{~d}, J_{\mathrm{CP}}=6.8 \mathrm{~Hz}\right.$, 
$\mathrm{CH}), 132.45\left(\mathrm{~d}, J_{\mathrm{CP}}=11.3 \mathrm{~Hz}, 4 \mathrm{CH}\right), 133.96\left(\mathrm{~d}, J_{\mathrm{CP}}=3.0 \mathrm{~Hz}, 2 \mathrm{CH}\right), 142.49(\mathrm{~d}$, $\left.J_{\mathrm{CP}}=3.8 \mathrm{~Hz}, \mathrm{C}\right), 145.93\left(\mathrm{~d}, J_{\mathrm{CP}}=2.3 \mathrm{~Hz}, \mathrm{CH}\right), 146.17\left(\mathrm{~d}, J_{\mathrm{CP}}=1.5 \mathrm{~Hz}, \mathrm{C}\right) \mathrm{ppm}$. HRMS calcd for $\mathrm{C}_{21} \mathrm{H}_{19} \mathrm{NP}\left[\mathrm{M}\right.$ - OTf] ${ }^{+}: 316.1250$; found : 316.1253 .

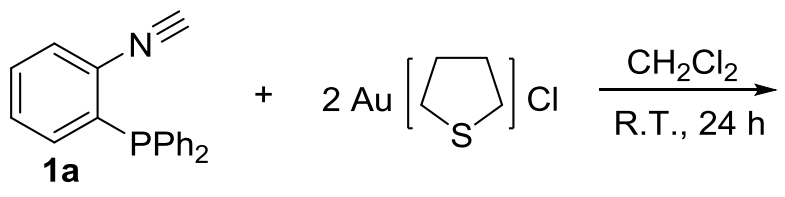

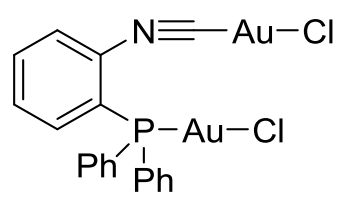

10a

Gold chloride complex (600 mg, $1.87 \mathrm{mmol})$, kept in an oven-dried 50 mL-Schlenk flask under an atmosphere of dry nitrogen, was added to a solution of 1a (253 mg, $0.89 \mathrm{mmol})$ in anhydrous dichloromethane (20 $\mathrm{mL})$. The reaction was complete after $24 \mathrm{~h}$ at room temperature. The solvent was removed under reduced pressure and washed with hexane and the mixture of dichloromethane and hexane, to give a white solid 10a(563mg, 85\% yield).

10a: decomp. $175{ }^{\circ} \mathrm{C} .{ }^{31} \mathrm{P}$ NMR $\left(121 \mathrm{MHz}, \mathrm{CDCl}_{3}\right): \delta=27.8 \mathrm{ppm} ;{ }^{1} \mathrm{H}$ NMR $\left(300 \mathrm{MHz}, \mathrm{CDCl}_{3}\right): \delta=7.10-7.17(\mathrm{~m}, 1 \mathrm{H}), 7.59-7.73(\mathrm{~m}, 13 \mathrm{H}) \mathrm{ppm}$. Anal. Calcd for $\mathrm{C}_{19} \mathrm{H}_{14} \mathrm{Au}_{2} \mathrm{Cl}_{2} \mathrm{NP}$ : C, 30.34; H, 1.88; N, 1.86. found: C, $29.75 ; \mathrm{H}, 1.81 ; \mathrm{N}, 1.87$.

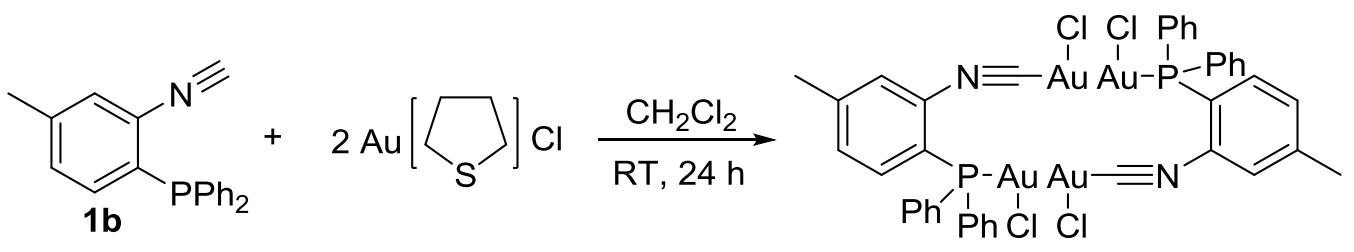
10b

10b (a colourless solid, 398mg, 52\% yield) was obtained from $\mathbf{1 b}(303 \mathrm{mg}$, $1 \mathrm{mmol}$ ) following method of the product $\mathbf{1 0 a}$.

10b: decomp. $180{ }^{\circ} \mathrm{C} .{ }^{31} \mathrm{P}$ NMR $\left(121 \mathrm{MHz}, \mathrm{CDCl}_{3}\right): \delta=27.8 \mathrm{ppm} ;{ }^{1} \mathrm{H} \mathrm{NMR}$ 
$\left(300 \mathrm{MHz}, \mathrm{CDCl}_{3}\right): \delta=2.50\left(\mathrm{~s}, 6 \mathrm{H}, \mathrm{CH}_{3}\right), 7.03(\mathrm{dd}, J=8.1,12 \mathrm{~Hz}, 2 \mathrm{H}), 7.41(\mathrm{~d}$, $J=8.1 \mathrm{~Hz}, 2 \mathrm{H}), 7.55-7.70(\mathrm{~m}, 22 \mathrm{H}) \mathrm{ppm}$. Anal. Calcd for $\mathrm{C}_{40} \mathrm{H}_{32} \mathrm{Au}_{4} \mathrm{Cl}_{4} \mathrm{~N}_{2} \mathrm{P}_{2}$ : C, 31.35; H, 2.10; N, 1.83. found: C, 30.76; H, 2.03; $\mathrm{N}, 1.53$.

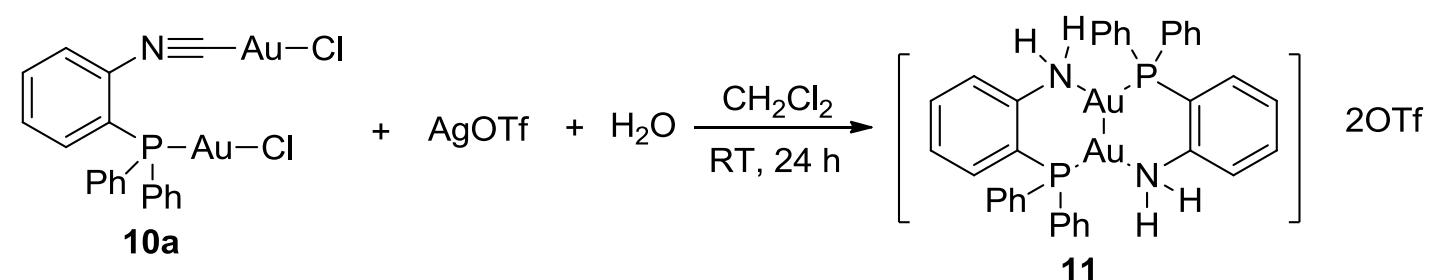
$\operatorname{AgOTf}(93 \mathrm{mg}, 0.36 \mathrm{mmol})$ was added to a solution of $\mathbf{1 0 a}(135 \mathrm{mg}$, $0.18 \mathrm{mmol})$ in anhydrous dichloromethane $(6 \mathrm{~mL}), \mathrm{kept}$ in an oven-dried $10 \mathrm{~mL}-$ Schlenk flask under an atmosphere of dry nitrogen. After $12 \mathrm{~h}, 5$ equivalents of water were added. The reaction was complete after $12 \mathrm{~h}$ at room temperature. After filtration, the solvent was removed under reduced pressure and washed with hexane and the mixture of dichloromethane and hexane, to give a colorless solid 11 (70 mg, 63\% yield).

11: decomp. $173{ }^{\circ} \mathrm{C} .{ }^{31} \mathrm{P}$ NMR $\left(121 \mathrm{MHz}, \mathrm{CDCl}_{3}\right): \delta=19.3 \mathrm{ppm} ;{ }^{19} \mathrm{~F}$ $\operatorname{NMR}\left(\mathrm{CDCl}_{3}\right) \delta=-78.6 \mathrm{ppm} ;{ }^{1} \mathrm{H}$ NMR $\left(300 \mathrm{MHz}, \mathrm{CDCl}_{3}\right): \delta=6.69-6.87(\mathrm{~m}$, 6H), 7.27-7.32(m, 2H), 7.64-7.71(m, 22H), 7.87-7.91(m, 2H)ppm. Anal. Calcd for $\mathrm{C}_{38} \mathrm{H}_{32} \mathrm{Au}_{2} \mathrm{~F}_{6} \mathrm{~N}_{2} \mathrm{O}_{6} \mathrm{P}_{2} \mathrm{~S}_{2}$ : C, 36.61; H, 2.59; N, 2.25; S, 5.14. found: $\mathrm{C}, 36.89 ; \mathrm{H}, 2.84 ; \mathrm{N}, 2.25 ; \mathrm{S}, 4.94$. 
2

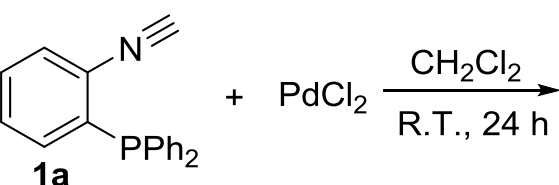<smiles>Cl[P-]1(c2ccccc2)C(Nc2ccccc2)=[NH+]c2ccccc2P1c1ccccc1</smiles>

12

$\mathrm{PdCl}_{2}(172 \mathrm{mg}, 0.97 \mathrm{mmol})$ was added to a solution of $\mathbf{1 a}(278 \mathrm{mg}, 0.97$ mmol) in anhydrous dichloromethane $(10 \mathrm{~mL})$, kept in an oven-dried 50 $\mathrm{mL}$ Schlenk flask under an atmosphere of dry nitrogen. The ${ }^{31} \mathrm{P}$ NMR spectrum of the solution indicated that the reaction was complete after 24 $\mathrm{h}$ at r.t. After filtration, the solvent was removed under reduced pressure and washed with hexane and the mixture of dichloromethane and hexane, to give a yellow solid (m.p. $203-205^{\circ} \mathrm{C}, 275 \mathrm{mg}, 76 \%$ ).

12: ${ }^{31} \mathrm{P}$ NMR $\left(121 \mathrm{MHz}, \mathrm{CDCl}_{3}\right): \delta=14.1 \mathrm{ppm} ;{ }^{1} \mathrm{H}$ NMR $(300 \mathrm{MHz}$, $\left.\mathrm{CDCl}_{3}\right): \delta=7.05-7.10(\mathrm{~m}, 2 \mathrm{H}), 7.21-7.27(\mathrm{~m}, 3 \mathrm{H}), 7.39-7.45(\mathrm{~m}, 15 \mathrm{H})$, 7.51-7.56(m, 8H), 12.64(brs, $\left.2 \mathrm{H}, \mathrm{NH}) \mathrm{ppm} ;{ }^{13} \mathrm{C} \mathrm{NMR} \mathrm{(101MHz,} \mathrm{CDCl}_{3}\right)$ : $\delta=114.39\left(\mathrm{dd}, \mathrm{J}_{\mathrm{CP}}=24 \mathrm{~Hz}, 2 \mathrm{C}\right), 123.51\left(\mathrm{dd}, \mathrm{J}_{\mathrm{CP}}=2.2 \mathrm{~Hz}, 2 \mathrm{CH}\right), 125.40(\mathrm{dd}$, $\left.\mathrm{J}_{\mathrm{CP}}=27.8 .0 \mathrm{~Hz}, 4 \mathrm{C}\right), 126.01\left(\mathrm{dd}, \mathrm{J}_{\mathrm{CP}}=3.0 \mathrm{~Hz}, 2 \mathrm{CH}\right), 128.92\left(\mathrm{dd}, \mathrm{J}_{\mathrm{CP}}=5.6 \mathrm{~Hz}\right.$, 8CH), 132.02(s, 4CH), 133.02(s, 2CH), 133.39(s, 2CH), 134.42(dd, $\left.\mathrm{J}_{\mathrm{CP}}=6.6 \mathrm{~Hz}, 8 \mathrm{CH}\right), 143.66\left(\mathrm{dd}, \mathrm{J}_{\mathrm{CP}}=7.5 \mathrm{~Hz}, 2 \mathrm{C}\right), 177.7\left(\mathrm{dd}, \mathrm{J}_{\mathrm{CP}}=7.5 \mathrm{~Hz}, \mathrm{C}\right)$ ppm. Anal. Calcd for $\mathrm{C}_{37} \mathrm{H}_{30} \mathrm{Cl}_{2} \mathrm{~N}_{2} \mathrm{P}_{2} \mathrm{Pd}$ : C, 59.90; H, 4.08; N, 3.78. found: C, 59.63; H, 4.15; N, 3.53.

2<smiles>C#[N+]c1ccccc1P</smiles>
$+\mathrm{NiCl}_{2} \underset{\mathrm{RT}, 24 \mathrm{~h}}{\stackrel{\mathrm{THF}}{\longrightarrow}}$<smiles>Cl[Ge]1(c2ccccc2)C2=C(Nc3ccccc3P1c1ccccc1)[P+](c1ccccc1)(c1ccccc1)c1ccccc1P2c1ccccc1</smiles> 
To a solution of $1 \mathbf{a}(288 \mathrm{mg}, 1 \mathrm{mmol})$ in THF $(10 \mathrm{~mL})$ was added $\mathrm{NiCl}_{2}(130 \mathrm{mg}, 1 \mathrm{mmol})$ under $\mathrm{N}_{2}$ atmosphere. The reaction mixture was stirred for $24 \mathrm{~h}$ at r.t and ${ }^{31} \mathrm{P}$ NMR signal disappeared. After filtration, residual solid was washed with THF to give a yellow solid $\mathbf{1 3}(45 \mathrm{mg}$, $14 \%)$.

13: m.p. $>240{ }^{\circ} \mathrm{C} .{ }^{31} \mathrm{P}$ NMR $(121 \mathrm{MHz}, \mathrm{MeOD}): \delta=-2.2 \mathrm{ppm} ;{ }^{1} \mathrm{H}$ NMR $(300 \mathrm{MHz}, \mathrm{MeOD}): \delta=7.35-7.39(\mathrm{t}, J=6.9 \mathrm{~Hz}, 2 \mathrm{H}), 7.52-7.61(\mathrm{~m}, 4 \mathrm{H})$, 7.74-7.83(m, 10H), 7.91-7.98(m, 12H)ppm; ${ }^{13} \mathrm{C}$ NMR (75MHz, MeOD): $\delta=95.55\left(\mathrm{AA}^{\prime} \mathrm{X}, J_{\mathrm{CP}}=6.8,100.5 \mathrm{~Hz}, 2 \mathrm{C}\right), 117.68\left(\mathrm{AA}^{\prime} \mathrm{X}, J_{\mathrm{CP}}=4.5,96.8 \mathrm{~Hz}\right.$, $2 \mathrm{C}), 118.04(\mathrm{~s}, 2 \mathrm{CH}), 124.69\left(\mathrm{dd}, J_{\mathrm{CP}}=6.8 \mathrm{~Hz}, 2 \mathrm{CH}\right), 130.54\left(\mathrm{dd}, J_{\mathrm{CP}}=6.8 \mathrm{~Hz}\right.$, $8 \mathrm{CH}), \quad 131.28\left(\mathrm{dd}, \quad J_{\mathrm{CP}}=4.5 \mathrm{~Hz}, \quad 2 \mathrm{CH}\right), \quad 134.19\left(\mathrm{dd}, J_{\mathrm{CP}}=6.0 \mathrm{~Hz}, \quad 8 \mathrm{CH}\right)$, 135.55(s, 2CH), 135.82(s, 4CH), $143.01\left(\mathrm{dd}, J_{\mathrm{CP}}=6.8 \mathrm{~Hz}, 4 \mathrm{C}\right) \mathrm{ppm}$. One

cardon is missing. HRMS calcd for $\mathrm{C}_{38} \mathrm{H}_{30} \mathrm{~N}_{2} \mathrm{P}_{2}[\mathrm{M}-2 \mathrm{Cl}]^{2+}$ : 288.0937; found : 288.0940 .

\section{References:}

[1] (a) Lygin, A. V.; de Meijere, A. Org. Lett. 2009, 11, 389-392. (b) Lygin, A. V.; de Meijere, A. J. Org. Chem. 2009, 74, 4554-4559. [2]Aluri, B. R.; Heinicke, J. Dalton Trans. 2011, 40, 211-224.

3)X-ray Crystallographic Studies of 5b; 10a; 10b; 11;12;13

X-ray Crystallographic Studies of Compound 5b:

The single crystal of $\mathbf{5 b}$ suitable for $\mathrm{X}$-ray analysis was grown in solution of dichloromethane, petroleum ether. Data collections for $\mathbf{5 b}$ 
were performed at $291.15 \mathrm{~K}$ on an oxford diffraction Gemini $\mathrm{E}$ diffractometer using graphite-monochromated $\mathrm{Mo} \mathrm{K} \alpha$ radiation $(\lambda=0.71073 \AA)$. The structure of $\mathbf{5 b}$ was solved by use of SHELXTL program. Refinement was performed on F2 anisotropically for all the non-hydrogen atoms by the full-matrix least-squares method. Crystallographic data has been deposited with the Cambridge Crystallographic Data Center. CCDC reference number: 1040476

Table 1-1 Crystal data and structure refinement for $\mathbf{5 b}$.

Empirical formula C21H18NPS

Formula weight $\quad 347.39$

Temperature/K $\quad 291.15$

Crystal system triclinic

Space group $\quad \mathrm{P}-1$

$\mathrm{a} / \AA ̊ \AA 8.3561(8)$

$\mathrm{b} / \AA 99.6321(11)$

c/A 12.1639(11)

a $/^{\circ} \quad 100.757(9)$

$\beta / /^{\circ} \quad 101.011(8)$

$\gamma /{ }^{\circ} \quad 101.963(9)$

Volume/Å3 913.67(16)

Z 2

$\rho$ calcg/cm3 1.263 
$\mu / \mathrm{mm}^{-1} \quad 2.391$

$\mathrm{F}(000) \quad 364.0$

Crystal size $/ \mathrm{mm} 3 \quad 0.2 \times 0.2 \times 0.17$

Radiation $\operatorname{CuK} \alpha(\lambda=1.54184)$

$2 \Theta$ range for data collection/ ${ }^{\circ} 7.62$ to 144.94

Index ranges $\quad-10 \leqslant \mathrm{~h} \leqslant 7,-11 \leqslant \mathrm{k} \leqslant 11,-13 \leqslant 1 \leqslant 14$

Reflections collected 6755

Independent reflections 3494 [Rint $=0.0282$, Rsigma $=0.0458]$

Data/restraints/parameters 3494/0/218

Goodness-of-fit on F2 1.029

Final $\mathrm{R}$ indexes $[\mathrm{I}>=2 \sigma(\mathrm{I})] \quad \mathrm{R} 1=0.0488, \mathrm{wR} 2=0.1216$

Final R indexes [all data] $\mathrm{R} 1=0.0669, \mathrm{wR} 2=0.1345$

Largest diff. peak/hole / e $\AA-3 \quad 0.26 /-0.28$

Table 1-2 Bond Lengths for 5b

\begin{tabular}{|c|c|c|c|c|c|}
\hline Atom & Atom & Length/Å & Atom & Atom & Length/Å \\
\hline S1 & P1 & $1.9403(9)$ & $\mathrm{C} 8$ & $\mathrm{C} 13$ & $1.389(4)$ \\
\hline P1 & $\mathrm{C} 7$ & $1.857(2)$ & C9 & $\mathrm{C} 10$ & $1.379(4)$ \\
\hline P1 & C9 & $1.792(3)$ & $\mathrm{C} 10$ & C11 & $1.391(4)$ \\
\hline P1 & $\mathrm{C} 15$ & $1.830(3)$ & C11 & $\mathrm{C} 12$ & $1.385(4)$ \\
\hline N1 & $\mathrm{C} 7$ & $1.281(3)$ & $\mathrm{C} 12$ & $\mathrm{C} 13$ & $1.392(4)$ \\
\hline N1 & $\mathrm{C} 8$ & $1.432(3)$ & $\mathrm{C} 12$ & C14 & $1.512(4)$ \\
\hline $\mathrm{C} 1$ & $\mathrm{C} 2$ & $1.384(4)$ & $\mathrm{C} 15$ & C16 & $1.497(3)$ \\
\hline
\end{tabular}




\begin{tabular}{llllll}
\hline $\mathrm{C} 1$ & $\mathrm{C} 6$ & $1.388(4)$ & $\mathrm{C} 16$ & $\mathrm{C} 17$ & $1.381(3)$ \\
$\mathrm{C} 2$ & $\mathrm{C} 3$ & $1.379(4)$ & $\mathrm{C} 16$ & $\mathrm{C} 21$ & $1.386(4)$ \\
$\mathrm{C} 3$ & $\mathrm{C} 4$ & $1.374(5)$ & $\mathrm{C} 17$ & $\mathrm{C} 18$ & $1.381(4)$ \\
$\mathrm{C} 4$ & $\mathrm{C} 5$ & $1.386(4)$ & $\mathrm{C} 18$ & $\mathrm{C} 19$ & $1.386(4)$ \\
$\mathrm{C} 5$ & $\mathrm{C} 6$ & $1.388(4)$ & $\mathrm{C} 19$ & $\mathrm{C} 20$ & $1.375(4)$ \\
$\mathrm{C} 6$ & $\mathrm{C} 7$ & $1.467(4)$ & $\mathrm{C} 20$ & $\mathrm{C} 21$ & $1.372(4)$ \\
$\mathrm{C} 8$ & $\mathrm{C} 9$ & $1.388(3)$ & & & \\
\hline
\end{tabular}

\section{Table 1-3 Bond Angles for 5b.}

\begin{tabular}{llllllll}
\hline Atom & Atom & Atom & Angle $^{\circ}$ & Atom & Atom & Atom & Angle ${ }^{\circ}$ \\
\hline C7 & P1 & S1 & $121.48(9)$ & C13 & C8 & N1 & $121.9(2)$ \\
C9 & P1 & S1 & $119.42(9)$ & C8 & C9 & P1 & $108.21(19)$ \\
C9 & P1 & C7 & $88.99(12)$ & C10 & C9 & P1 & $131.6(2)$ \\
C9 & P1 & C15 & $106.13(13)$ & C10 & C9 & C8 & $120.1(3)$ \\
C15 & P1 & S1 & $115.54(9)$ & $\mathrm{C} 9$ & $\mathrm{C} 10$ & $\mathrm{C} 11$ & $118.6(3)$ \\
C15 & P1 & C7 & $100.99(11)$ & $\mathrm{C} 12$ & $\mathrm{C} 11$ & $\mathrm{C} 10$ & $121.9(3)$ \\
$\mathrm{C} 7$ & $\mathrm{~N} 1$ & $\mathrm{C} 8$ & $112.7(2)$ & $\mathrm{C} 11$ & $\mathrm{C} 12$ & $\mathrm{C} 13$ & $119.0(3)$ \\
$\mathrm{C} 2$ & $\mathrm{C} 1$ & $\mathrm{C} 6$ & $120.7(3)$ & $\mathrm{C} 11$ & $\mathrm{C} 12$ & $\mathrm{C} 14$ & $120.6(3)$ \\
$\mathrm{C} 3$ & $\mathrm{C} 2$ & $\mathrm{C} 1$ & $120.3(3)$ & $\mathrm{C} 13$ & $\mathrm{C} 12$ & $\mathrm{C} 14$ & $120.4(3)$ \\
$\mathrm{C} 4$ & $\mathrm{C} 3$ & $\mathrm{C} 2$ & $119.6(3)$ & $\mathrm{C} 8$ & $\mathrm{C} 13$ & $\mathrm{C} 12$ & $119.3(3)$ \\
$\mathrm{C} 3$ & $\mathrm{C} 4$ & $\mathrm{C} 5$ & $120.4(3)$ & $\mathrm{C} 16$ & $\mathrm{C} 15$ & $\mathrm{P} 1$ & $114.76(17)$ \\
$\mathrm{C} 4$ & $\mathrm{C} 5$ & $\mathrm{C} 6$ & $120.6(3)$ & $\mathrm{C} 17$ & $\mathrm{C} 16$ & $\mathrm{C} 15$ & $120.8(2)$ \\
$\mathrm{C} 1$ & $\mathrm{C} 6$ & $\mathrm{C} 5$ & $118.5(3)$ & $\mathrm{C} 17$ & $\mathrm{C} 16$ & $\mathrm{C} 21$ & $118.4(3)$ \\
\hline & & & & & & & \\
\hline
\end{tabular}




\begin{tabular}{llllllll}
\hline $\mathrm{C} 1$ & $\mathrm{C} 6$ & $\mathrm{C} 7$ & $122.0(2)$ & $\mathrm{C} 21$ & $\mathrm{C} 16$ & $\mathrm{C} 15$ & $120.7(2)$ \\
$\mathrm{C} 5$ & $\mathrm{C} 6$ & $\mathrm{C} 7$ & $119.5(3)$ & $\mathrm{C} 18$ & $\mathrm{C} 17$ & $\mathrm{C} 16$ & $120.8(3)$ \\
$\mathrm{N} 1$ & $\mathrm{C} 7$ & $\mathrm{P} 1$ & $112.91(19)$ & $\mathrm{C} 17$ & $\mathrm{C} 18$ & $\mathrm{C} 19$ & $119.9(3)$ \\
$\mathrm{N} 1$ & $\mathrm{C} 7$ & $\mathrm{C} 6$ & $122.1(2)$ & $\mathrm{C} 20$ & $\mathrm{C} 19$ & $\mathrm{C} 18$ & $119.5(3)$ \\
$\mathrm{C} 6$ & $\mathrm{C} 7$ & $\mathrm{P} 1$ & $124.68(19)$ & $\mathrm{C} 21$ & $\mathrm{C} 20$ & $\mathrm{C} 19$ & $120.3(3)$ \\
$\mathrm{C} 9$ & $\mathrm{C} 8$ & $\mathrm{~N} 1$ & $117.1(2)$ & $\mathrm{C} 20$ & $\mathrm{C} 21$ & $\mathrm{C} 16$ & $121.0(3)$ \\
$\mathrm{C} 9$ & $\mathrm{C} 8$ & $\mathrm{C} 13$ & $121.0(3)$ & & & & \\
\hline
\end{tabular}

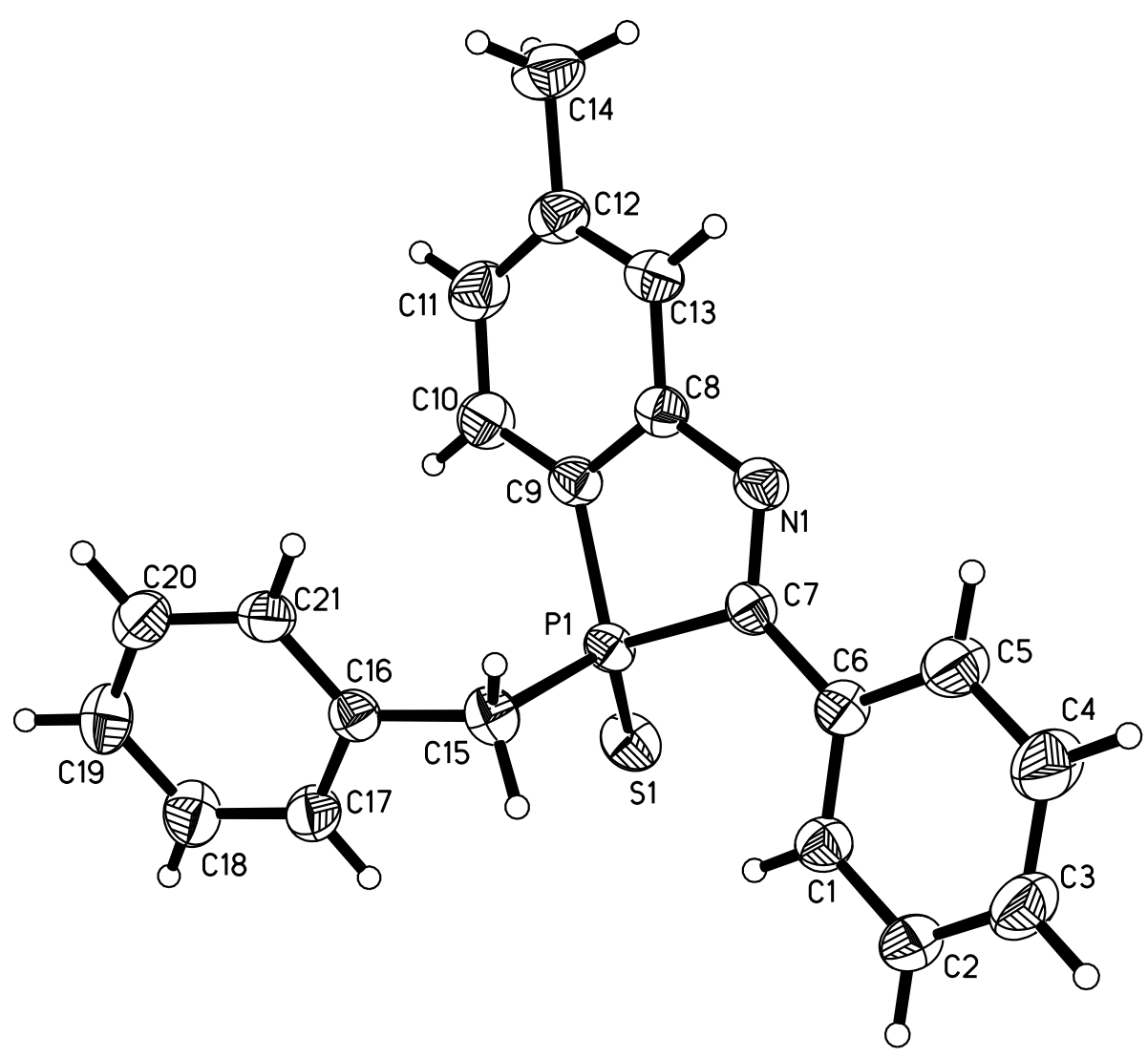


SFigure 1. ORTEP drawing of $5 \mathrm{~b}$ with $30 \%$ probability thermal ellipsoids.

X-ray Crystallographic Studies of Compound 10a:

The single crystal of 10a suitable for X-ray analysis was grown in solution of dichloromethane, hexane. Data collections for 10a were performed at 293(2) $\mathrm{K}$ on an Rigaku CrystalClear-SM Expert 2.0 diffractometer equipped with graphite monochromatic Mo $\mathrm{K} \alpha$ radiation $(\lambda=0.71073 \AA)$. The structure of $\mathbf{1 0 a}$ was solved by the direct method and refined by the full-matrix least-squares method on $\mathrm{F}$ with anisotropic thermal parameters for all non-hydrogen atoms. Crystallographic data has been deposited with the Cambridge Crystallographic Data Center. CCDC reference number: 1405477

Table 2-1 Crystal data and structure refinement for $\mathbf{1 0 a}$.

Empirical formula $\quad$ C19 H14 Au2 Cl2 N P

Formula weight $\quad 752.11$

Temperature 293(2) K

Wavelength $\quad 0.71073 \mathrm{~A}$

Crystal system, space group Triclinic, $\mathrm{P}-1$

Unit cell dimensions $\quad \mathrm{a}=9.7384(19) \mathrm{A} \quad$ alpha $=77.74(3) \mathrm{deg}$.

$\mathrm{b}=9.838(2) \mathrm{A} \quad$ beta $=78.09(3) \mathrm{deg}$.

$\mathrm{c}=10.857(2) \mathrm{A} \quad$ gamma $=80.73(3) \mathrm{deg}$.

Volume $\quad 987.0(3) \mathrm{A}^{\wedge} 3$ 
Z, Calculated density $\quad 2, \quad 2.531 \mathrm{Mg} / \mathrm{m}^{\wedge} 3$

Absorption coefficient $\quad 15.200 \mathrm{~mm}^{\wedge}-1$

$\mathrm{F}(000) \quad 684$

Crystal size $\quad 0.21 \times 0.19 \times 0.18 \mathrm{~mm}$

Theta range for data collection 1.95 to $25.50 \mathrm{deg}$.

Limiting indices $-11<=\mathrm{h}<=11,-11<=\mathrm{k}<=11,-13<=\mathrm{l}<=13$

Reflections collected / unique 10432 / 3672 [R(int) $=0.0524]$

Completeness to theta $=25.50 \quad 99.9 \%$

Absorption correction Semi-empirical from equivalents

Max. and min. transmission $\quad 0.1706$ and 0.1425

Refinement method $\quad$ Full-matrix least-squares on $\mathrm{F}^{\wedge} 2$

Data / restraints / parameters 3672 / 0 / 226

Goodness-of-fit on $\mathrm{F}^{\wedge} 2$

Final $\mathrm{R}$ indices $[\mathrm{I}>2 \operatorname{sigma}(\mathrm{I})] \quad \mathrm{R} 1=0.0450, \mathrm{wR} 2=0.1056$

$\mathrm{R}$ indices (all data) $\quad \mathrm{R} 1=0.0562, \mathrm{wR} 2=0.1124$

Largest diff. peak and hole $\quad 0.889$ and -1.525 e. $\mathrm{A}^{\wedge}-3$

Table 2-2 Bond Lengths for 10a

\begin{tabular}{llllll}
\hline Atom & Atom & Length $/ \AA$ & Atom & Atom & Length $/ \AA$ \\
\hline Au1 & P1 & $2.233(2)$ & $\mathrm{C} 8$ & H8 & 0.9300 \\
$\mathrm{Au} 1$ & $\mathrm{Cl} 1$ & $2.284(3)$ & $\mathrm{C} 9$ & $\mathrm{C} 10$ & $1.402(17)$ \\
$\mathrm{Au} 2$ & $\mathrm{C} 19$ & $1.939(13)$ & $\mathrm{C} 9$ & $\mathrm{H} 9$ & 0.9300 \\
$\mathrm{Au} 2$ & $\mathrm{Cl} 2$ & $2.243(3)$ & $\mathrm{C} 6$ & $\mathrm{C} 5$ & $1.364(14)$ \\
\hline
\end{tabular}




\begin{tabular}{llllll}
\hline P1 & C1 & $1.801(10)$ & C6 & H6 & 0.9300 \\
P1 & C7 & $1.804(9)$ & $\mathrm{C} 12$ & $\mathrm{H} 12$ & 0.9300 \\
P1 & C13 & $1.827(9)$ & $\mathrm{C} 18$ & $\mathrm{C} 17$ & $1.389(14)$ \\
$\mathrm{C} 2$ & $\mathrm{C} 1$ & $1.368(14)$ & $\mathrm{C} 5$ & $\mathrm{C} 4$ & $1.376(16)$ \\
$\mathrm{C} 2$ & $\mathrm{C} 3$ & $1.368(16)$ & $\mathrm{C} 5$ & $\mathrm{H} 5$ & 0.9300 \\
$\mathrm{C} 2$ & $\mathrm{H} 2$ & 0.9300 & $\mathrm{C} 14$ & $\mathrm{C} 15$ & $1.391(15)$ \\
$\mathrm{C} 11$ & $\mathrm{C} 10$ & $1.350(17)$ & $\mathrm{C} 9$ & $\mathrm{H} 14$ & 0.9300 \\
$\mathrm{C} 11$ & $\mathrm{C} 12$ & $1.385(15)$ & $\mathrm{C} 17$ & $\mathrm{C} 16$ & $1.349(17)$ \\
$\mathrm{C} 11$ & $\mathrm{H} 11$ & 0.9300 & $\mathrm{C} 17$ & $\mathrm{H} 17$ & 0.9300 \\
$\mathrm{C} 7$ & $\mathrm{C} 12$ & $1.376(13)$ & $\mathrm{C} 4$ & $\mathrm{C} 3$ & $1.343(15)$ \\
$\mathrm{C} 7$ & $\mathrm{C} 8$ & $1.390(13)$ & $\mathrm{C} 4$ & $\mathrm{H} 4$ & 0.9300 \\
$\mathrm{C} 19$ & $\mathrm{~N} 1$ & $1.126(14)$ & $\mathrm{C} 15$ & $\mathrm{C} 16$ & $1.380(17)$ \\
$\mathrm{C} 13$ & $\mathrm{C} 14$ & $1.367(14)$ & $\mathrm{C} 15$ & $\mathrm{H} 15$ & 0.9300 \\
$\mathrm{C} 13$ & $\mathrm{C} 18$ & $1.391(13)$ & $\mathrm{C} 3$ & $\mathrm{H} 3$ & 0.9300 \\
$\mathrm{C} 1$ & $\mathrm{C} 6$ & $1.414(13)$ & $\mathrm{C} 10$ & $\mathrm{H} 10$ & 0.9300 \\
$\mathrm{~N} 1$ & $\mathrm{C} 18$ & $1.413(14)$ & $\mathrm{C} 16$ & $\mathrm{H} 16$ & 0.9300 \\
$\mathrm{C} 8$ & $\mathrm{C} 9$ & $1.402(15)$ & $\mathrm{Au} 1$ & $\mathrm{Au} 2$ & $3.4592(10)$ \\
\hline & & & & & \\
\hline
\end{tabular}

Table 2-3 Bond Angles for 10a.

\begin{tabular}{llllllll}
\hline Atom & Atom & Atom & Angle $^{\circ}$ & Atom & Atom & Atom & Angle ${ }^{\circ}$ \\
\hline P1 & Au1 & Cl1 & $174.30(10)$ & C5 & C6 & H6 & 119.7 \\
\hline
\end{tabular}




\begin{tabular}{|c|c|c|c|c|c|c|c|}
\hline C19 & $\mathrm{Au} 2$ & $\mathrm{Cl} 2$ & $178.5(4)$ & $\mathrm{C} 1$ & C6 & H6 & 119.6 \\
\hline $\mathrm{C} 1$ & $\mathrm{P} 1$ & $\mathrm{C} 7$ & $108.5(4)$ & $\mathrm{C} 7$ & $\mathrm{C} 12$ & $\mathrm{C} 11$ & $119.9(10)$ \\
\hline $\mathrm{C} 1$ & $\mathrm{P} 1$ & $\mathrm{C} 13$ & $103.2(4)$ & $\mathrm{C} 11$ & $\mathrm{C} 12$ & $\mathrm{H} 12$ & 120.1 \\
\hline $\mathrm{C} 7$ & $\mathrm{P} 1$ & $\mathrm{C} 13$ & $106.2(4)$ & N1 & $\mathrm{C} 18$ & $\mathrm{C} 17$ & $118.0(10)$ \\
\hline $\mathrm{C} 1$ & $\mathrm{P} 1$ & $\mathrm{Au} 1$ & $114.8(3)$ & N1 & $\mathrm{C} 18$ & $\mathrm{C} 13$ & 119.2(9) \\
\hline $\mathrm{C} 7$ & $\mathrm{P} 1$ & Au1 & $108.4(3)$ & $\mathrm{C} 17$ & $\mathrm{C} 18$ & $\mathrm{C} 13$ & $122.8(10)$ \\
\hline $\mathrm{C} 13$ & $\mathrm{P} 1$ & $\mathrm{Au} 1$ & $115.2(3)$ & C6 & $\mathrm{C} 5$ & $\mathrm{C} 4$ & $118.9(10)$ \\
\hline $\mathrm{C} 1$ & $\mathrm{C} 2$ & $\mathrm{C} 3$ & $118.6(10)$ & C6 & $\mathrm{C} 5$ & $\mathrm{H} 5$ & 120.5 \\
\hline $\mathrm{C} 1$ & $\mathrm{C} 2$ & $\mathrm{H} 2$ & 120.7 & $\mathrm{C} 4$ & $\mathrm{C} 5$ & H5 & 120.5 \\
\hline $\mathrm{C} 3$ & $\mathrm{C} 2$ & $\mathrm{H} 2$ & 120.7 & $\mathrm{C} 13$ & C14 & $\mathrm{C} 15$ & $120.4(11)$ \\
\hline C10 & $\mathrm{C} 11$ & $\mathrm{C} 12$ & $120.9(12)$ & C13 & C14 & $\mathrm{H} 14$ & 119.8 \\
\hline $\mathrm{C} 10$ & $\mathrm{C} 11$ & H11 & 119.6 & $\mathrm{C} 12$ & $\mathrm{C} 7$ & $\mathrm{P} 1$ & $118.3(7)$ \\
\hline $\mathrm{C} 12$ & $\mathrm{C} 11$ & H11 & 119.6 & C16 & $\mathrm{C} 17$ & $\mathrm{C} 18$ & $118.0(11)$ \\
\hline $\mathrm{C} 12$ & $\mathrm{C} 7$ & $\mathrm{C} 8$ & $120.0(9)$ & C16 & $\mathrm{C} 17$ & $\mathrm{H} 17$ & 121.0 \\
\hline $\mathrm{C} 8$ & $\mathrm{C} 7$ & $\mathrm{P} 1$ & $121.6(8)$ & $\mathrm{C} 18$ & $\mathrm{C} 17$ & $\mathrm{H} 17$ & 121.0 \\
\hline N1 & C19 & $\mathrm{Au} 2$ & $179.6(13)$ & $\mathrm{C} 3$ & $\mathrm{C} 4$ & $\mathrm{C} 5$ & $120.6(11)$ \\
\hline C14 & $\mathrm{C} 13$ & $\mathrm{C} 18$ & $117.6(10)$ & $\mathrm{C} 3$ & $\mathrm{C} 4$ & $\mathrm{H} 4$ & 119.7 \\
\hline C14 & $\mathrm{C} 13$ & $\mathrm{P} 1$ & $123.5(8)$ & $\mathrm{C} 5$ & $\mathrm{C} 4$ & $\mathrm{H} 4$ & 119.7 \\
\hline C18 & $\mathrm{C} 13$ & $\mathrm{P} 1$ & $118.9(8)$ & C16 & C15 & $\mathrm{C} 14$ & $120.1(11)$ \\
\hline $\mathrm{C} 2$ & $\mathrm{C} 1$ & C6 & 119.2(9) & $\mathrm{C} 16$ & C15 & H15 & 120.0 \\
\hline $\mathrm{C} 2$ & $\mathrm{C} 1$ & $\mathrm{P} 1$ & $120.8(8)$ & C14 & $\mathrm{C} 15$ & H15 & 120.0 \\
\hline C6 & $\mathrm{C} 1$ & $\mathrm{P} 1$ & $119.9(7)$ & $\mathrm{C} 4$ & $\mathrm{C} 3$ & $\mathrm{C} 2$ & $121.9(11)$ \\
\hline
\end{tabular}




\begin{tabular}{llllllll}
\hline C19 & N1 & C18 & $176.6(11)$ & C4 & C3 & H3 & 119.0 \\
C7 & C8 & C9 & $120.1(10)$ & C2 & C3 & H3 & 119.0 \\
C7 & C8 & H8 & 120.0 & C11 & C10 & C9 & $120.9(11)$ \\
C9 & C8 & H8 & 120.0 & C11 & C10 & H10 & 119.6 \\
C8 & C9 & C10 & $118.2(10$ & C9 & C10 & H10 & 119.6 \\
C8 & C9 & H9 & 120.9 & C17 & C16 & C15 & $121.2(11)$ \\
C10 & C9 & H9 & 120.9 & C17 & C16 & H16 & 119.4 \\
C5 & C6 & C1 & $120.7(10)$ & C15 & C16 & H16 & 119.4 \\
\hline
\end{tabular}

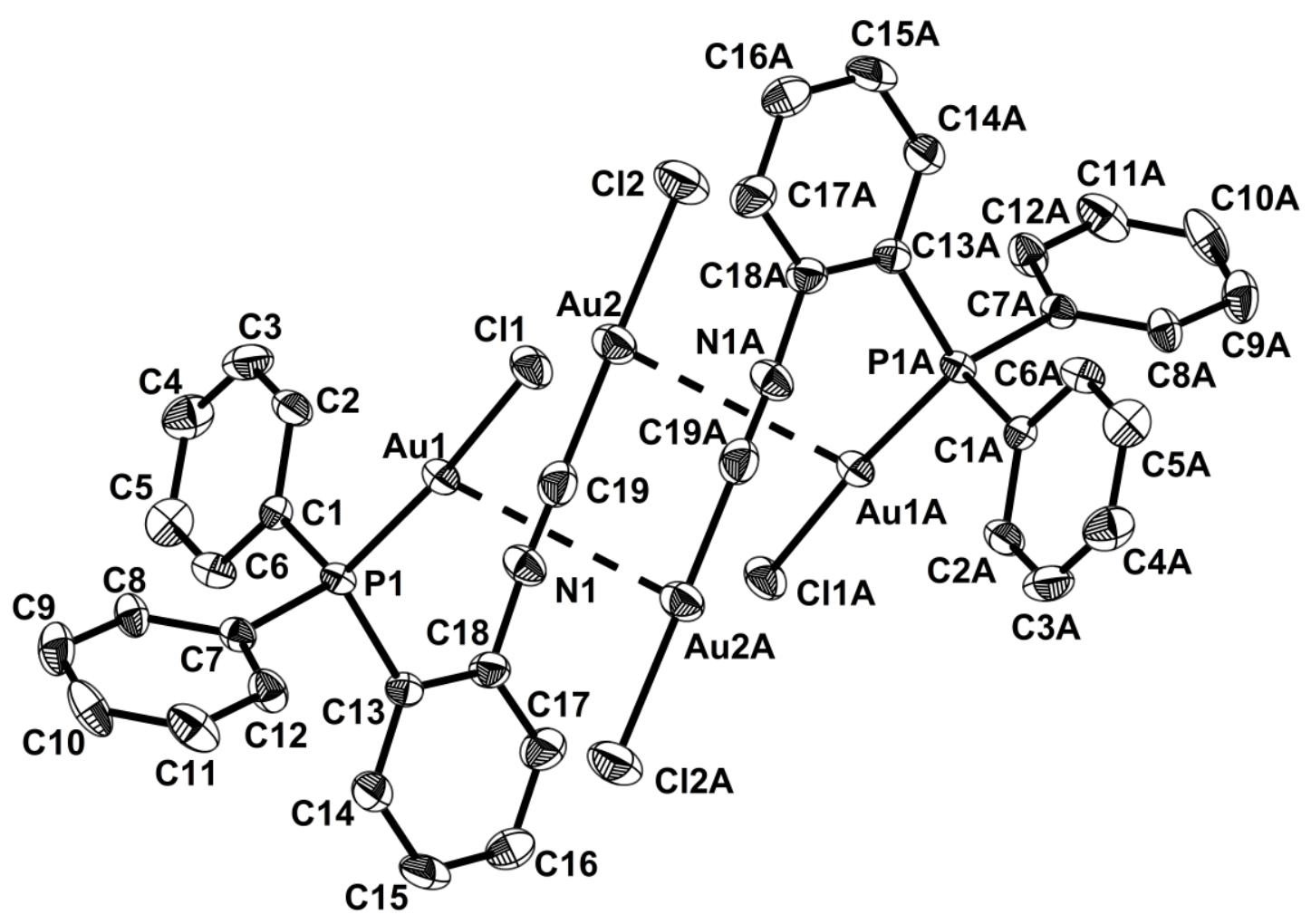

SFigure 2. ORTEP drawing of $10 a$ with $30 \%$ probability thermal ellipsoids. Hydrogen atoms have been omitted for clarity. 
X-ray Crystallographic Studies of Compound 10b:

The single crystal of $\mathbf{1 0 b}$ suitable for X-ray analysis was grown in solution of dichloromethane, hexane. Data collections for $\mathbf{1 0 b}$ were performed at $291.15 \mathrm{~K}$ on an oxford diffraction Gemini E diffractometer using graphite-monochromated Mo $\mathrm{K} \alpha$ radiation $(\lambda=0.71073 \AA)$. The structure of $\mathbf{1 0 b}$ was solved by use of SHELXTL program. Refinement was performed on F2 anisotropically for all the non-hydrogen atoms by the full-matrix least-squares method. Crystallographic data has been deposited with the Cambridge Crystallographic Data Center. CCDC reference number: 1061864

Table 3-1 Crystal data and structure refinement for $\mathbf{1 0 b}$.

Empirical formula C41H34Au4C16N2P2

Formula weight $\quad 1617.21$

Temperature/K $\quad 291.15$

Crystal system triclinic

Space group P-1
$\mathrm{a} / \AA ̊ A .5596(5)$
b/Å 10.2767(6)
c/Å 12.5902(8)
$\alpha /{ }^{\circ} \quad 100.739(5)$
$\beta /{ }^{\circ} \quad 109.865(5)$
$\gamma /{ }^{\circ} \quad 94.815(5)$ 
Volume/Å3 1128.29(12)

Z 1

$\rho$ calcg/cm3 2.380

$\mu / \mathrm{mm}^{-1} \quad 28.083$

$\mathrm{F}(000) \quad 742.0$

Crystal size $/ \mathrm{mm} 3 \quad 0.15 \times 0.14 \times 0.1$

Radiation $\operatorname{CuK} \alpha(\lambda=1.54184)$

$2 \Theta$ range for data collection/ ${ }^{\circ} 7.67$ to 134.142

Index ranges $\quad-11 \leqslant \mathrm{~h} \leqslant 10,-12 \leqslant \mathrm{k} \leqslant 10,-15 \leqslant 1 \leqslant 15$

Reflections collected 8196

Independent reflections $\quad 4028[$ Rint $=0.0537$, Rsigma $=0.0649]$

Data/restraints/parameters 4028/3/254

Goodness-of-fit on F2 1.027

Final $\mathrm{R}$ indexes $[\mathrm{I}>=2 \sigma(\mathrm{I})] \quad \mathrm{R} 1=0.0466, \mathrm{wR} 2=0.1153$

Final $\mathrm{R}$ indexes [all data] $\mathrm{R} 1=0.0534, \mathrm{wR} 2=0.1216$

Largest diff. peak/hole / e $\AA-3 \quad 2.39 /-2.45$

Table 3-2 Bond Lengths for $10 \mathrm{~b}$.

\begin{tabular}{|c|c|c|c|c|c|}
\hline Atom & Atom & Length/Å & Atom & Atom & Length/Å \\
\hline $\mathrm{Au} 1$ & $\mathrm{Au} 2$ & $3.2106(6)$ & C9 & $\mathrm{P} 1$ & $1.824(9)$ \\
\hline $\mathrm{Au} 1$ & $\mathrm{C} 1$ & $1.920(9)$ & $\mathrm{C} 10$ & $\mathrm{C} 11$ & $1.374(15)$ \\
\hline $\mathrm{Au} 1$ & $\mathrm{Cl1}$ & $2.245(2)$ & $\mathrm{C} 11$ & $\mathrm{C} 12$ & $1.352(19)$ \\
\hline $\mathrm{Au} 2$ & $\mathrm{Cl} 2$ & $2.279(3)$ & $\mathrm{C} 12$ & $\mathrm{C} 13$ & $1.389(18)$ \\
\hline
\end{tabular}




\begin{tabular}{llllll}
\hline $\mathrm{Au} 2$ & $\mathrm{P} 1$ & $2.232(2)$ & $\mathrm{C} 13$ & $\mathrm{C} 14$ & $1.387(14)$ \\
$\mathrm{C} 1$ & $\mathrm{~N} 1$ & $1.139(12)$ & $\mathrm{C} 15$ & $\mathrm{C} 16$ & $1.387(14)$ \\
$\mathrm{C} 2$ & $\mathrm{C} 3$ & $1.411(13)$ & $\mathrm{C} 15$ & $\mathrm{C} 20$ & $1.399(13)$ \\
$\mathrm{C} 2$ & $\mathrm{C} 7$ & $1.382(13)$ & $\mathrm{C} 15$ & $\mathrm{P} 1$ & $1.813(9)$ \\
$\mathrm{C} 2$ & $\mathrm{~N} 1$ & $1.401(10)$ & $\mathrm{C} 16$ & $\mathrm{C} 17$ & $1.384(14)$ \\
$\mathrm{C} 3$ & $\mathrm{C} 4$ & $1.370(13)$ & $\mathrm{C} 17$ & $\mathrm{C} 18$ & $1.364(17)$ \\
$\mathrm{C} 4$ & $\mathrm{C} 5$ & $1.382(4)$ & $\mathrm{C} 18$ & $\mathrm{C} 19$ & $1.371(18)$ \\
$\mathrm{C} 4$ & $\mathrm{C} 8$ & $1.517(13)$ & $\mathrm{C} 19$ & $\mathrm{C} 20$ & $1.394(15)$ \\
$\mathrm{C} 5$ & $\mathrm{C} 6$ & $1.387(13)$ & $\mathrm{P} 1$ & $\mathrm{C} 7^{1}$ & $1.834(9)$ \\
$\mathrm{C} 6$ & $\mathrm{C} 7$ & $1.390(12)$ & $\mathrm{C} 21$ & $\mathrm{Cl} 3^{2}$ & $1.83(2)$ \\
$\mathrm{C} 7$ & $\mathrm{P} 1{ }^{1}$ & $1.834(9)$ & $\mathrm{C} 21$ & $\mathrm{Cl} 3$ & $1.735(13)$ \\
$\mathrm{C} 9$ & $\mathrm{C} 10$ & $1.392(13)$ & $\mathrm{Cl} 3$ & $\mathrm{C} 21^{2}$ & $1.83(2)$ \\
$\mathrm{C} 9$ & $\mathrm{C} 14$ & $1.378(15)$ & & & \\
\hline $11-1-\mathrm{Y} 1$ & & & & \\
\hline
\end{tabular}

${ }^{1} 1-X, 1-Y, 1-Z ;{ }^{2}-X,-Y, 1-Z$

Table 3-3 Bond Angles for 10b

\begin{tabular}{llllllll}
\hline Atom & Atom & Atom & Angle $^{\circ}$ & Atom & Atom & Atom & Angle $^{\circ}$ \\
\hline $\mathrm{C} 1$ & $\mathrm{Au} 1$ & $\mathrm{Au} 2$ & $90.2(3)$ & $\mathrm{C} 11$ & $\mathrm{C} 10$ & $\mathrm{C} 9$ & $118.9(11)$ \\
$\mathrm{C} 1$ & $\mathrm{Au} 1$ & $\mathrm{C} 11$ & $178.7(3)$ & $\mathrm{C} 12$ & $\mathrm{C} 11$ & $\mathrm{C} 10$ & $121.5(11)$ \\
$\mathrm{C} 11$ & $\mathrm{Au} 1$ & $\mathrm{Au} 2$ & $90.22(8)$ & $\mathrm{C} 11$ & $\mathrm{C} 12$ & $\mathrm{C} 13$ & $120.6(11)$ \\
$\mathrm{C} 12$ & $\mathrm{Au} 2$ & $\mathrm{Au} 1$ & $84.27(8)$ & $\mathrm{C} 14$ & $\mathrm{C} 13$ & $\mathrm{C} 12$ & $118.4(12)$ \\
$\mathrm{P} 1$ & $\mathrm{Au} 2$ & $\mathrm{Au} 1$ & $104.17(6)$ & $\mathrm{C} 9$ & $\mathrm{C} 14$ & $\mathrm{C} 13$ & $120.8(10)$ \\
\hline
\end{tabular}




\begin{tabular}{|c|c|c|c|c|c|c|c|}
\hline $\mathrm{P} 1$ & $\mathrm{Au} 2$ & $\mathrm{Cl} 2$ & $171.51(10)$ & $\mathrm{C} 16$ & $\mathrm{C} 15$ & $\mathrm{C} 20$ & 119.6(9) \\
\hline N1 & $\mathrm{C} 1$ & Au1 & 179.1(10) & $\mathrm{C} 16$ & $\mathrm{C} 15$ & $\mathrm{P} 1$ & $122.3(7)$ \\
\hline $\mathrm{C} 7$ & $\mathrm{C} 2$ & $\mathrm{C} 3$ & $122.3(8)$ & $\mathrm{C} 20$ & $\mathrm{C} 15$ & $\mathrm{P} 1$ & $118.0(8)$ \\
\hline $\mathrm{C} 7$ & $\mathrm{C} 2$ & N1 & $120.7(8)$ & $\mathrm{C} 17$ & $\mathrm{C} 16$ & $\mathrm{C} 15$ & $119.5(10)$ \\
\hline N1 & $\mathrm{C} 2$ & $\mathrm{C} 3$ & $117.0(8)$ & $\mathrm{C} 18$ & $\mathrm{C} 17$ & $\mathrm{C} 16$ & $121.1(11)$ \\
\hline $\mathrm{C} 4$ & $\mathrm{C} 3$ & $\mathrm{C} 2$ & 118.8(9) & $\mathrm{C} 17$ & $\mathrm{C} 18$ & C19 & $119.9(11)$ \\
\hline $\mathrm{C} 3$ & $\mathrm{C} 4$ & $\mathrm{C} 5$ & $119.4(9)$ & $\mathrm{C} 18$ & C19 & $\mathrm{C} 20$ & $120.6(11)$ \\
\hline $\mathrm{C} 3$ & $\mathrm{C} 4$ & $\mathrm{C} 8$ & 119.4(9) & C19 & $\mathrm{C} 20$ & $\mathrm{C} 15$ & 119.1(11) \\
\hline C5 & $\mathrm{C} 4$ & $\mathrm{C} 8$ & 121.1(9) & $\mathrm{C} 1$ & N1 & $\mathrm{C} 2$ & $170.8(10)$ \\
\hline $\mathrm{C} 4$ & $\mathrm{C} 5$ & C6 & $121.6(8)$ & $\mathrm{C} 7^{1}$ & $\mathrm{P} 1$ & $\mathrm{Au} 2$ & $117.5(3)$ \\
\hline C5 & C6 & $\mathrm{C} 7$ & $120.1(8)$ & C9 & $\mathrm{P} 1$ & $\mathrm{Au} 2$ & $111.9(3)$ \\
\hline $\mathrm{C} 2$ & $\mathrm{C} 7$ & C6 & 117.7(8) & C9 & $\mathrm{P} 1$ & $\mathrm{C} 7^{1}$ & $104.2(4)$ \\
\hline $\mathrm{C} 2$ & $\mathrm{C} 7$ & $\mathrm{P} 1^{1}$ & $120.4(6)$ & $\mathrm{C} 15$ & $\mathrm{P} 1$ & $\mathrm{Au} 2$ & $111.0(3)$ \\
\hline C6 & $\mathrm{C} 7$ & $\mathrm{P} 1^{1}$ & $121.8(7)$ & $\mathrm{C} 15$ & $\mathrm{P} 1$ & $\mathrm{C} 7^{1}$ & $105.0(4)$ \\
\hline $\mathrm{C} 10$ & C9 & $\mathrm{P} 1$ & $119.5(8)$ & $\mathrm{C} 15$ & $\mathrm{P} 1$ & C9 & $106.3(4)$ \\
\hline C14 & C9 & $\mathrm{C} 10$ & 119.7(10) & $\mathrm{Cl} 3$ & $\mathrm{C} 21$ & $\mathrm{Cl}^{2}$ & 108.7(6) \\
\hline $\mathrm{C} 14$ & $\mathrm{C} 9$ & $\mathrm{P} 1$ & $120.8(7)$ & $\mathrm{C} 21$ & $\mathrm{Cl} 3$ & $\mathrm{C} 21^{2}$ & $71.3(6)$ \\
\hline
\end{tabular}

${ }^{1} 1-X, 1-Y, 1-Z ;{ }^{2}-X,-Y, 1-Z$ 


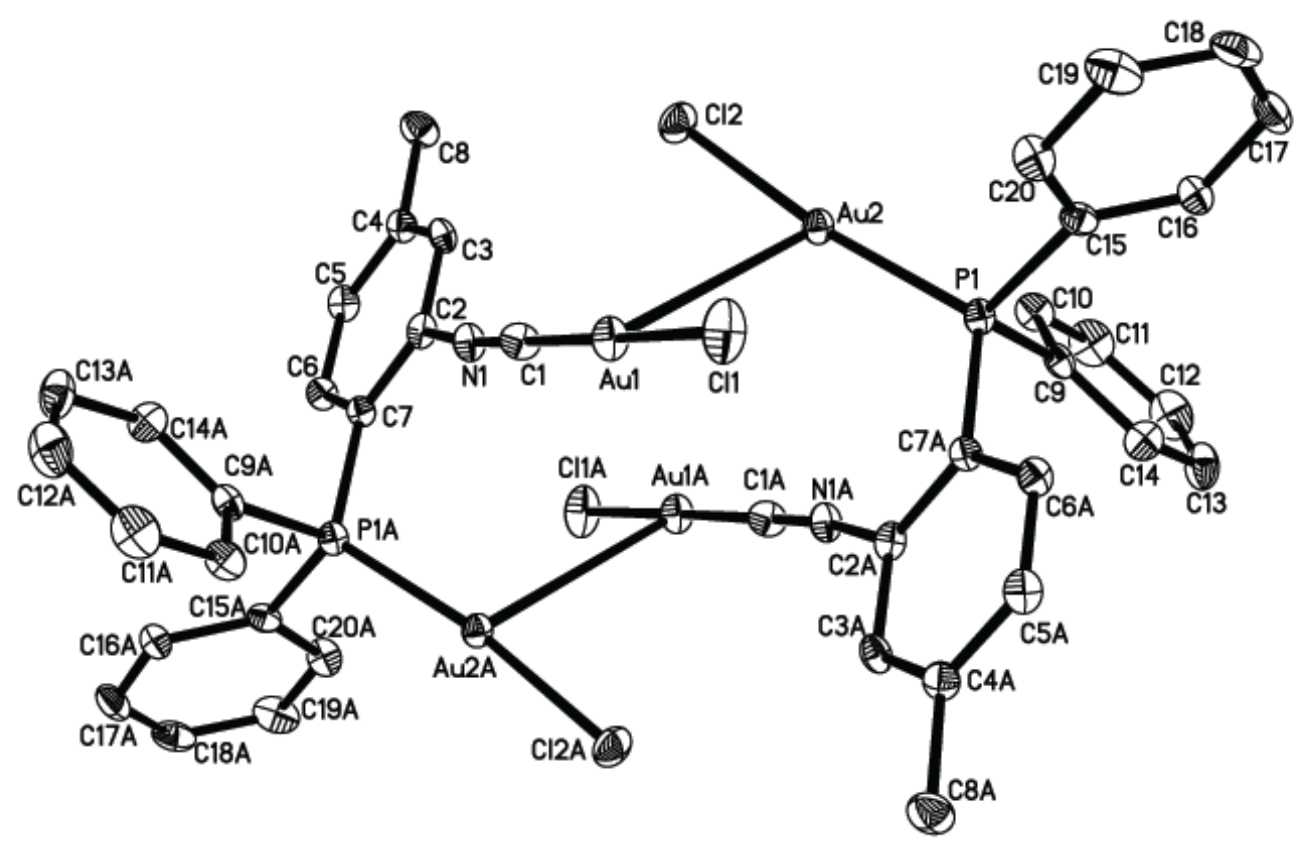

SFigure 3. ORTEP drawing of $10 \mathrm{~b}$ with $30 \%$ probability thermal ellipsoids. Hydrogen atoms have been omitted for clarity X-ray Crystallographic Studies of Compound 11:

The single crystal of $\mathbf{1 1}$ suitable for X-ray analysis was grown in solution of chloroform-d. Data collections for $\mathbf{1 1}$ were performed at $291.15 \mathrm{~K}$ on an oxford diffraction Gemini $\mathrm{E}$ diffractometer using graphite-monochromated Mo K $\alpha$ radiation $(\lambda=0.71073 \AA)$. The structure of 11 was solved by use of SHELXTL program. Refinement was performed on F2 anisotropically for all the non-hydrogen atoms by the full-matrix least-squares method. Crystallographic data has been deposited with the Cambridge Crystallographic Data Center. CCDC reference number: $\mathbf{1 4 0 1 5 3 8}$ 
Table 4-1 Crystal data and structure refinement for 11 .

Empirical formula $\mathrm{C} 40 \mathrm{H} 34 \mathrm{Au} 2 \mathrm{Cl6F} 6 \mathrm{~N} 2 \mathrm{O} 6 \mathrm{P} 2 \mathrm{~S} 2$

Formula weight $\quad 1485.38$

Temperature/K $\quad 291.15$

Crystal system monoclinic

Space group $\quad \mathrm{P} 21 / \mathrm{c}$

a/Å 9.9220(3)

$\mathrm{b} / \AA \quad 13.4139(3)$

c/Å 18.8864(4)

a ${ }^{\circ} \quad 90$

$\beta /{ }^{\circ} \quad 94.357(2)$

$\gamma /{ }^{\circ} \quad 90$

Volume/Å3 2506.38(10)

Z 2

$\rho$ calcg/cm3 1.968

$\mu / \mathrm{mm}^{-1} \quad 15.777$

$\mathrm{F}(000) \quad 1424.0$

Crystal size $/ \mathrm{mm} 3 \quad 0.14 \times 0.1 \times 0.05$

Radiation $\operatorname{CuK} \alpha(\lambda=1.54184)$

$2 \Theta$ range for data collection $/{ }^{\circ} 8.092$ to 144.796

Index ranges $\quad-11 \leqslant \mathrm{~h} \leqslant 12,-16 \leqslant \mathrm{k} \leqslant 15,-23 \leqslant 1 \leqslant 15$

Reflections collected 9626 
Independent reflections $\quad 4848[$ Rint $=0.0508$, Rsigma $=0.0691]$

Data/restraints/parameters 4848/0/301

Goodness-of-fit on F2 1.035

Final $R$ indexes $[\mathrm{I}>=2 \sigma(\mathrm{I})] \quad \mathrm{R} 1=0.0496, \mathrm{wR} 2=0.1284$

Final $\mathrm{R}$ indexes [all data] $\mathrm{R} 1=0.0594, \mathrm{wR} 2=0.1405$

Largest diff. peak/hole / e $\AA-3 \quad$ 2.05/-1.04

Table 4-2 Bond Lengths for 11

\begin{tabular}{|c|c|c|c|c|c|}
\hline Atom & Atom & Length/Å & Atom & Atom & Length/Å \\
\hline Au 1 & Au11 & $3.0007(6)$ & $\mathrm{C} 13$ & $\mathrm{C} 18$ & $1.387(11)$ \\
\hline $\mathrm{Au} 1$ & N11 & $2.130(6)$ & $\mathrm{C} 13$ & $\mathrm{P} 1$ & $1.833(7)$ \\
\hline $\mathrm{Au} 1$ & $\mathrm{P} 1$ & $2.2427(17)$ & C14 & $\mathrm{C} 15$ & $1.387(11)$ \\
\hline $\mathrm{C} 1$ & $\mathrm{C} 2$ & $1.377(12)$ & $\mathrm{C} 15$ & $\mathrm{C} 16$ & $1.357(13)$ \\
\hline $\mathrm{C} 1$ & C6 & $1.390(11)$ & C16 & $\mathrm{C} 17$ & $1.383(13)$ \\
\hline $\mathrm{C} 1$ & $\mathrm{P} 1$ & $1.817(8)$ & $\mathrm{C} 17$ & $\mathrm{C} 18$ & $1.407(11)$ \\
\hline $\mathrm{C} 2$ & $\mathrm{C} 3$ & $1.366(14)$ & $\mathrm{C} 18$ & N1 & $1.433(9)$ \\
\hline $\mathrm{C} 3$ & $\mathrm{C} 4$ & $1.389(16)$ & N1 & $\mathrm{Au} 1^{1}$ & $2.130(6)$ \\
\hline $\mathrm{C} 4$ & $\mathrm{C} 5$ & $1.365(16)$ & C19 & $\mathrm{F} 1$ & $1.320(19)$ \\
\hline $\mathrm{C} 5$ & C6 & $1.392(14)$ & C19 & $\mathrm{F} 2$ & $1.312(14)$ \\
\hline $\mathrm{C} 7$ & $\mathrm{C} 8$ & $1.394(11)$ & C19 & $\mathrm{F} 3$ & $1.284(19)$ \\
\hline $\mathrm{C} 7$ & $\mathrm{C} 12$ & $1.374(12)$ & C19 & $\mathrm{S} 1$ & $1.806(11)$ \\
\hline $\mathrm{C} 7$ & P1 & $1.808(7)$ & O1 & $\mathrm{S} 1$ & $1.406(9)$ \\
\hline C8 & C9 & $1.382(12)$ & $\mathrm{O} 2$ & $\mathrm{~S} 1$ & $1.417(10)$ \\
\hline
\end{tabular}




\begin{tabular}{llllll}
\hline $\mathrm{C} 9$ & $\mathrm{C} 10$ & $1.377(15)$ & $\mathrm{O} 3$ & $\mathrm{~S} 1$ & $1.410(10)$ \\
$\mathrm{C} 10$ & $\mathrm{C} 11$ & $1.367(17)$ & $\mathrm{C} 20$ & $\mathrm{C} 11$ & $1.740(13)$ \\
$\mathrm{C} 11$ & $\mathrm{C} 12$ & $1.398(14)$ & $\mathrm{C} 20$ & $\mathrm{C} 2$ & $1.729(14)$ \\
$\mathrm{C} 13$ & $\mathrm{C} 14$ & $1.387(11)$ & $\mathrm{C} 20$ & $\mathrm{Cl} 3$ & $1.710(13)$ \\
\hline${ }^{1}-\mathrm{X}, 1-\mathrm{Y},-\mathrm{Z}$ & & & &
\end{tabular}

Table 4-3 Bond Angles for 11.

\begin{tabular}{|c|c|c|c|c|c|c|c|}
\hline Atom & Atom & Atom & Angle ${ }^{\circ}$ & Atom & Atom & Atom & Angle ${ }^{\circ}$ \\
\hline $\mathrm{N} 1^{1}$ & $\mathrm{Au} 1$ & $\mathrm{Au} 1^{1}$ & $95.14(18)$ & $\mathrm{C} 13$ & $\mathrm{C} 18$ & $\mathrm{C} 17$ & $120.0(7)$ \\
\hline $\mathrm{C} 5$ & $\mathrm{C} 4$ & $\mathrm{C} 3$ & 119.7(9) & $\mathrm{C} 7$ & $\mathrm{P} 1$ & $\mathrm{C} 13$ & $105.9(3)$ \\
\hline $\mathrm{C} 4$ & $\mathrm{C} 5$ & C6 & $119.7(10)$ & $\mathrm{C} 13$ & $\mathrm{P} 1$ & $\mathrm{Au} 1$ & $114.0(2)$ \\
\hline $\mathrm{C} 1$ & C6 & $\mathrm{C} 5$ & $120.2(9)$ & $\mathrm{F} 1$ & C19 & $\mathrm{S} 1$ & $110.3(10)$ \\
\hline $\mathrm{C} 8$ & $\mathrm{C} 7$ & $\mathrm{P} 1$ & $120.7(6)$ & $\mathrm{F} 2$ & C19 & $\mathrm{F} 1$ & $107.0(14)$ \\
\hline $\mathrm{C} 12$ & $\mathrm{C} 7$ & $\mathrm{C} 8$ & $118.5(8)$ & $\mathrm{F} 2$ & C19 & $\mathrm{S} 1$ & $110.9(9)$ \\
\hline $\mathrm{C} 12$ & $\mathrm{C} 7$ & $\mathrm{P} 1$ & $120.8(6)$ & F3 & C19 & $\mathrm{F} 1$ & $108.0(13)$ \\
\hline C9 & $\mathrm{C} 8$ & $\mathrm{C} 7$ & 120.2(9) & $\mathrm{F} 3$ & C19 & $\mathrm{F} 2$ & $109.4(14)$ \\
\hline $\mathrm{C} 10$ & C9 & $\mathrm{C} 8$ & $120.5(9)$ & $\mathrm{F} 3$ & C19 & $\mathrm{S} 1$ & 111.1(12) \\
\hline C11 & $\mathrm{C} 10$ & C9 & 120.2(9) & $\mathrm{O} 1$ & S1 & C19 & $103.1(7)$ \\
\hline $\mathrm{C} 10$ & $\mathrm{C} 11$ & $\mathrm{C} 12$ & $119.3(10)$ & $\mathrm{O} 1$ & S1 & $\mathrm{O} 2$ & $111.7(7)$ \\
\hline C7 & $\mathrm{C} 12$ & $\mathrm{C} 11$ & 121.3(9) & $\mathrm{O} 1$ & S1 & $\mathrm{O} 3$ & $114.3(8)$ \\
\hline C14 & $\mathrm{C} 13$ & $\mathrm{P} 1$ & $118.5(6)$ & $\mathrm{O} 2$ & S1 & C19 & $101.9(7)$ \\
\hline $\mathrm{C} 1$ & $\mathrm{C} 13$ & C14 & $119.0(7)$ & $\mathrm{O} 3$ & S1 & C19 & $104.6(7)$ \\
\hline C18 & $\mathrm{C} 13$ & $\mathrm{P} 1$ & $122.4(6)$ & $\mathrm{O} 3$ & S1 & $\mathrm{O} 2$ & $118.7(8)$ \\
\hline
\end{tabular}




\begin{tabular}{llllllll}
\hline $\mathrm{C} 15$ & $\mathrm{C} 14$ & $\mathrm{C} 13$ & $120.9(8)$ & $\mathrm{Cl} 2$ & $\mathrm{C} 20$ & $\mathrm{C} 11$ & $109.0(7)$ \\
$\mathrm{C} 16$ & $\mathrm{C} 15$ & $\mathrm{C} 14$ & $119.7(8)$ & $\mathrm{C} 13$ & $\mathrm{C} 20$ & $\mathrm{C} 11$ & $110.3(7)$ \\
$\mathrm{C} 15$ & $\mathrm{C} 16$ & $\mathrm{C} 17$ & $121.3(8)$ & $\mathrm{Cl} 3$ & $\mathrm{C} 20$ & $\mathrm{C} 22$ & $108.0(8)$ \\
$\mathrm{C} 16$ & $\mathrm{C} 17$ & $\mathrm{C} 18$ & $119.0(8)$ & & & & \\
\hline
\end{tabular}

${ }^{1}-\mathrm{X}, 1-\mathrm{Y},-\mathrm{Z}$

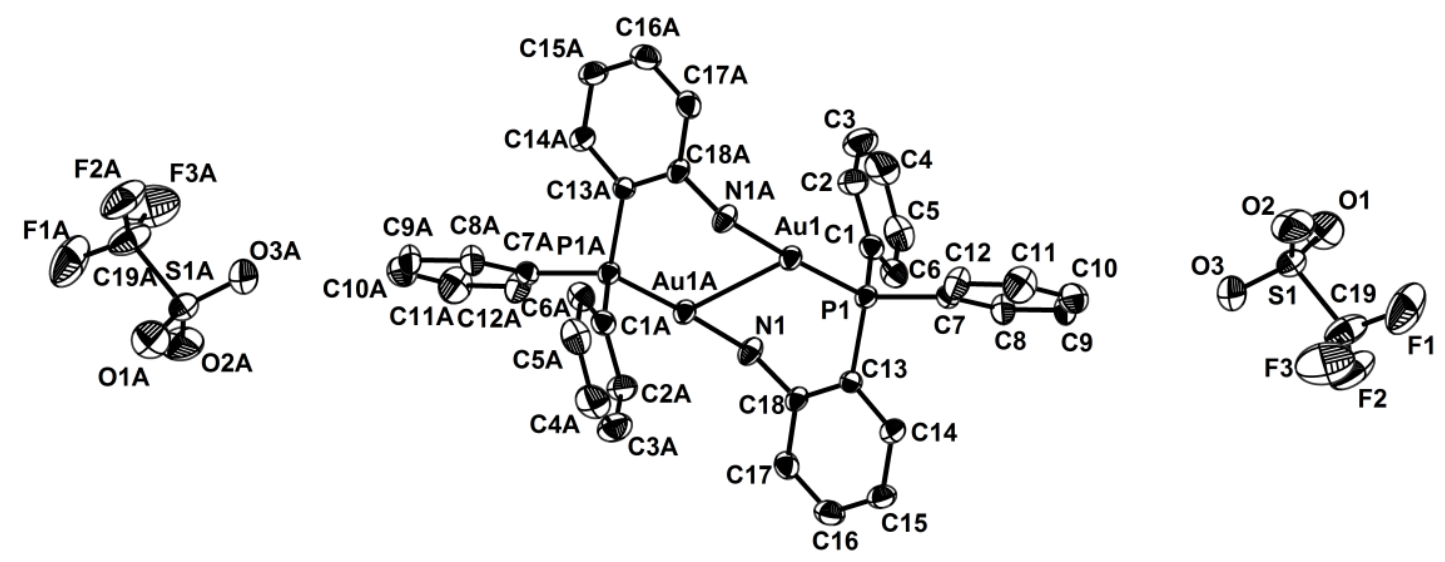

SFigure 4. ORTEP drawing of 11 with $30 \%$ probability thermal ellipsoids. Hydrogen atoms have been omitted for clarity.

X-ray Crystallographic Studies of Compound 12:

The single crystal of $\mathbf{1 2}$ suitable for X-ray analysis was grown in solution of chloroform and hexane. Data collections for 12 were performed at 291.15 K on an oxford diffraction Gemini E diffractometer using graphite-monochromated Mo $\mathrm{K} \alpha$ radiation $(\lambda=0.71073 \AA)$. The 
structure of 12 was solved by use of SHELXTL program. Refinement was performed on F2 anisotropically for all the non-hydrogen atoms by the full-matrix least-squares method. Crystallographic data has been deposited with the Cambridge Crystallographic Data Center. CCDC reference number: $\mathbf{1 0 6 3 7 0 8}$

Table 5-1 Crystal data and structure refinement for 12.

Identification code 20150598

Empirical formula $\quad \mathrm{C} 38 \mathrm{H} 31 \mathrm{Cl} 5 \mathrm{~N} 2 \mathrm{P} 2 \mathrm{Pd}$

Formula weight 861.24

Temperature/K 291.15

Crystal system monoclinic

Space group P21/n

$\mathrm{a} / \AA ̊ 10.7584(2)$

b/Å 16.6336(3)

c/A 21.6767(3)

a $/{ }^{\circ} 90$

$\beta /{ }^{\circ} \quad 103.1612(18)$

$\gamma /{ }^{\circ} 90$

Volume/Å3 3777.18(12)

Z 4

$\rho$ calcg/cm3 $\quad 1.514$

$\mu / \mathrm{mm}^{-1} \quad 8.254$ 
$\mathrm{F}(000) \quad 1736.0$

Crystal size $/ \mathrm{mm} 3 \quad 0.17 \times 0.17 \times 0.1$

Radiation $\quad \mathrm{CuK} \alpha(\lambda=1.54184)$

$2 \Theta$ range for data collection/ ${ }^{\circ} \quad 6.766$ to 134.172

Index ranges $\quad-12 \leqslant \mathrm{~h} \leqslant 10,-17 \leqslant \mathrm{k} \leqslant 19,-20 \leqslant 1 \leqslant 25$

Reflections collected 14061

Independent reflections $6719[$ Rint $=0.0270$, Rsigma $=0.0404]$

Data/restraints/parameters $\quad 6719 / 1 / 441$

Goodness-of-fit on F2 1.016

Final R indexes $[\mathrm{I}>=2 \sigma(\mathrm{I})] \mathrm{R} 1=0.0472, \mathrm{wR} 2=0.1333$

Final $\mathrm{R}$ indexes [all data] $\mathrm{R} 1=0.0622, \mathrm{wR} 2=0.1467$

Largest diff. peak/hole / e $\AA-3 \quad 1.41 /-0.87$

Table 5-2 Bond Lengths for 12.

\begin{tabular}{cccccc}
\hline Atom & Atom & Length/A & Atom & Atom & Length/A \\
\hline $\mathrm{C} 1$ & $\mathrm{C} 2$ & $1.383(7)$ & $\mathrm{C} 20$ & $\mathrm{~N} 2$ & $1.422(7)$ \\
$\mathrm{C} 1$ & $\mathrm{C} 6$ & $1.368(6)$ & $\mathrm{C} 21$ & $\mathrm{C} 22$ & $1.368(10)$ \\
$\mathrm{C} 1$ & $\mathrm{P} 1$ & $1.824(4)$ & $\mathrm{C} 22$ & $\mathrm{C} 23$ & $1.373(10)$ \\
$\mathrm{C} 2$ & $\mathrm{C} 3$ & $1.362(7)$ & $\mathrm{C} 23$ & $\mathrm{C} 24$ & $1.390(9)$ \\
$\mathrm{C} 3$ & $\mathrm{C} 4$ & $1.378(8)$ & $\mathrm{C} 24$ & $\mathrm{C} 25$ & $1.397(7)$ \\
$\mathrm{C} 4$ & $\mathrm{C} 5$ & $1.360(9)$ & $\mathrm{C} 25$ & $\mathrm{P} 2$ & $1.807(5)$ \\
$\mathrm{C} 5$ & $\mathrm{C} 6$ & $1.387(7)$ & $\mathrm{C} 26$ & $\mathrm{C} 27$ & $1.378(9)$
\end{tabular}




\begin{tabular}{|c|c|c|c|c|c|}
\hline $\mathrm{C} 7$ & $\mathrm{C} 8$ & $1.389(7)$ & $\mathrm{C} 26$ & C31 & $1.377(7)$ \\
\hline $\mathrm{C} 7$ & C12 & $1.372(7)$ & $\mathrm{C} 26$ & $\mathrm{P} 2$ & $1.815(5)$ \\
\hline $\mathrm{C} 7$ & $\mathrm{P} 1$ & $1.820(5)$ & $\mathrm{C} 27$ & C28 & $1.394(10)$ \\
\hline $\mathrm{C} 8$ & C9 & $1.387(8)$ & $\mathrm{C} 28$ & C29 & $1.407(12)$ \\
\hline C9 & $\mathrm{C} 10$ & $1.361(8)$ & $\mathrm{C} 29$ & C30 & $1.332(11)$ \\
\hline C10 & C11 & $1.363(8)$ & C30 & C31 & $1.376(9)$ \\
\hline C11 & C12 & $1.384(7)$ & C32 & C33 & $1.389(7)$ \\
\hline C13 & C14 & $1.393(7)$ & C32 & C37 & $1.397(7)$ \\
\hline C13 & C18 & $1.403(7)$ & C32 & $\mathrm{P} 2$ & $1.817(5)$ \\
\hline C13 & $\mathrm{P} 1$ & $1.813(5)$ & C33 & C34 & $1.373(9)$ \\
\hline C14 & $\mathrm{C} 15$ & $1.369(8)$ & C34 & C35 & $1.375(10)$ \\
\hline C15 & C16 & $1.367(9)$ & C35 & C36 & $1.355(9)$ \\
\hline C16 & C17 & $1.387(9)$ & C36 & C37 & $1.376(8)$ \\
\hline C17 & C18 & $1.386(7)$ & $\mathrm{Cl1}$ & Pd1 & $2.3187(13)$ \\
\hline C18 & N1 & $1.415(6)$ & $\mathrm{P} 1$ & Pd1 & $2.2821(11)$ \\
\hline C19 & N1 & $1.330(6)$ & $\mathrm{P} 2$ & Pd1 & $2.3188(11)$ \\
\hline C19 & N2 & $1.329(6)$ & C38 & $\mathrm{Cl3}$ & $1.751(6)$ \\
\hline C19 & Pd1 & $2.003(5)$ & C38 & $\mathrm{Cl} 4$ & $1.730(7)$ \\
\hline $\mathrm{C} 20$ & C21 & $1.390(7)$ & C38 & $\mathrm{Cl} 5$ & $1.730(6)$ \\
\hline $\mathrm{C} 20$ & $\mathrm{C} 25$ & $1.388(7)$ & & & \\
\hline
\end{tabular}

Table 5-3 Bond Angles for 12. 


\begin{tabular}{|c|c|c|c|c|c|c|c|}
\hline Atom & Atom & Atom & Angle $/^{\circ}$ & Atom & Atom & Atom & Angle ${ }^{\circ}$ \\
\hline $\mathrm{C} 2$ & $\mathrm{C} 1$ & P1 & $119.5(3)$ & $\mathrm{C} 27$ & $\mathrm{C} 26$ & $\mathrm{P} 2$ & $119.5(4)$ \\
\hline C6 & $\mathrm{C} 1$ & $\mathrm{C} 2$ & $119.8(4)$ & C31 & $\mathrm{C} 26$ & $\mathrm{C} 27$ & $119.2(6)$ \\
\hline C6 & $\mathrm{C} 1$ & $\mathrm{P} 1$ & $120.6(4)$ & C31 & $\mathrm{C} 26$ & $\mathrm{P} 2$ & $121.2(5)$ \\
\hline $\mathrm{C} 3$ & $\mathrm{C} 2$ & $\mathrm{C} 1$ & $120.2(5)$ & $\mathrm{C} 26$ & $\mathrm{C} 27$ & $\mathrm{C} 28$ & $120.6(7)$ \\
\hline $\mathrm{C} 2$ & $\mathrm{C} 3$ & $\mathrm{C} 4$ & $120.5(5)$ & $\mathrm{C} 27$ & $\mathrm{C} 28$ & C29 & $117.9(8)$ \\
\hline $\mathrm{C} 5$ & $\mathrm{C} 4$ & $\mathrm{C} 3$ & $119.2(5)$ & $\mathrm{C} 30$ & $\mathrm{C} 29$ & $\mathrm{C} 28$ & 121.1(7) \\
\hline $\mathrm{C} 4$ & $\mathrm{C} 5$ & C6 & $121.1(5)$ & $\mathrm{C} 29$ & $\mathrm{C} 30$ & C31 & $120.7(7)$ \\
\hline $\mathrm{C} 1$ & C6 & $\mathrm{C} 5$ & $119.2(5)$ & $\mathrm{C} 30$ & $\mathrm{C} 31$ & $\mathrm{C} 26$ & $120.5(7)$ \\
\hline $\mathrm{C} 8$ & $\mathrm{C} 7$ & P1 & $119.0(4)$ & C33 & $\mathrm{C} 32$ & C37 & $118.3(5)$ \\
\hline $\mathrm{C} 12$ & $\mathrm{C} 7$ & $\mathrm{C} 8$ & $119.2(5)$ & C33 & $\mathrm{C} 32$ & $\mathrm{P} 2$ & $117.3(4)$ \\
\hline $\mathrm{C} 12$ & $\mathrm{C} 7$ & P1 & $121.8(4)$ & C37 & $\mathrm{C} 32$ & $\mathrm{P} 2$ & $124.4(4)$ \\
\hline C9 & $\mathrm{C} 8$ & $\mathrm{C} 7$ & $119.6(5)$ & C34 & $\mathrm{C} 33$ & C 32 & $120.6(6)$ \\
\hline $\mathrm{C} 10$ & C9 & $\mathrm{C} 8$ & $120.5(5)$ & C33 & $\mathrm{C} 34$ & $\mathrm{C} 35$ & $120.1(6)$ \\
\hline C9 & $\mathrm{C} 10$ & C11 & $120.1(5)$ & C36 & C35 & C34 & $120.2(6)$ \\
\hline $\mathrm{C} 10$ & C11 & $\mathrm{C} 12$ & $120.3(5)$ & C35 & $\mathrm{C} 36$ & $\mathrm{C} 37$ & $120.8(6)$ \\
\hline $\mathrm{C} 7$ & $\mathrm{C} 12$ & C11 & $120.3(5)$ & $\mathrm{C} 36$ & C37 & $\mathrm{C} 32$ & $120.1(5)$ \\
\hline $\mathrm{C} 14$ & $\mathrm{C} 13$ & $\mathrm{C} 18$ & $118.0(5)$ & C19 & N1 & $\mathrm{C} 18$ & $129.2(4)$ \\
\hline $\mathrm{C} 14$ & $\mathrm{C} 13$ & $\mathrm{P} 1$ & $122.6(4)$ & C19 & $\mathrm{N} 2$ & $\mathrm{C} 20$ & $127.5(5)$ \\
\hline C18 & $\mathrm{C} 13$ & P1 & $119.3(4)$ & $\mathrm{C} 1$ & $\mathrm{P} 1$ & Pd1 & $120.59(15)$ \\
\hline $\mathrm{C} 15$ & C14 & $\mathrm{C} 13$ & $121.3(5)$ & $\mathrm{C} 7$ & $\mathrm{P} 1$ & $\mathrm{C} 1$ & $106.7(2)$ \\
\hline
\end{tabular}




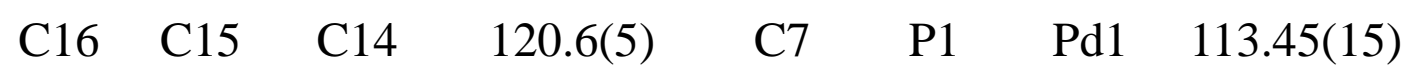

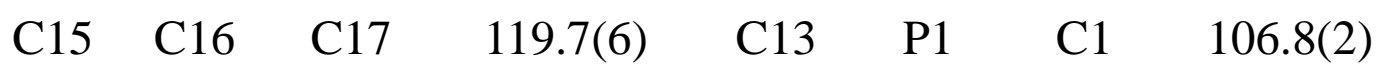

$\begin{array}{llllllll}\mathrm{C} 18 & \mathrm{C} 17 & \mathrm{C} 16 & 120.4(5) & \mathrm{C} 13 & \mathrm{P} 1 & \mathrm{C} 7 & 104.6(2)\end{array}$

$\begin{array}{llllllll}\mathrm{C} 13 & \mathrm{C} 18 & \mathrm{~N} 1 & 122.7(4) & \mathrm{C} 13 & \mathrm{P} 1 & \mathrm{Pd} 1 & 103.30(14)\end{array}$

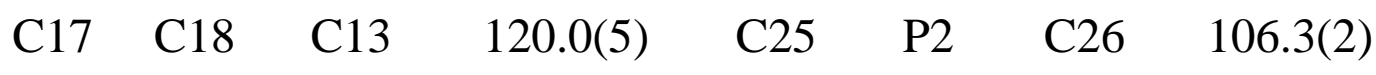

\begin{tabular}{llllllll}
\hline $\mathrm{C} 17$ & $\mathrm{C} 18$ & $\mathrm{~N} 1$ & $117.1(4)$ & $\mathrm{C} 25$ & $\mathrm{P} 2$ & $\mathrm{C} 32$ & $106.6(2)$
\end{tabular}

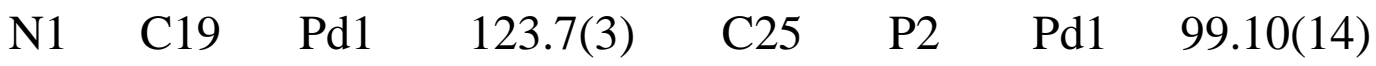

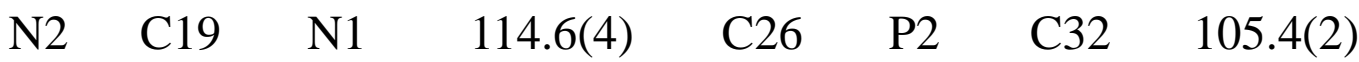

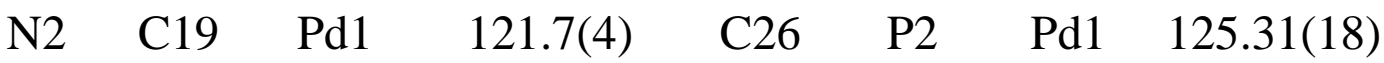

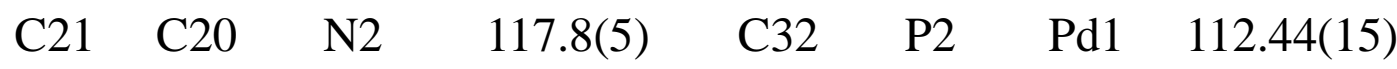

$\begin{array}{llllllll}\mathrm{C} 25 & \mathrm{C} 20 & \mathrm{C} 21 & 120.3(5) & \mathrm{C} 19 & \mathrm{Pd} 1 & \mathrm{C} 11 & 176.80(14)\end{array}$

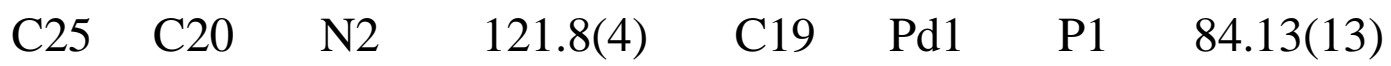

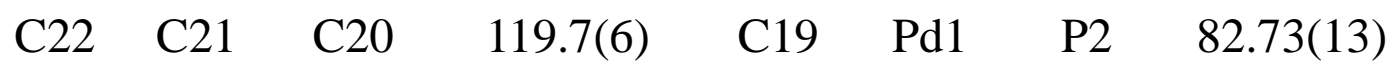

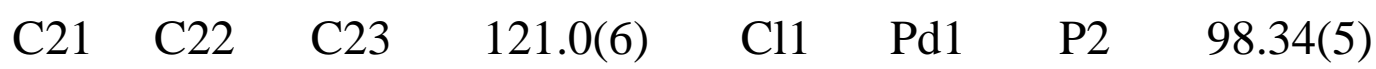

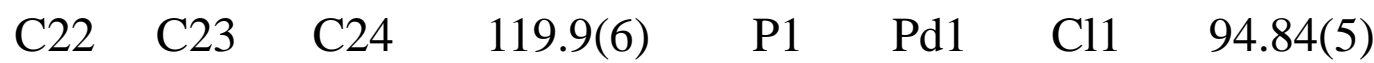

$\begin{array}{llllllll}\mathrm{C} 23 & \mathrm{C} 24 & \mathrm{C} 25 & 119.7(6) & \mathrm{P} 1 & \mathrm{Pd} 1 & \mathrm{P} 2 & 166.81(5)\end{array}$

$\begin{array}{llllllll}\mathrm{C} 20 & \mathrm{C} 25 & \mathrm{C} 24 & 119.3(5) & \mathrm{Cl} 4 & \mathrm{C} 38 & \mathrm{Cl} 3 & 110.6(4)\end{array}$

$\begin{array}{llllllll}\mathrm{C} 20 & \mathrm{C} 25 & \mathrm{P} 2 & 117.9(4) & \mathrm{Cl} 4 & \mathrm{C} 38 & \mathrm{Cl} 5 & 112.0(4)\end{array}$

$\begin{array}{llllllll}\mathrm{C} 24 & \mathrm{C} 25 & \mathrm{P} 2 & 122.7(4) & \mathrm{Cl} 5 & \mathrm{C} 38 & \mathrm{C} 13 & 109.0(4)\end{array}$ 
(1) $\mathrm{Cl} 2$

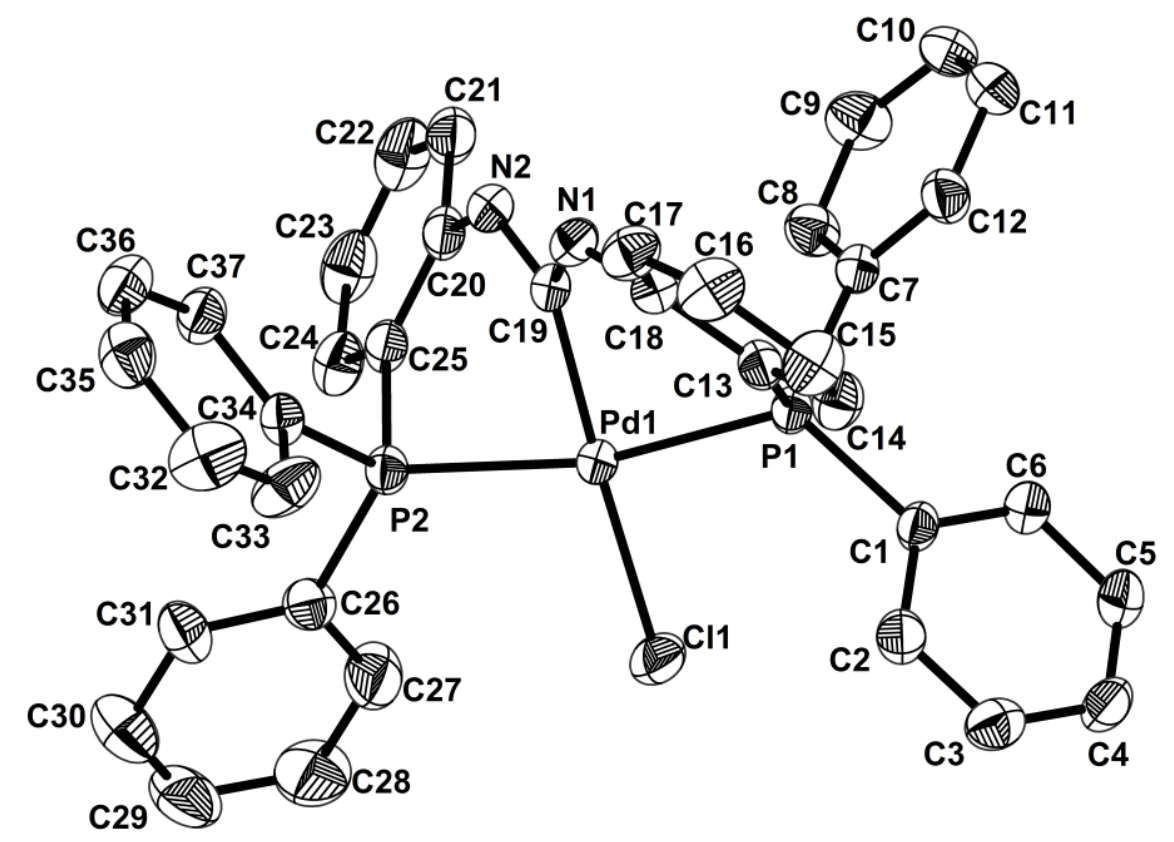

SFigure 5. ORTEP drawing of 12 with $30 \%$ probability thermal ellipsoids. Hydrogen atoms have been omitted for clarity.

X-ray Crystallographic Studies of Compound 13:

The single crystal of $\mathbf{1 3}$ suitable for X-ray analysis was grown in solution of ethanol and dichloromethane and hexane. Data collections for 13 were performed at $291.15 \mathrm{~K}$ on an oxford diffraction Gemini $\mathrm{E}$ diffractometer using graphite-monochromated $\mathrm{Mo} \mathrm{K \alpha}$ radiation $(\lambda=0.71073 \AA)$. The structure of 13 was solved by use of SHELXTL program. Refinement was performed on F2 anisotropically for all the non-hydrogen atoms by the full-matrix least-squares method. Crystallographic data has been deposited with the Cambridge Crystallographic Data Center. CCDC reference number: 1405478 
Table 6-1 Crystal data and structure refinement for 13.

Empirical formula $\mathrm{C} 38 \mathrm{H} 30 \mathrm{Cl} 2 \mathrm{~N} 2 \mathrm{P} 2$

Formula weight $\quad 647.48$

Temperature/K $\quad 291.15$

Crystal system monoclinic

Space group $\mathrm{P} 21 / \mathrm{c}$

$\mathrm{a} / \mathrm{A}$ 14.5972(3)

$\mathrm{b} / \AA \quad 14.7429(3)$

c/Å 18.6732(4)

a ${ }^{\circ} \quad 90$

$\beta /{ }^{\circ} \quad 106.922(3)$

$\gamma /{ }^{\circ} \quad 90$

Volume/Å3 3844.57(15)

Z 4

$\rho$ calcg/cm3 1.119

$\mu / \mathrm{mm}-1 \quad 2.499$

$\mathrm{F}(000) \quad 1344.0$

Crystal size $/ \mathrm{mm} 3 \quad 0.18 \times 0.15 \times 0.1$

Radiation $\operatorname{CuK} \alpha(\lambda=1.54184)$

$2 \Theta$ range for data collection $/{ }^{\circ} 6.33$ to 134.13

Index ranges $\quad-17 \leqslant \mathrm{~h} \leqslant 17,-17 \leqslant \mathrm{k} \leqslant 13,-22 \leqslant 1 \leqslant 19$

Reflections collected 14192 
Independent reflections $\quad 6861[$ Rint $=0.0609$, Rsigma $=0.0489]$

Data/restraints/parameters 6861/2/405

Goodness-of-fit on F2 1.061

Final $\mathrm{R}$ indexes $[\mathrm{I}>=2 \sigma(\mathrm{I})] \quad \mathrm{R} 1=0.0836, \mathrm{wR} 2=0.2442$

Final R indexes [all data] $\mathrm{R} 1=0.0951, \mathrm{wR} 2=0.2617$

Largest diff. peak/hole / e $\AA-3 \quad 2.83 /-0.38$

Table 6-2 Bond Lengths for 13.

\begin{tabular}{llllll}
\hline Atom & Atom & Length/A & Atom & Atom & Length/A \\
\hline $\mathrm{C} 1$ & $\mathrm{C} 2$ & $1.380(6)$ & $\mathrm{C} 20$ & $\mathrm{~N} 2$ & $1.374(5)$ \\
$\mathrm{C} 1$ & $\mathrm{C} 6$ & $1.405(6)$ & $\mathrm{C} 20$ & $\mathrm{P} 1$ & $1.794(4)$ \\
$\mathrm{C} 1$ & $\mathrm{P} 1$ & $1.797(4)$ & $\mathrm{C} 21$ & $\mathrm{C} 22$ & $1.411(5)$ \\
$\mathrm{C} 2$ & $\mathrm{C} 3$ & $1.380(7)$ & $\mathrm{C} 21$ & $\mathrm{C} 26$ & $1.417(5)$ \\
$\mathrm{C} 3$ & $\mathrm{C} 4$ & $1.380(9)$ & $\mathrm{C} 21$ & $\mathrm{P} 2$ & $1.756(4)$ \\
$\mathrm{C} 4$ & $\mathrm{C} 5$ & $1.377(8)$ & $\mathrm{C} 22$ & $\mathrm{C} 23$ & $1.404(6)$ \\
$\mathrm{C} 5$ & $\mathrm{C} 6$ & $1.371(7)$ & $\mathrm{C} 22$ & $\mathrm{~N} 2$ & $1.374(5)$ \\
$\mathrm{C} 7$ & $\mathrm{C} 8$ & $1.391(5)$ & $\mathrm{C} 23$ & $\mathrm{C} 24$ & $1.345(7)$ \\
$\mathrm{C} 7$ & $\mathrm{C} 12$ & $1.375(6)$ & $\mathrm{C} 24$ & $\mathrm{C} 25$ & $1.390(7)$ \\
$\mathrm{C} 7$ & $\mathrm{P} 1$ & $1.789(4)$ & $\mathrm{C} 25$ & $\mathrm{C} 26$ & $1.362(6)$ \\
$\mathrm{C} 8$ & $\mathrm{C} 9$ & $1.386(6)$ & $\mathrm{C} 27$ & $\mathrm{C} 28$ & $1.395(6)$ \\
$\mathrm{C} 9$ & $\mathrm{C} 10$ & $1.382(8)$ & $\mathrm{C} 27$ & $\mathrm{C} 32$ & $1.394(5)$ \\
$\mathrm{C} 10$ & $\mathrm{C} 11$ & $1.361(8)$ & $\mathrm{C} 27$ & $\mathrm{P} 2$ & $1.786(4)$ \\
\hline
\end{tabular}




\begin{tabular}{llllll}
\hline $\mathrm{C} 11$ & $\mathrm{C} 12$ & $1.371(7)$ & $\mathrm{C} 28$ & $\mathrm{C} 29$ & $1.350(7)$ \\
$\mathrm{C} 13$ & $\mathrm{C} 14$ & $1.418(5)$ & $\mathrm{C} 29$ & $\mathrm{C} 30$ & $1.386(9)$ \\
$\mathrm{C} 13$ & $\mathrm{C} 18$ & $1.396(5)$ & $\mathrm{C} 30$ & $\mathrm{C} 31$ & $1.387(8)$ \\
$\mathrm{C} 13$ & $\mathrm{P} 1$ & $1.775(4)$ & $\mathrm{C} 31$ & $\mathrm{C} 32$ & $1.377(7)$ \\
$\mathrm{C} 14$ & $\mathrm{C} 15$ & $1.367(7)$ & $\mathrm{C} 33$ & $\mathrm{C} 34$ & $1.398(6)$ \\
$\mathrm{C} 15$ & $\mathrm{C} 16$ & $1.395(7)$ & $\mathrm{C} 33$ & $\mathrm{C} 38$ & $1.399(6)$ \\
$\mathrm{C} 16$ & $\mathrm{C} 17$ & $1.376(6)$ & $\mathrm{C} 33$ & $\mathrm{P} 2$ & $1.798(4)$ \\
$\mathrm{C} 17$ & $\mathrm{C} 18$ & $1.403(6)$ & $\mathrm{C} 34$ & $\mathrm{C} 35$ & $1.401(7)$ \\
$\mathrm{C} 18$ & $\mathrm{~N} 1$ & $1.376(5)$ & $\mathrm{C} 35$ & $\mathrm{C} 36$ & $1.351(9)$ \\
$\mathrm{C} 19$ & $\mathrm{C} 20$ & $1.372(5)$ & $\mathrm{C} 36$ & $\mathrm{C} 37$ & $1.363(9)$ \\
$\mathrm{C} 19$ & $\mathrm{~N} 1$ & $1.377(5)$ & $\mathrm{C} 37$ & $\mathrm{C} 38$ & $1.375(7)$ \\
$\mathrm{C} 19$ & $\mathrm{P} 2$ & $1.801(4)$ & & & \\
\hline
\end{tabular}

Table 6-3 Bond Angles for 13.

\begin{tabular}{llllllll}
\hline \multicolumn{2}{c}{ Atom Atom Atom } & Angle $^{\circ}$ & Atom Atom Atom & Angle $^{\circ}$ \\
\hline C2 & C1 & C6 & $119.3(4)$ & N2 & C22 & C21 & $122.9(3)$ \\
C2 & C1 & P1 & $121.4(3)$ & N2 & C22 & C23 & $119.9(3)$ \\
C6 & C1 & P1 & $119.2(3)$ & C24 & C23 & C22 & $121.5(4)$ \\
C3 & C2 & C1 & $119.8(5)$ & C23 & C24 & C25 & $121.7(4)$ \\
C4 & C3 & C2 & $120.7(5)$ & C26 & C25 & C24 & $119.3(4)$ \\
C5 & C4 & C3 & $119.7(4)$ & C25 & C26 & C21 & $120.2(4)$ \\
\hline
\end{tabular}




\begin{tabular}{|c|c|c|c|c|c|c|c|}
\hline C6 & $\mathrm{C} 5$ & $\mathrm{C} 4$ & $120.3(5)$ & $\mathrm{C} 28$ & $\mathrm{C} 27$ & $\mathrm{P} 2$ & $119.2(3)$ \\
\hline C5 & C6 & $\mathrm{C} 1$ & $120.1(4)$ & C32 & $\mathrm{C} 27$ & $\mathrm{C} 28$ & $120.2(4)$ \\
\hline $\mathrm{C} 8$ & $\mathrm{C} 7$ & $\mathrm{P} 1$ & $121.7(3)$ & C32 & $\mathrm{C} 27$ & $\mathrm{P} 2$ & $119.9(3)$ \\
\hline $\mathrm{C} 12$ & $\mathrm{C} 7$ & $\mathrm{C} 8$ & $119.7(4)$ & $\mathrm{C} 29$ & $\mathrm{C} 28$ & $\mathrm{C} 27$ & $120.9(4)$ \\
\hline $\mathrm{C} 12$ & $\mathrm{C} 7$ & $\mathrm{P} 1$ & $118.6(3)$ & $\mathrm{C} 28$ & $\mathrm{C} 29$ & $\mathrm{C} 30$ & $119.6(5)$ \\
\hline C9 & $\mathrm{C} 8$ & $\mathrm{C} 7$ & $119.2(4)$ & $\mathrm{C} 29$ & $\mathrm{C} 30$ & $\mathrm{C} 31$ & $120.0(4)$ \\
\hline $\mathrm{C} 10$ & $\mathrm{C} 9$ & $\mathrm{C} 8$ & $120.5(4)$ & C32 & C 31 & $\mathrm{C} 30$ & $121.0(5)$ \\
\hline $\mathrm{C} 11$ & $\mathrm{C} 10$ & C9 & 119.1(4) & C31 & $\mathrm{C} 32$ & $\mathrm{C} 27$ & $118.3(5)$ \\
\hline C10 & $\mathrm{C} 11$ & $\mathrm{C} 12$ & $121.5(5)$ & C34 & $\mathrm{C} 33$ & C38 & $120.1(4)$ \\
\hline $\mathrm{C} 11$ & $\mathrm{C} 12$ & $\mathrm{C} 7$ & $119.9(4)$ & C34 & $\mathrm{C} 33$ & $\mathrm{P} 2$ & $120.6(3)$ \\
\hline C14 & $\mathrm{C} 13$ & $\mathrm{P} 1$ & $118.5(3)$ & C38 & $\mathrm{C} 33$ & $\mathrm{P} 2$ & 119.1(3) \\
\hline C18 & $\mathrm{C} 13$ & $\mathrm{C} 14$ & $119.8(4)$ & C33 & C34 & $\mathrm{C} 35$ & $117.4(5)$ \\
\hline C18 & $\mathrm{C} 13$ & $\mathrm{P} 1$ & $121.6(3)$ & C36 & $\mathrm{C} 35$ & $\mathrm{C} 34$ & $121.9(5)$ \\
\hline C15 & C14 & $\mathrm{C} 13$ & $120.0(4)$ & $\mathrm{C} 35$ & C36 & $\mathrm{C} 37$ & $120.4(5)$ \\
\hline C14 & C15 & $\mathrm{C} 16$ & 119.7(4) & C36 & C 37 & C38 & $120.4(5)$ \\
\hline C17 & C16 & $\mathrm{C} 15$ & $121.4(4)$ & C37 & C 38 & $\mathrm{C} 33$ & $119.8(4)$ \\
\hline C16 & $\mathrm{C} 17$ & $\mathrm{C} 18$ & 119.6(4) & $\mathrm{C} 18$ & N1 & C19 & $125.5(3)$ \\
\hline C13 & C18 & $\mathrm{C} 17$ & $119.5(4)$ & $\mathrm{C} 20$ & $\mathrm{~N} 2$ & $\mathrm{C} 22$ & $125.7(3)$ \\
\hline N1 & $\mathrm{C} 18$ & $\mathrm{C} 13$ & $122.0(3)$ & $\mathrm{C} 7$ & $\mathrm{P} 1$ & $\mathrm{C} 1$ & $110.50(17)$ \\
\hline N1 & C18 & $\mathrm{C} 17$ & $118.5(3)$ & $\mathrm{C} 7$ & $\mathrm{P} 1$ & $\mathrm{C} 20$ & $110.99(17)$ \\
\hline $\mathrm{C} 20$ & C19 & N1 & $126.6(3)$ & $\mathrm{C} 13$ & $\mathrm{P} 1$ & $\mathrm{C} 1$ & $112.63(18)$ \\
\hline
\end{tabular}




\begin{tabular}{llllllll}
\hline $\mathrm{C} 20$ & $\mathrm{C} 19$ & $\mathrm{P} 2$ & $119.1(3)$ & $\mathrm{C} 13$ & $\mathrm{P} 1$ & $\mathrm{C} 7$ & $109.40(17)$ \\
$\mathrm{N} 1$ & $\mathrm{C} 19$ & $\mathrm{P} 2$ & $114.2(2)$ & $\mathrm{C} 13$ & $\mathrm{P} 1$ & $\mathrm{C} 20$ & $103.35(17)$ \\
$\mathrm{C} 19$ & $\mathrm{C} 20$ & $\mathrm{~N} 2$ & $126.1(3)$ & $\mathrm{C} 20$ & $\mathrm{P} 1$ & $\mathrm{C} 1$ & $109.79(17)$ \\
$\mathrm{C} 19$ & $\mathrm{C} 20$ & $\mathrm{P} 1$ & $118.3(3)$ & $\mathrm{C} 21$ & $\mathrm{P} 2$ & $\mathrm{C} 19$ & $104.03(17)$ \\
$\mathrm{N} 2$ & $\mathrm{C} 20$ & $\mathrm{P} 1$ & $115.6(2)$ & $\mathrm{C} 21$ & $\mathrm{P} 2$ & $\mathrm{C} 27$ & $109.92(17)$ \\
$\mathrm{C} 22$ & $\mathrm{C} 21$ & $\mathrm{C} 26$ & $120.0(3)$ & $\mathrm{C} 21$ & $\mathrm{P} 2$ & $\mathrm{C} 33$ & $110.79(17)$ \\
$\mathrm{C} 22$ & $\mathrm{C} 21$ & $\mathrm{P} 2$ & $121.4(3)$ & $\mathrm{C} 27$ & $\mathrm{P} 2$ & $\mathrm{C} 19$ & $110.45(17)$ \\
$\mathrm{C} 26$ & $\mathrm{C} 21$ & $\mathrm{P} 2$ & $118.6(3)$ & $\mathrm{C} 27$ & $\mathrm{P} 2$ & $\mathrm{C} 33$ & $111.50(17)$ \\
$\mathrm{C} 23$ & $\mathrm{C} 22$ & $\mathrm{C} 21$ & $117.2(4)$ & $\mathrm{C} 33$ & $\mathrm{P} 2$ & $\mathrm{C} 19$ & $109.91(17)$ \\
\hline
\end{tabular}

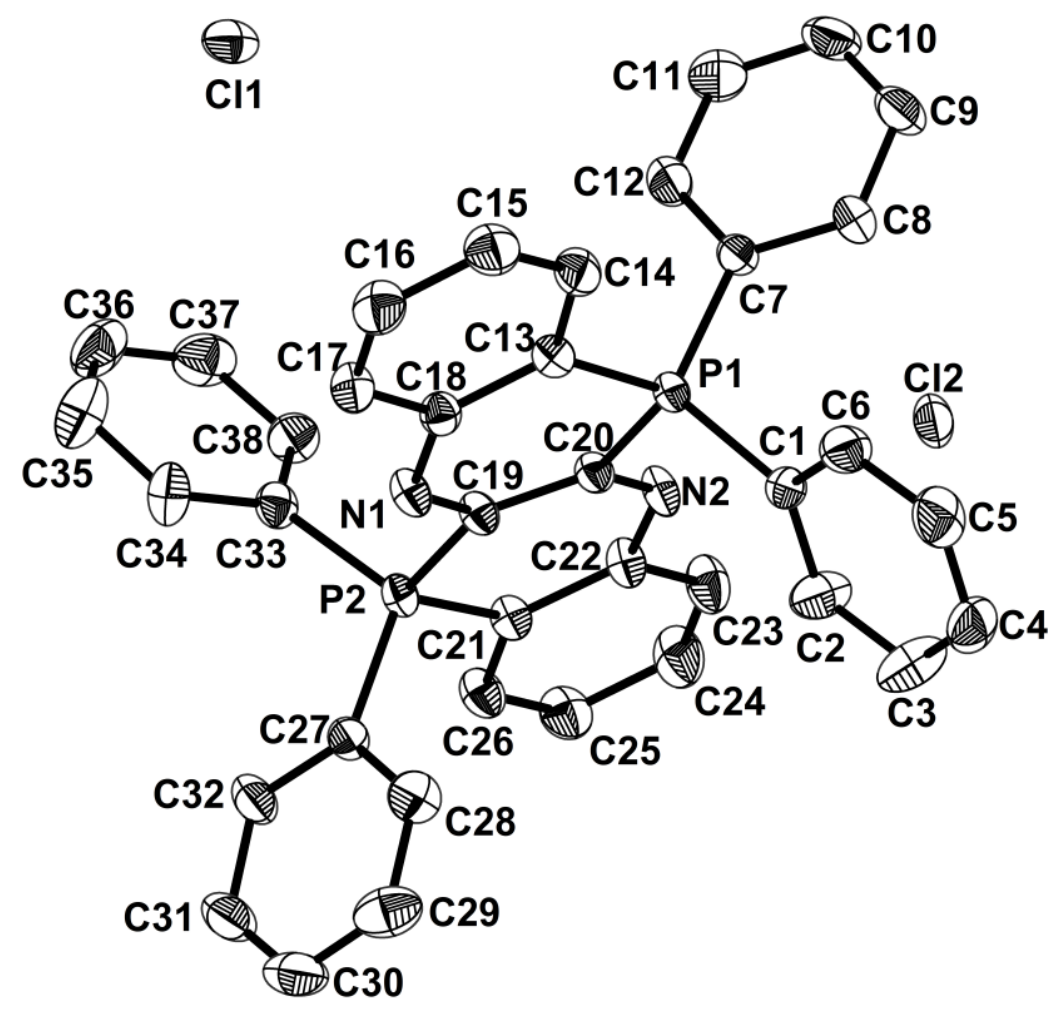

SFigure 6. ORTEP drawing of 13 with $30 \%$ probability thermal 
ellipsoids. Hydrogen atoms have been omitted for clarity.

5) Scanned ${ }^{31} \mathrm{P}$ NMR, ${ }^{1} \mathrm{H}$ NMR and ${ }^{13} \mathrm{C}$ NMR Spectra of All New Compounds 

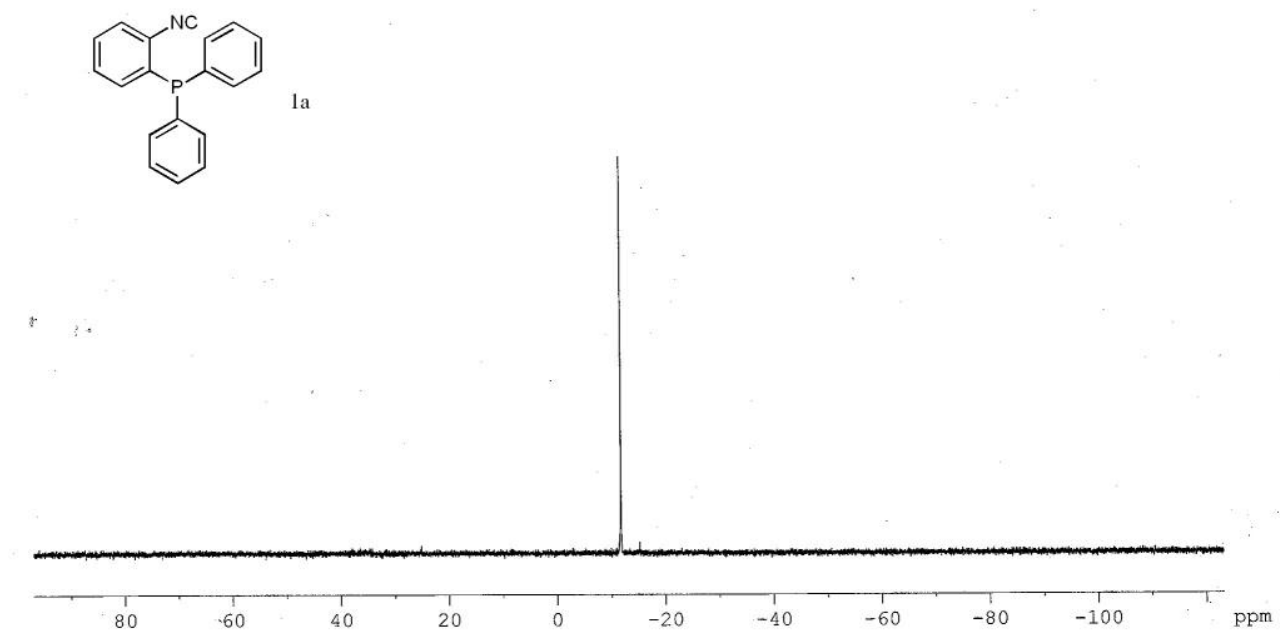

21j 286 CDCl3 PROTON

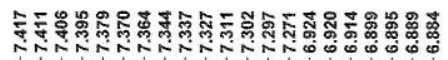

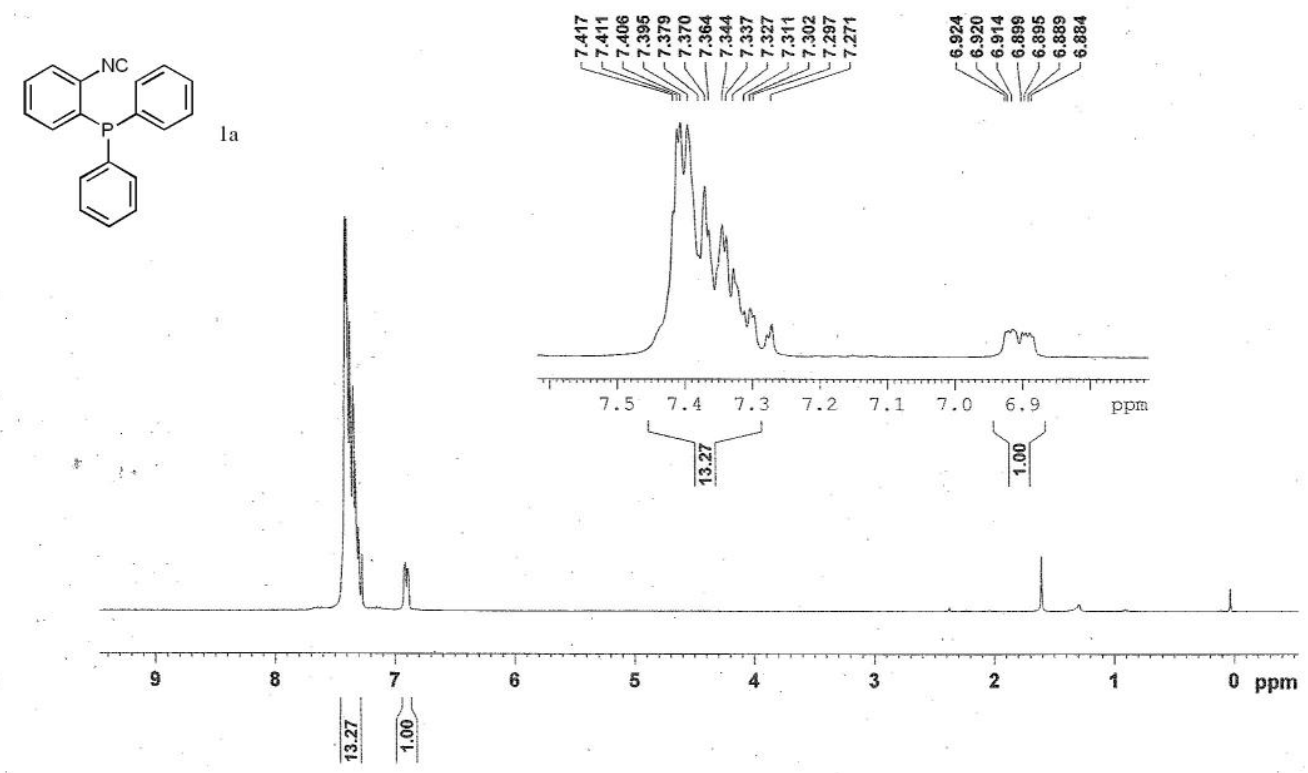




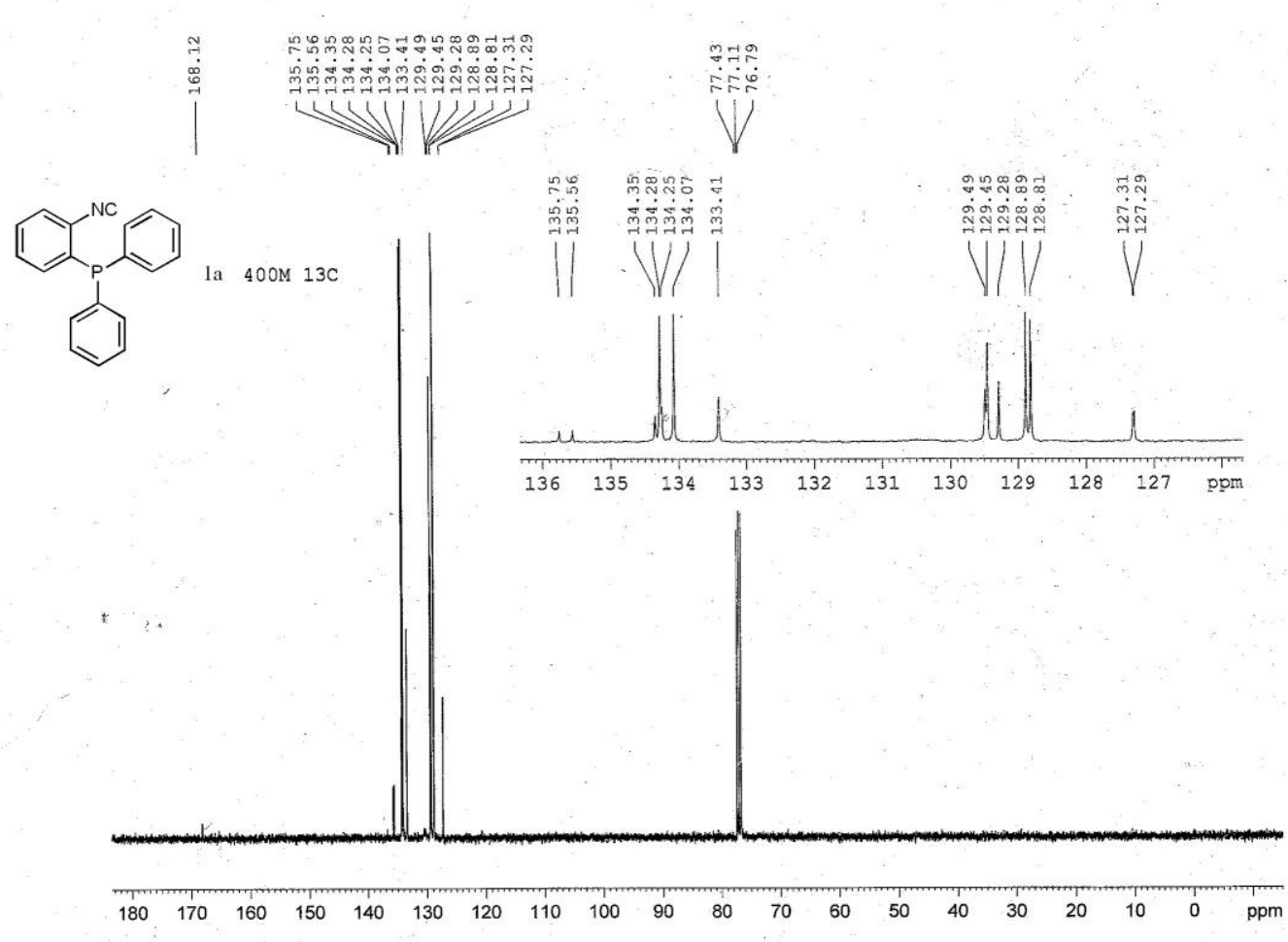

yuwenfei 189 P31CPD CDC13

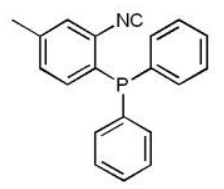

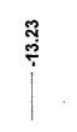

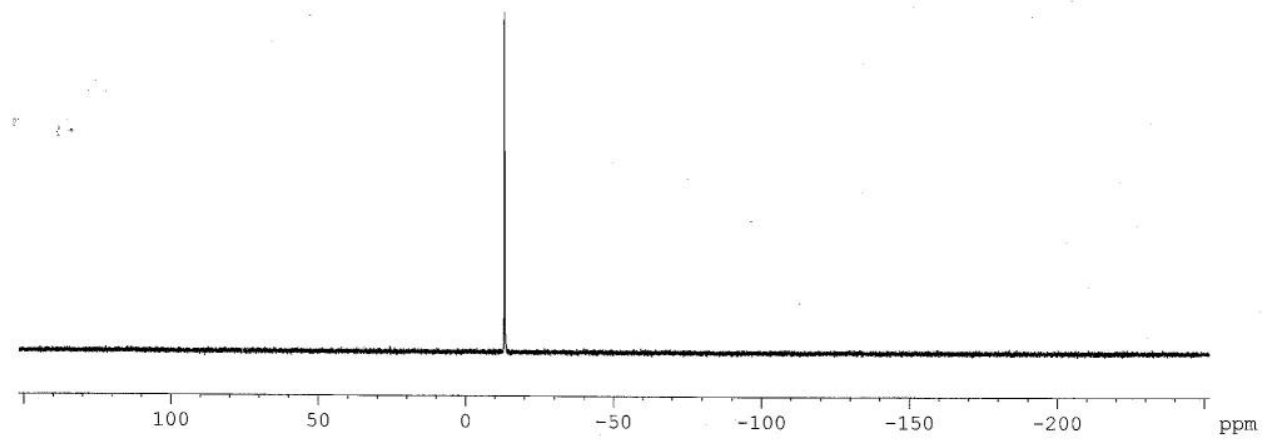




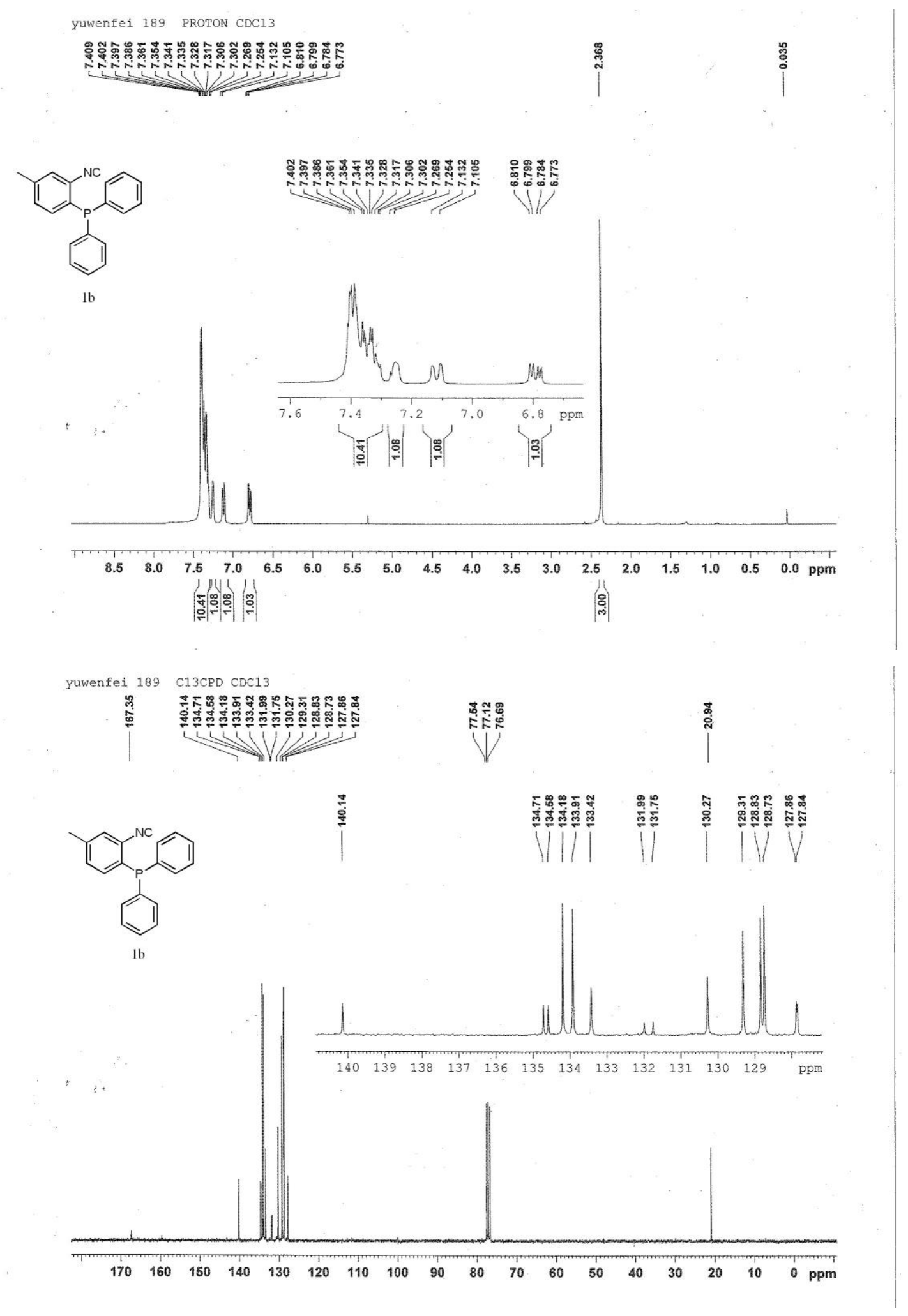




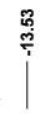

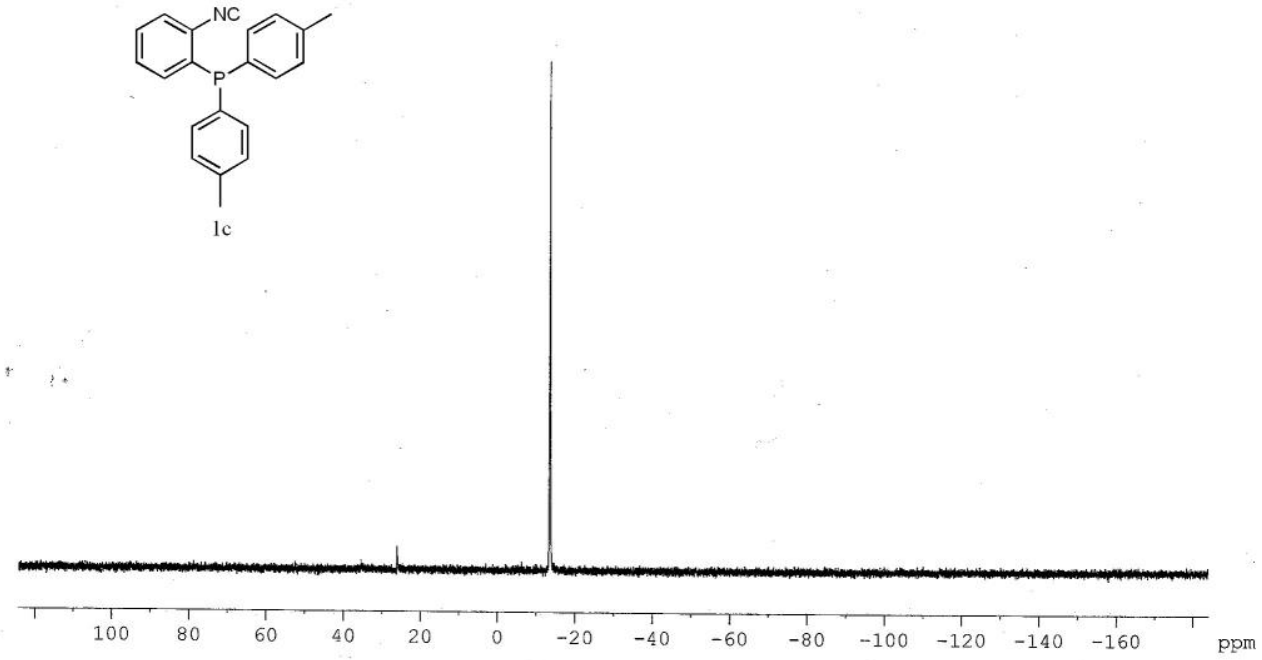

yuwenfei 223 PROTON CDC13

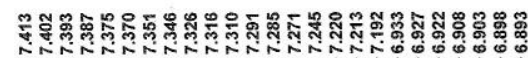
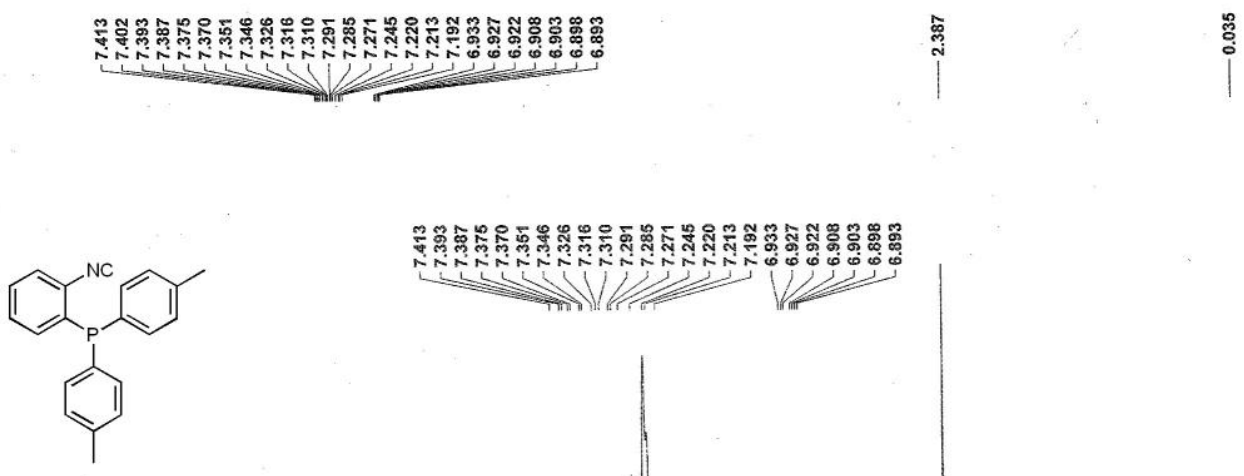

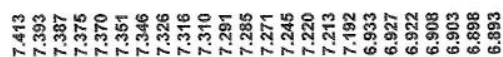

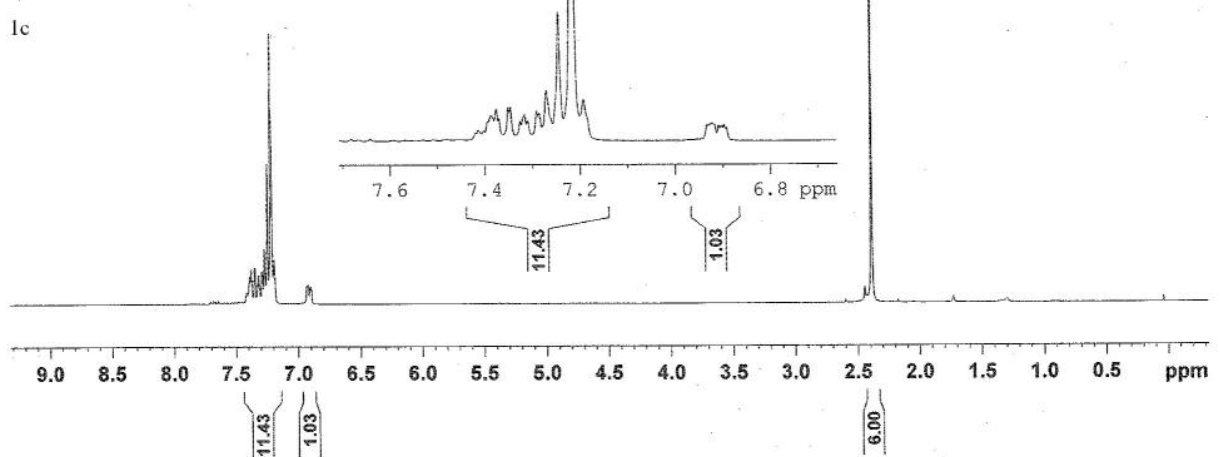



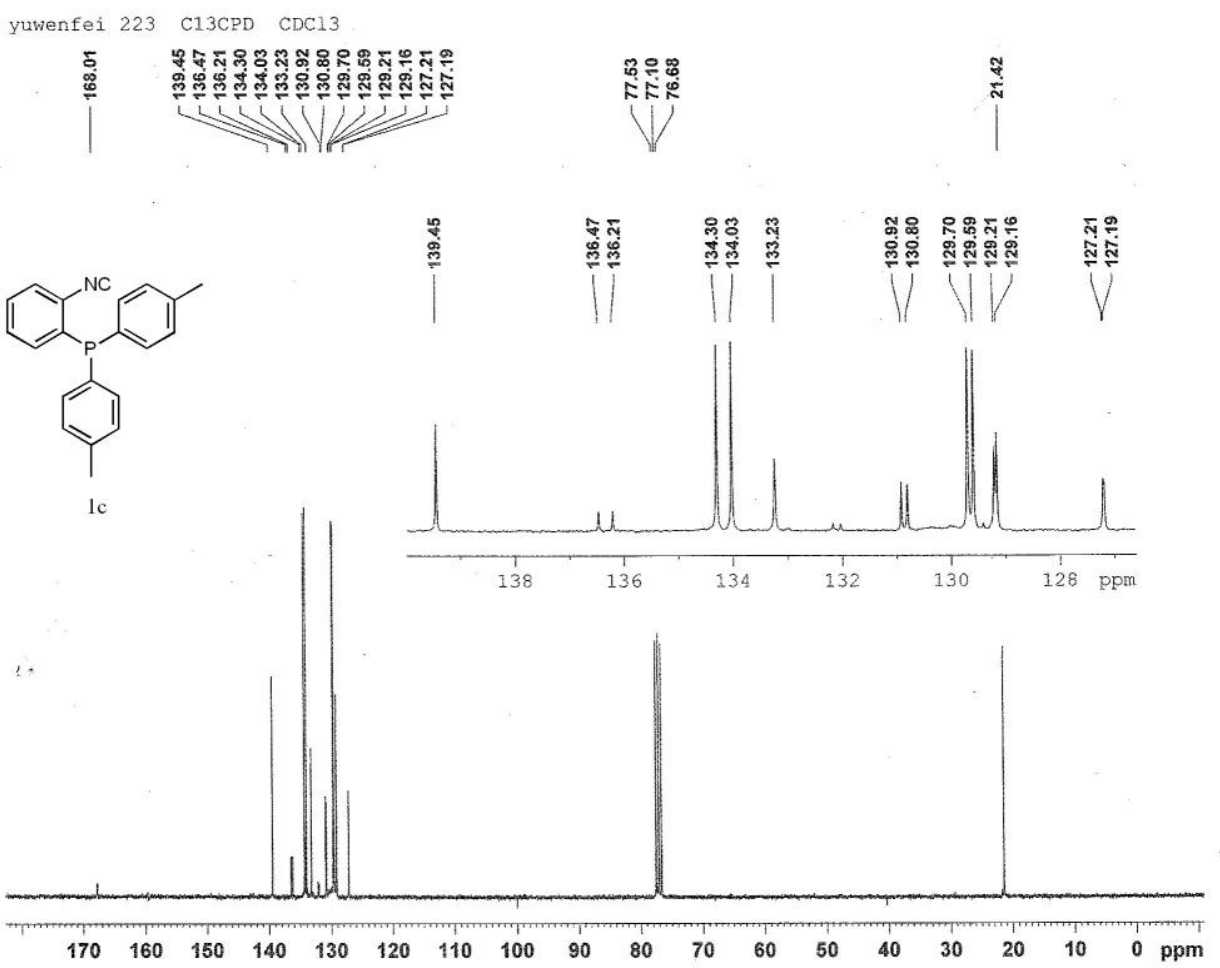

add Li $30 \mathrm{~min}$ P31CPD

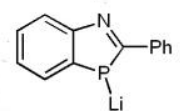


add Li $30 \mathrm{~min}$ P31CPD

| ָู

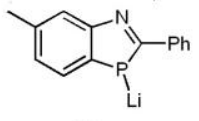

$3 b$

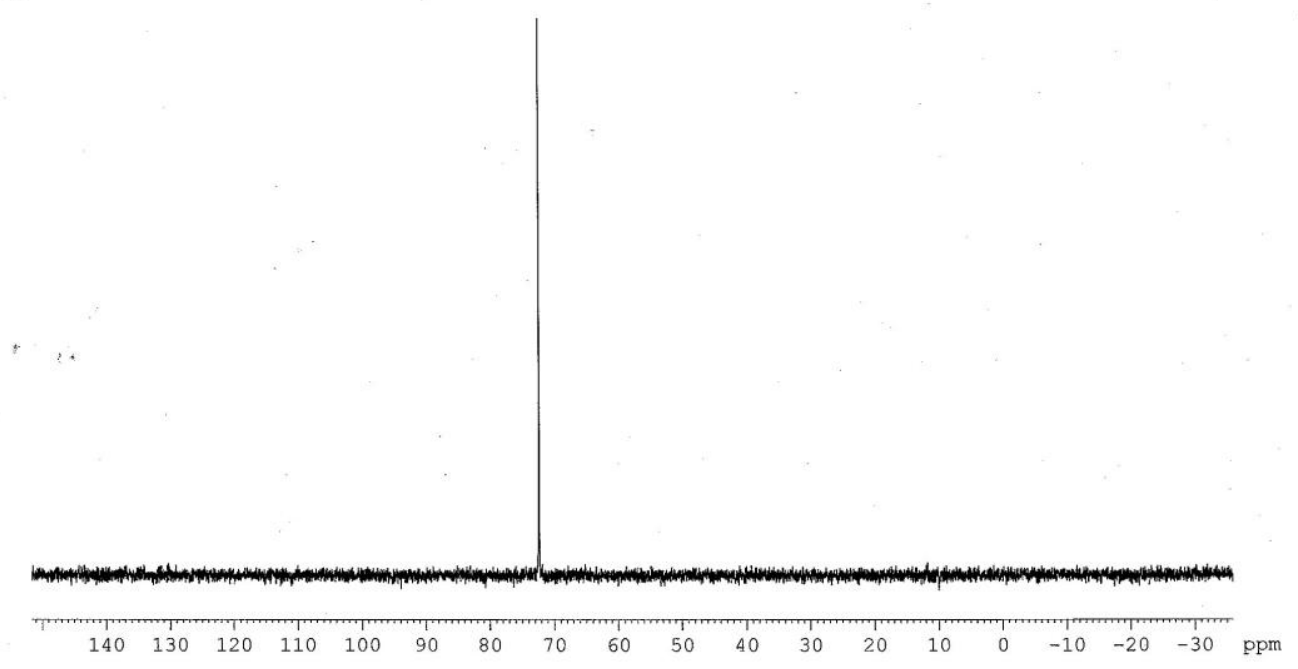

add Li $30 \mathrm{~min}$ P31CPD

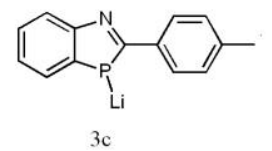

i

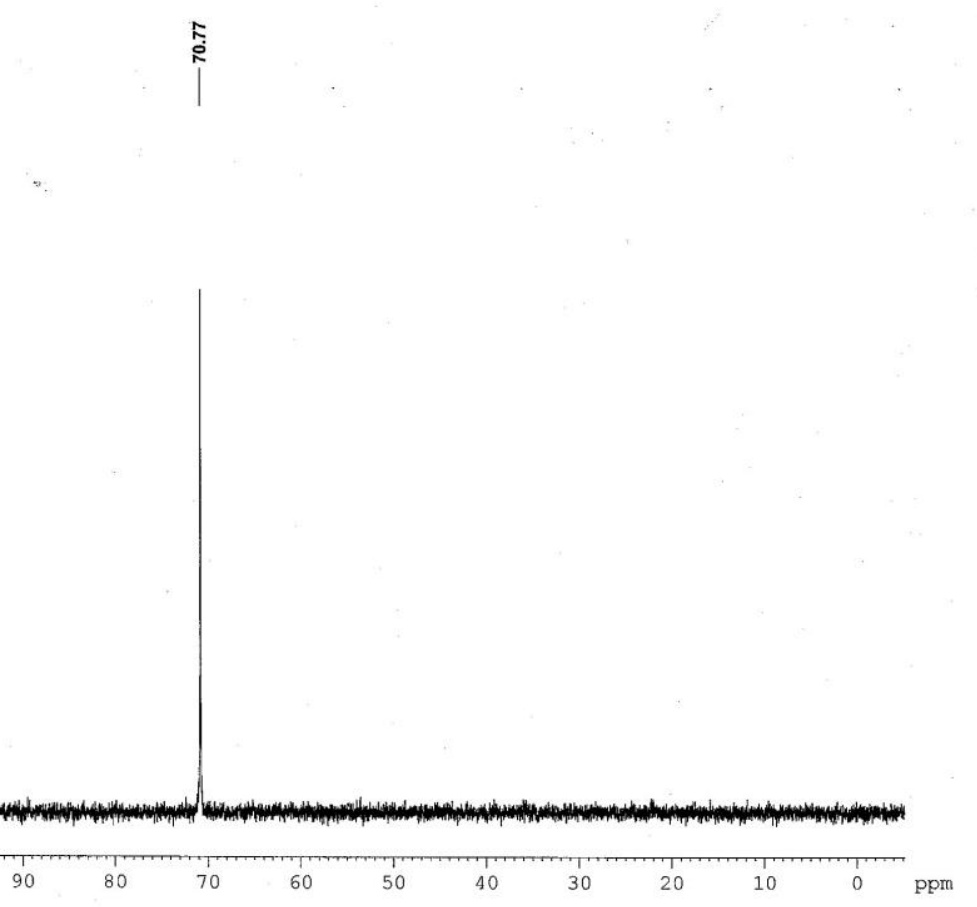



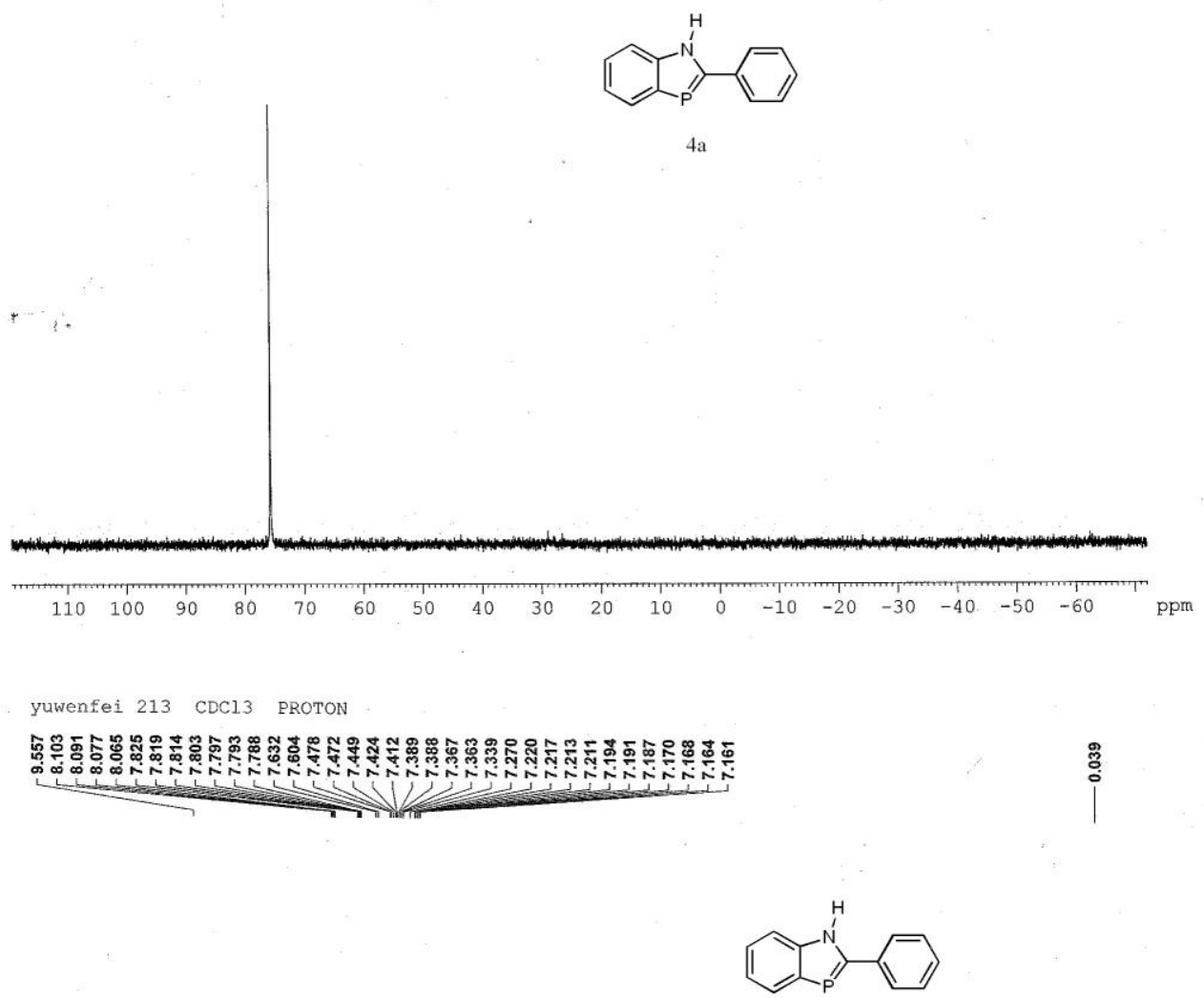

$4 \mathrm{a}$

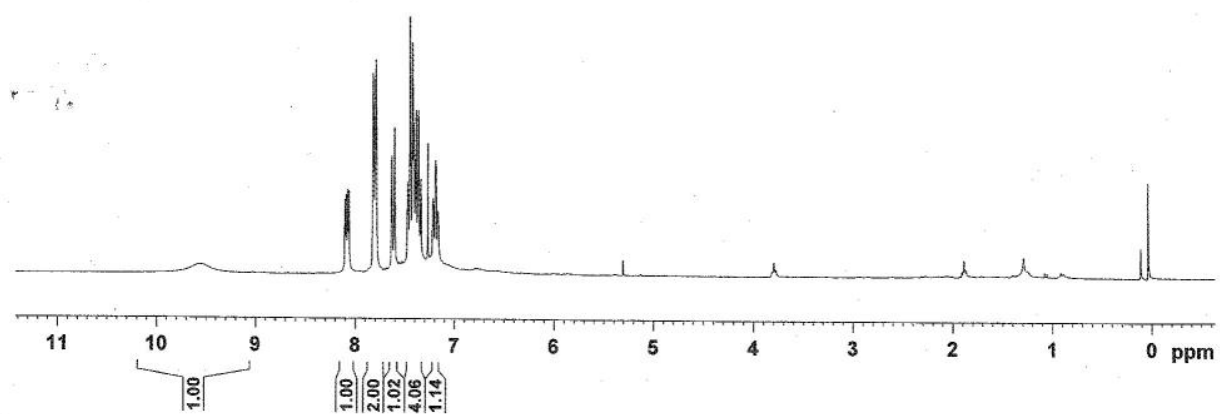




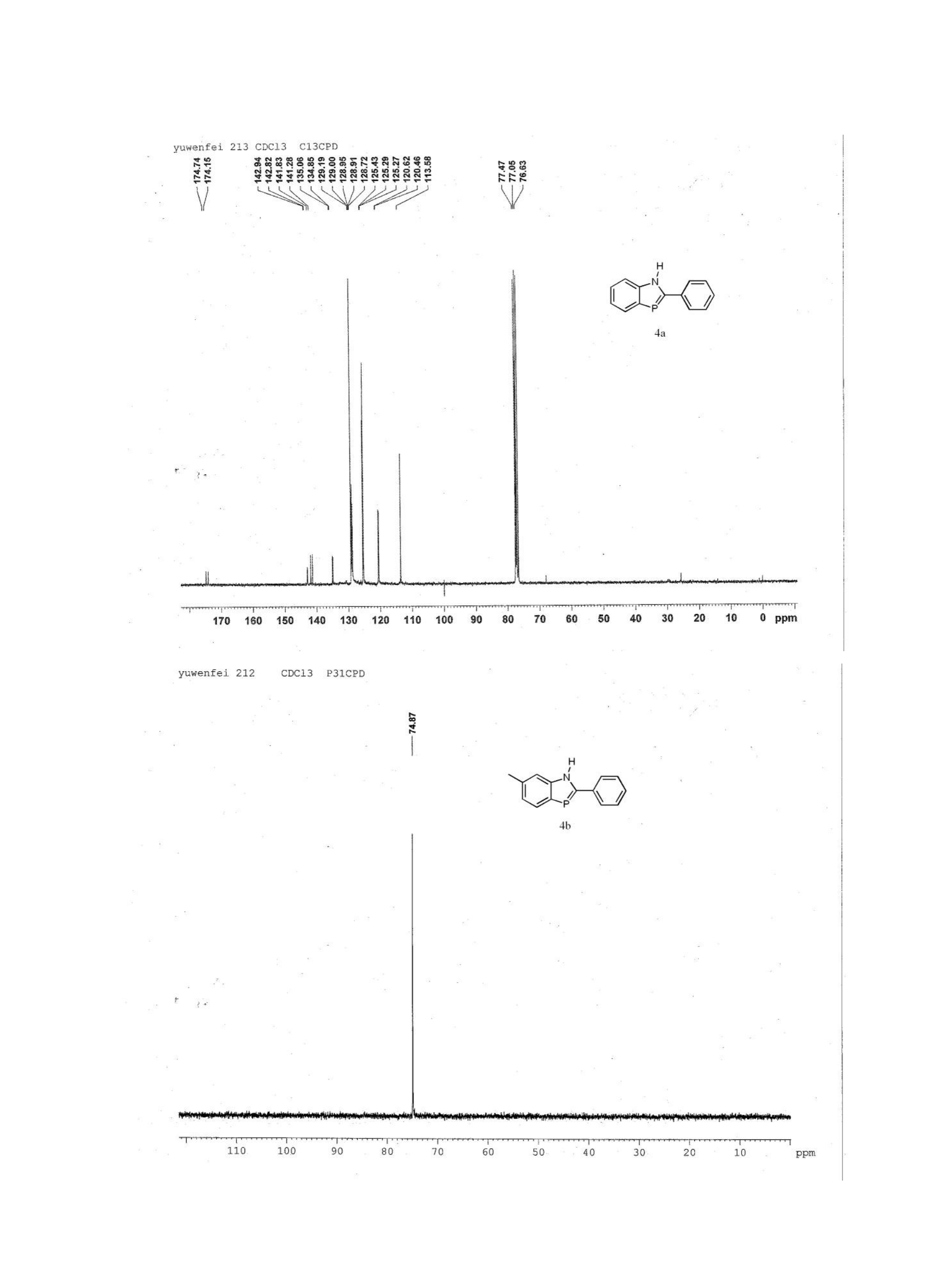




\section{苗}

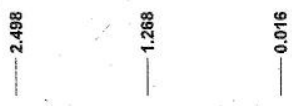

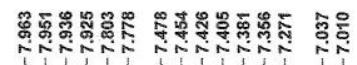

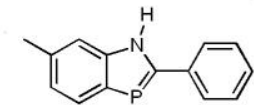

$4 \mathrm{~b}$

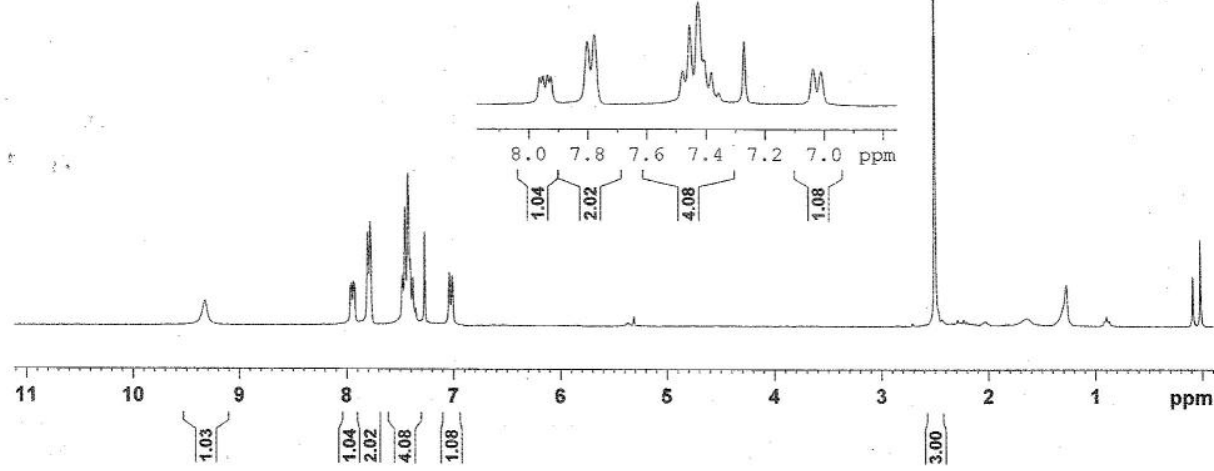

yuwenfei 212 CDC13 C13CPD

士

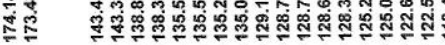

踶哭畐

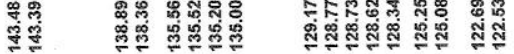

V VV W V V

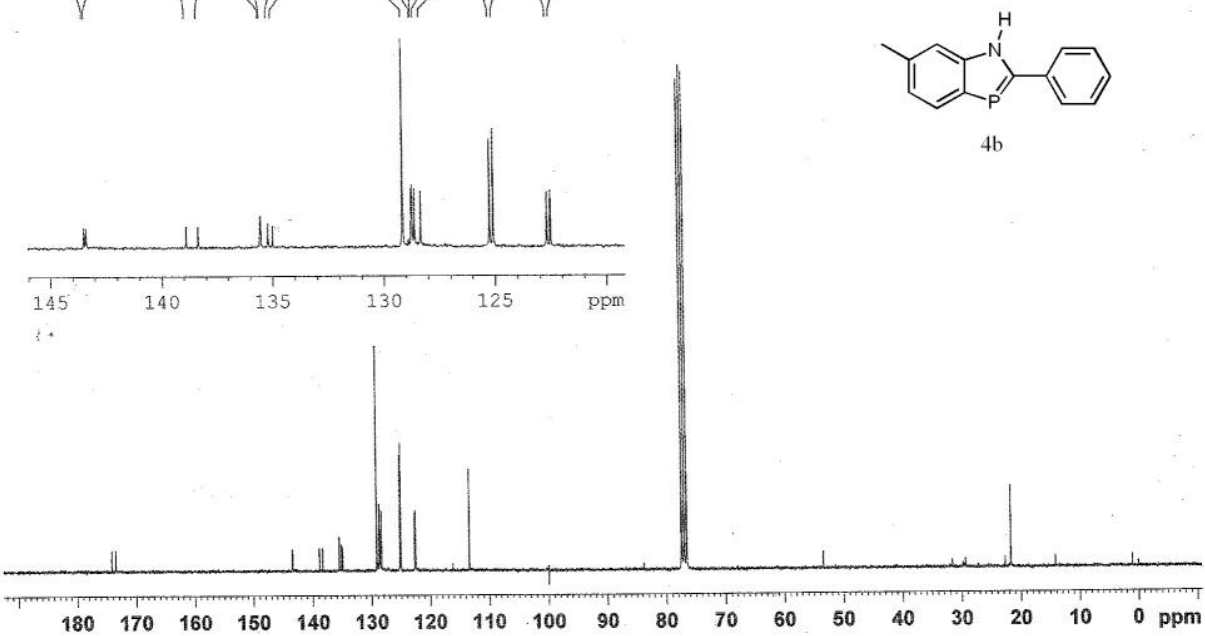




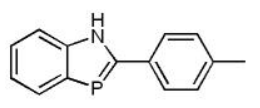

$4 \mathrm{c}$

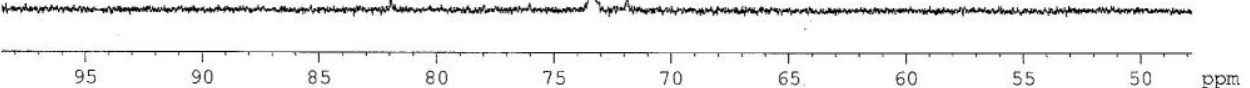

yuwenfei 261 RROTON CDC13

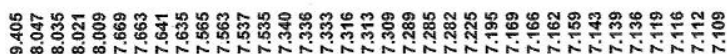

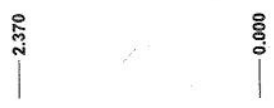

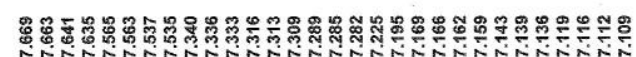
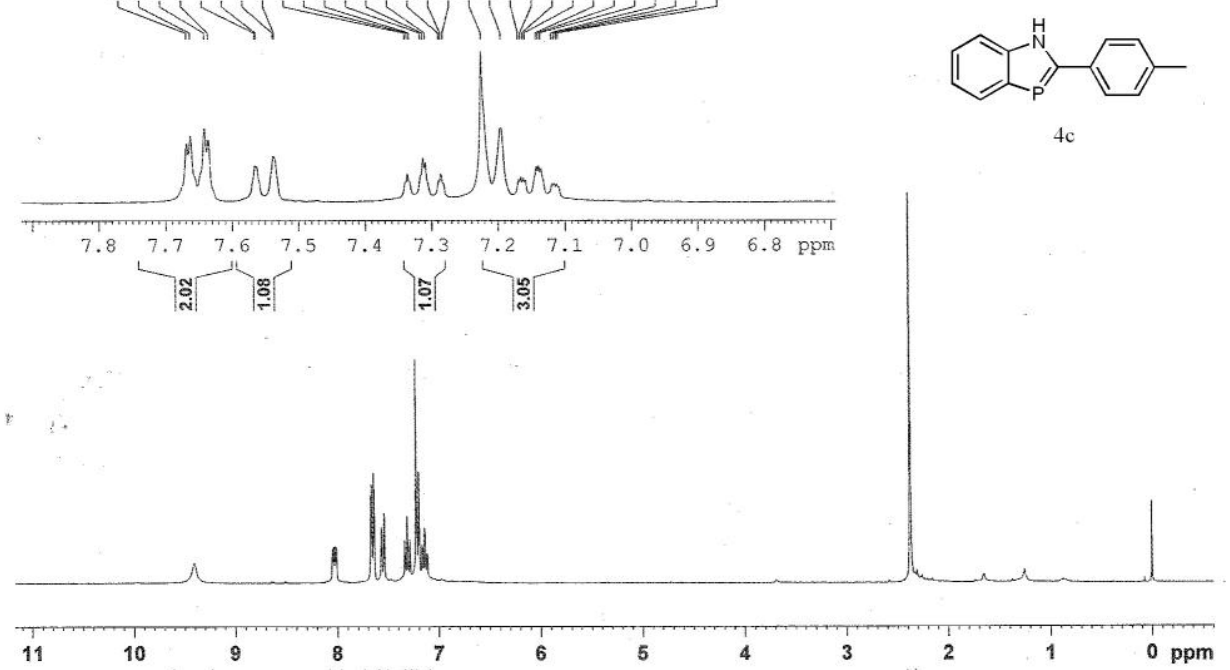

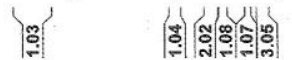

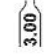




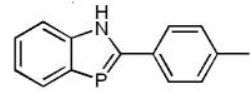

$4 \mathrm{c}$

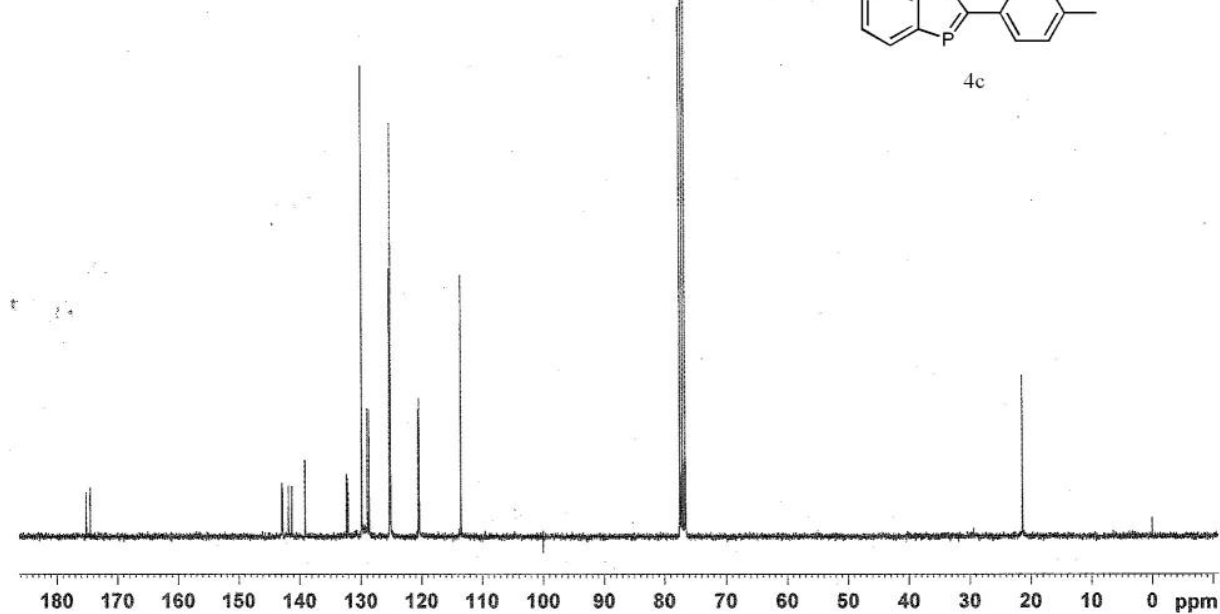

$\begin{array}{llll}\text { yuwenfei } & 187 & \mathrm{CDCl} 3 & \mathrm{P} 31 \mathrm{CPD}\end{array}$<smiles>S=P1(Cc2ccccc2)C(c2ccccc2)=Nc2ccccc21</smiles>

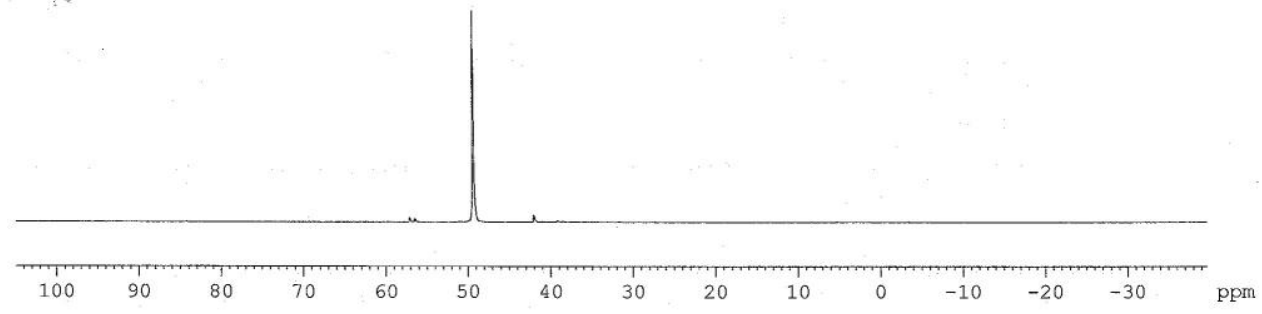




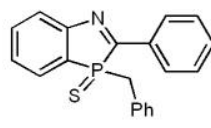
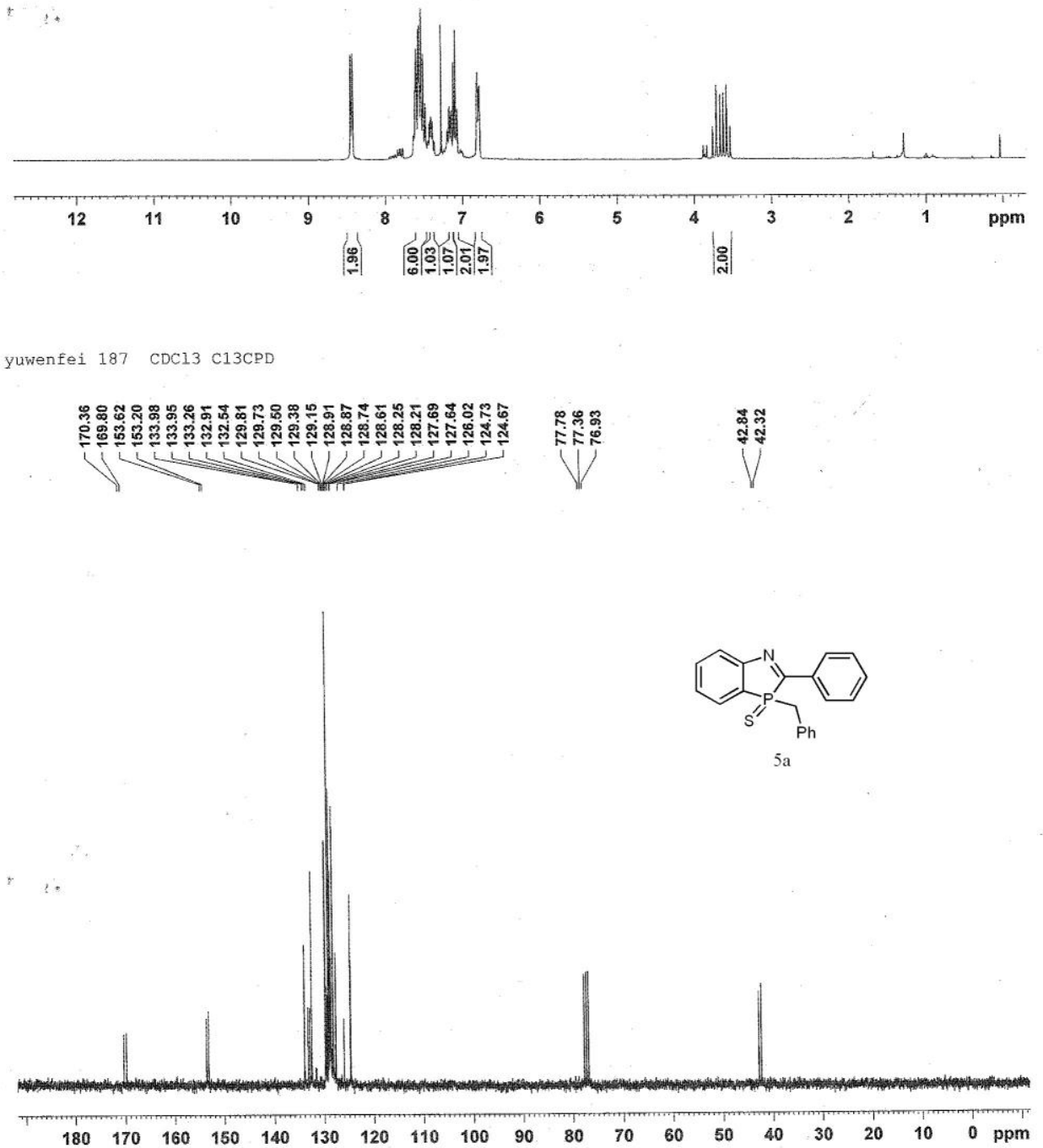
z1j $324 \quad$ P31CPD $\quad \mathrm{CDCl} 3$

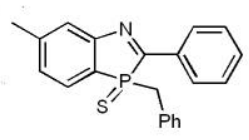

$5 \mathrm{~b}$

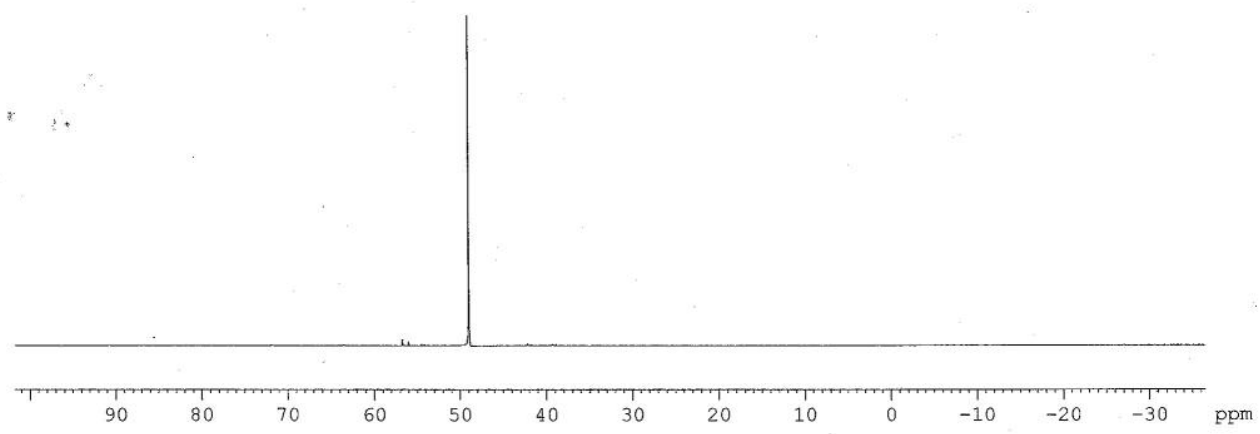

zlj $324 \quad \mathrm{CDCl3}$ PROTON

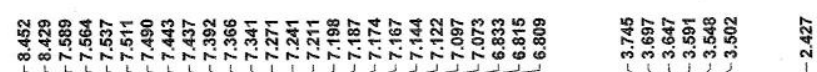

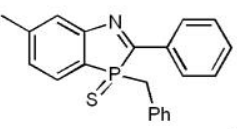

$5 b$

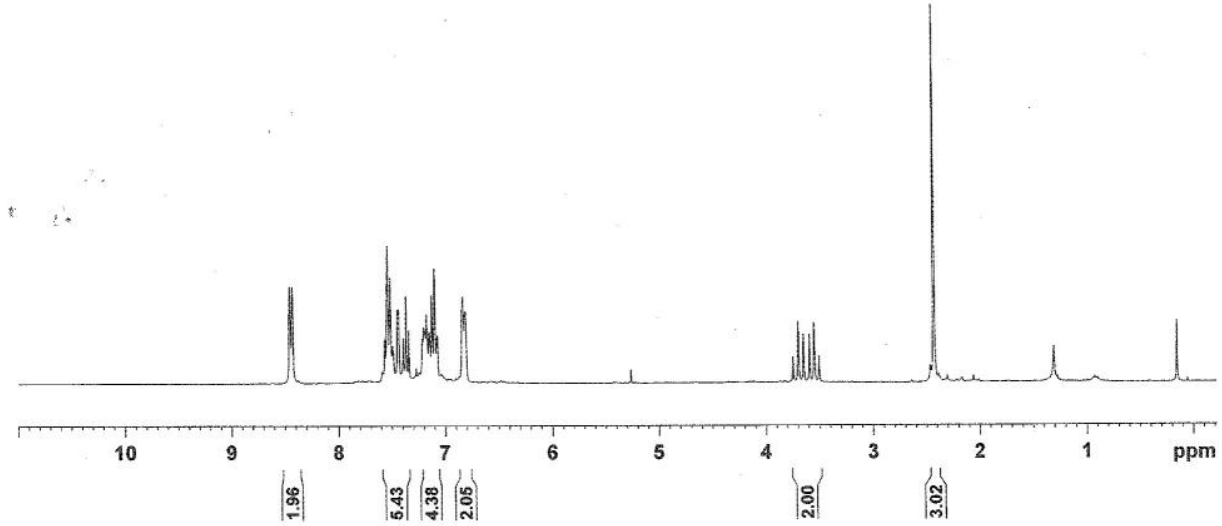


21j $324 \quad \operatorname{CDC13}$ C13CPD

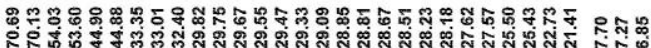

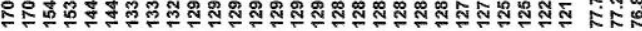

L

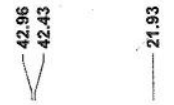

(N)

$5 b$

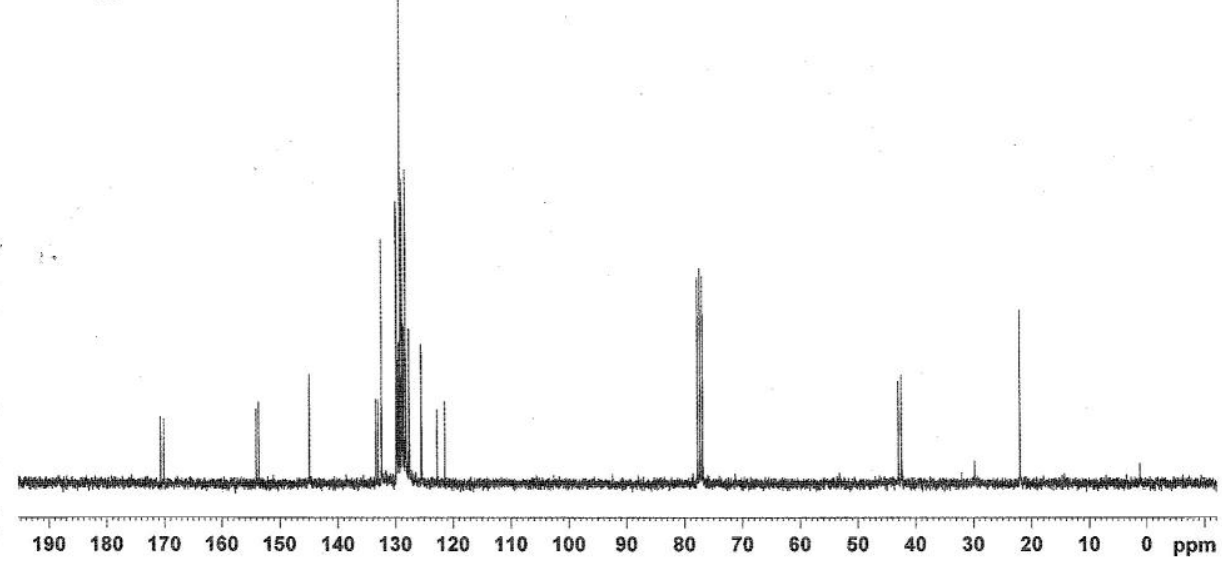

yuwenfei $216 \quad \mathrm{CDCl3} \quad$ P31CPD

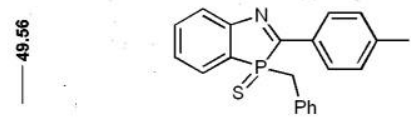

$5 \mathrm{c}$

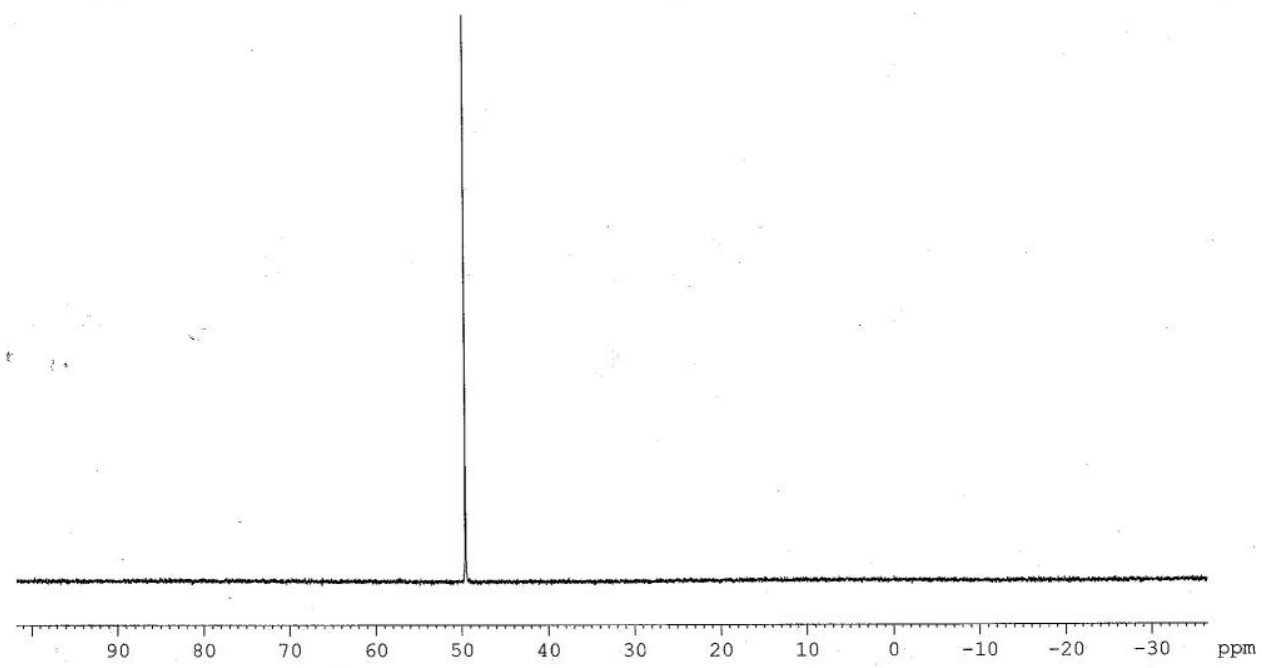



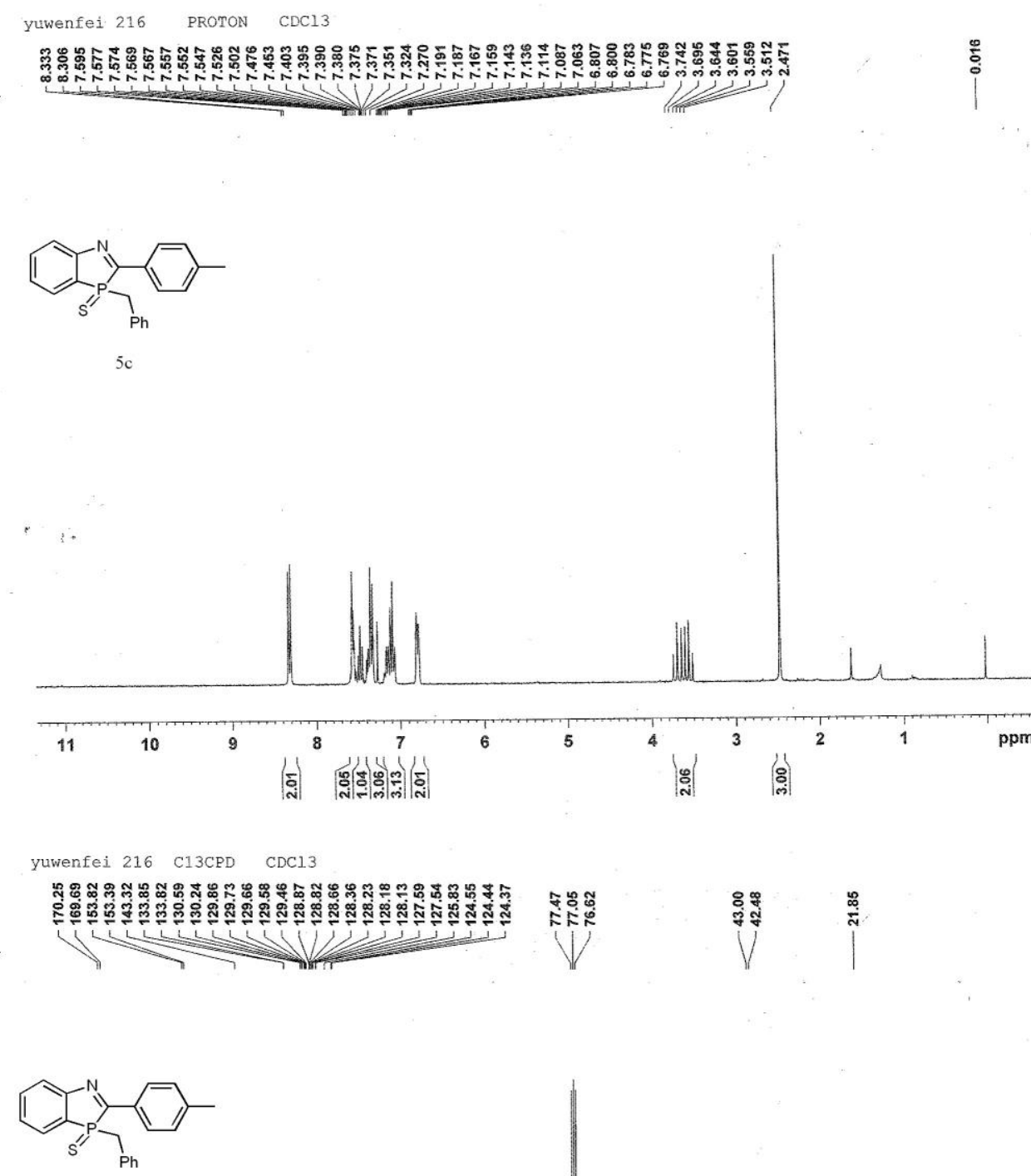

$5 \mathrm{c}$

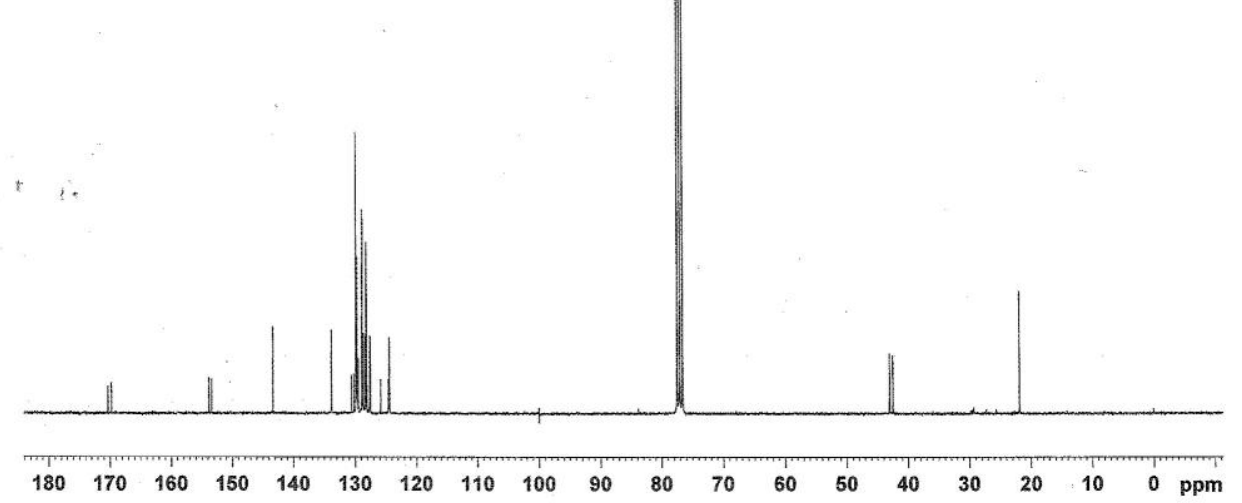



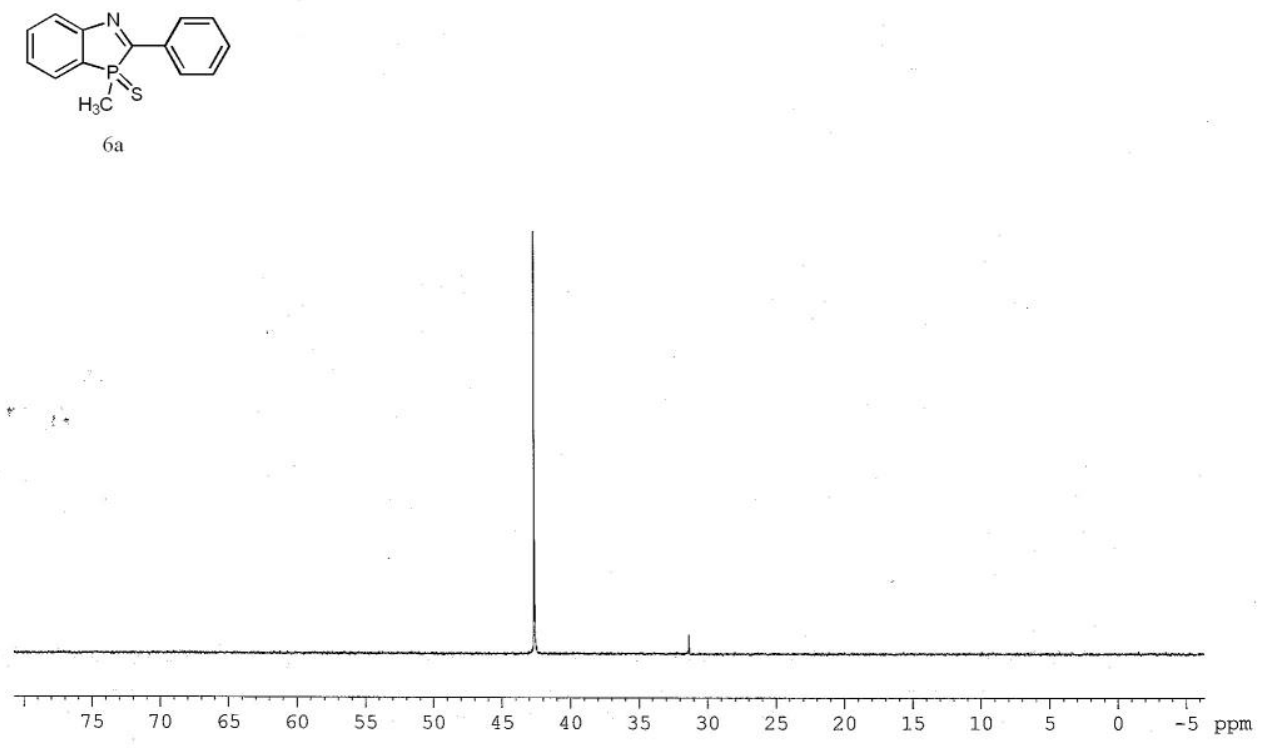

yuwenfei 256 PROTON CDC13

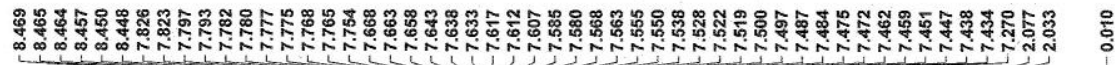

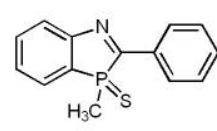

6a

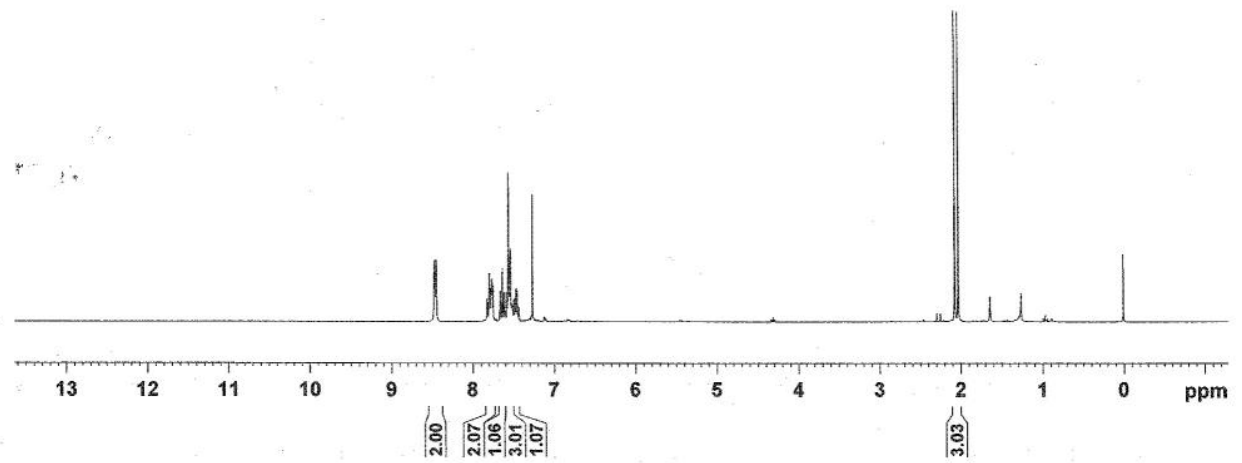


(N)

$6 \mathrm{a}$

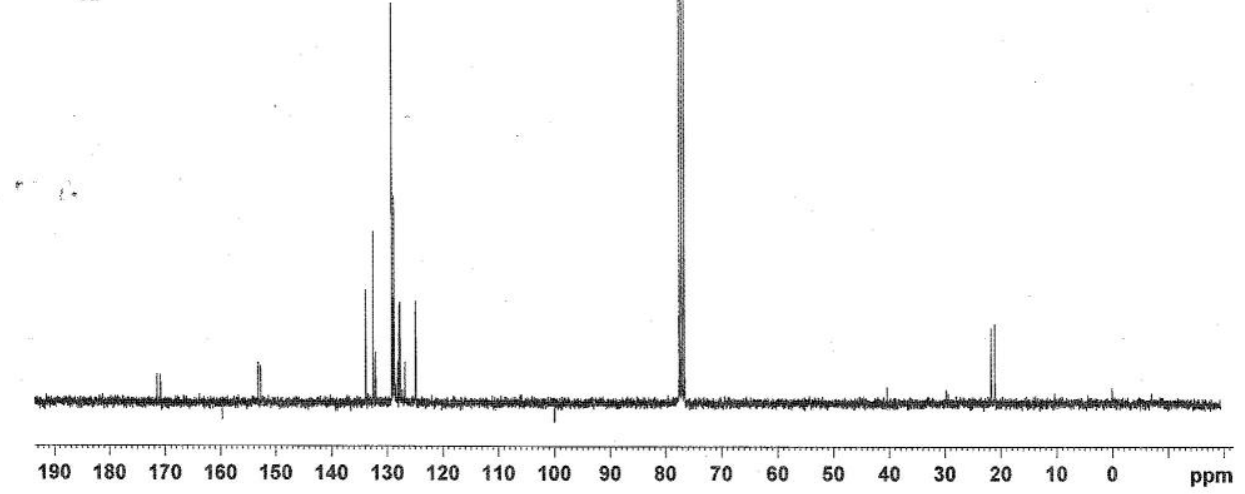

yuwenfei 222 P31CPD $\quad$ CDC13
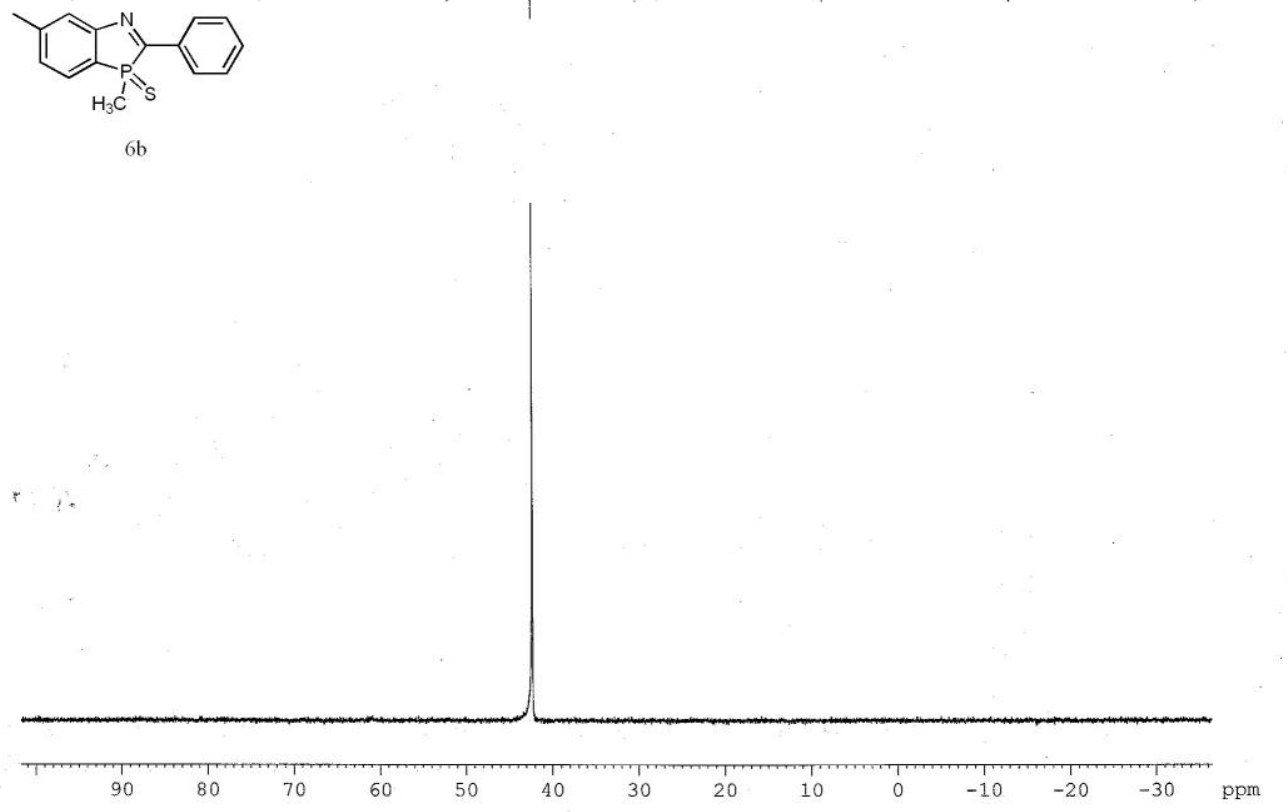

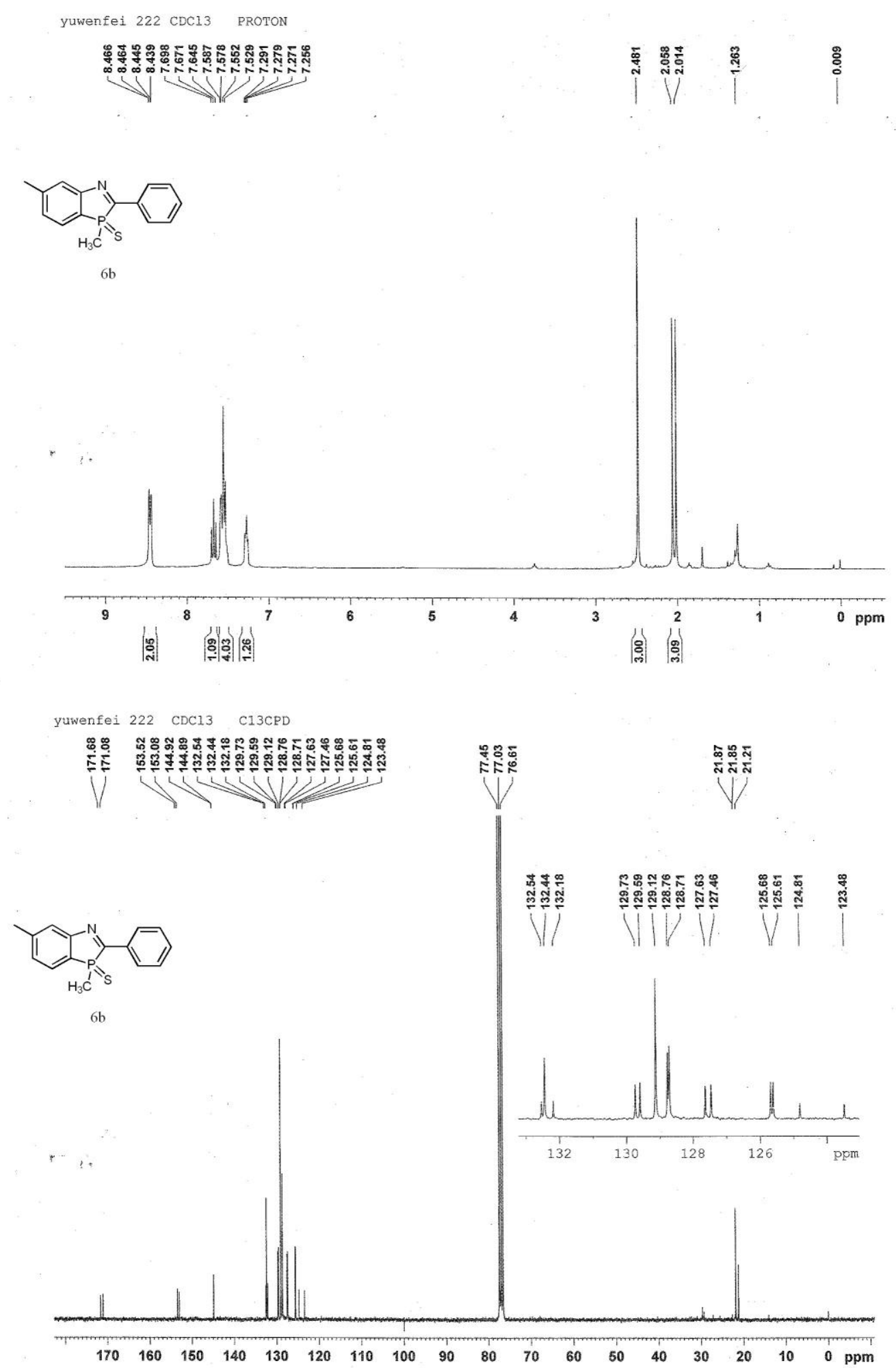

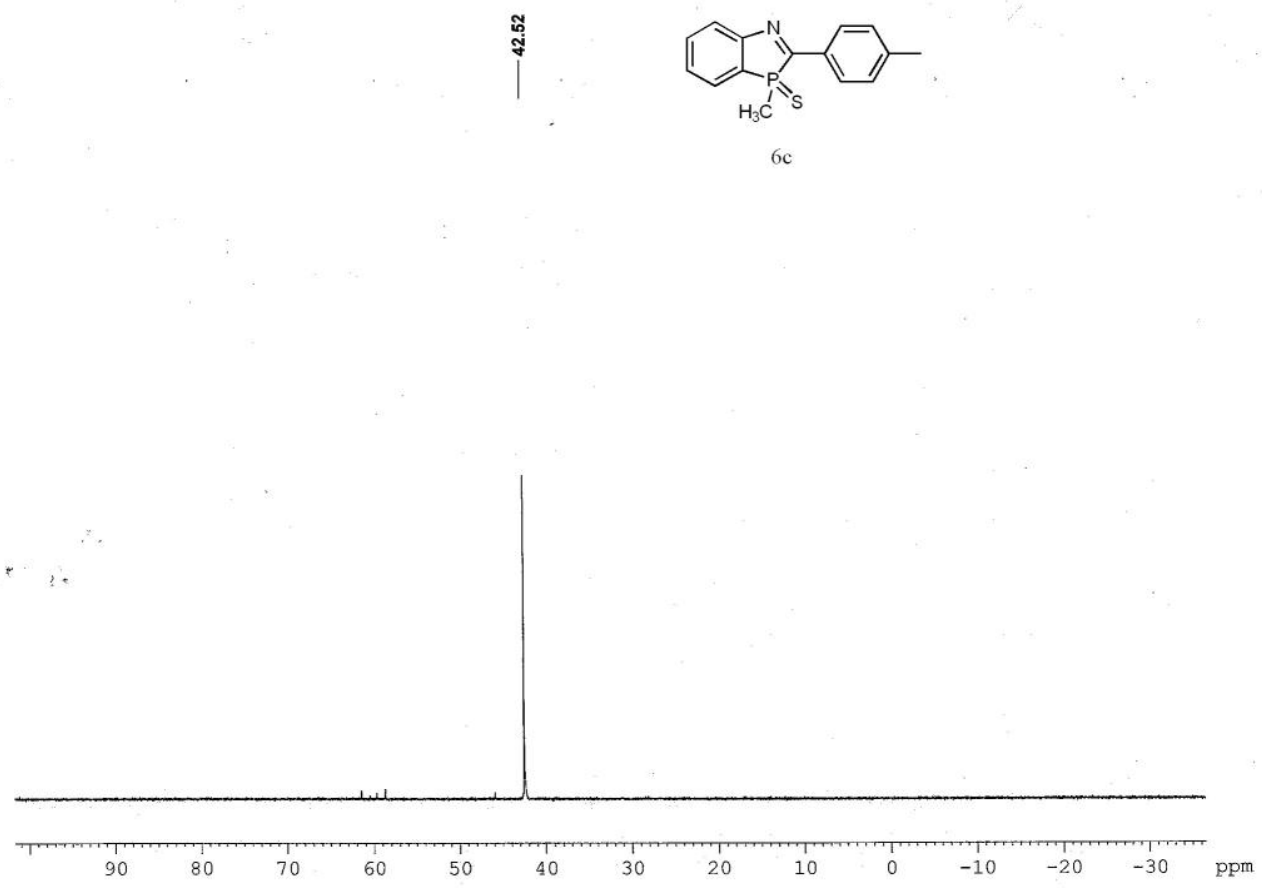

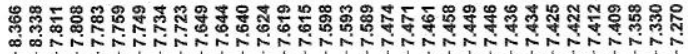

|
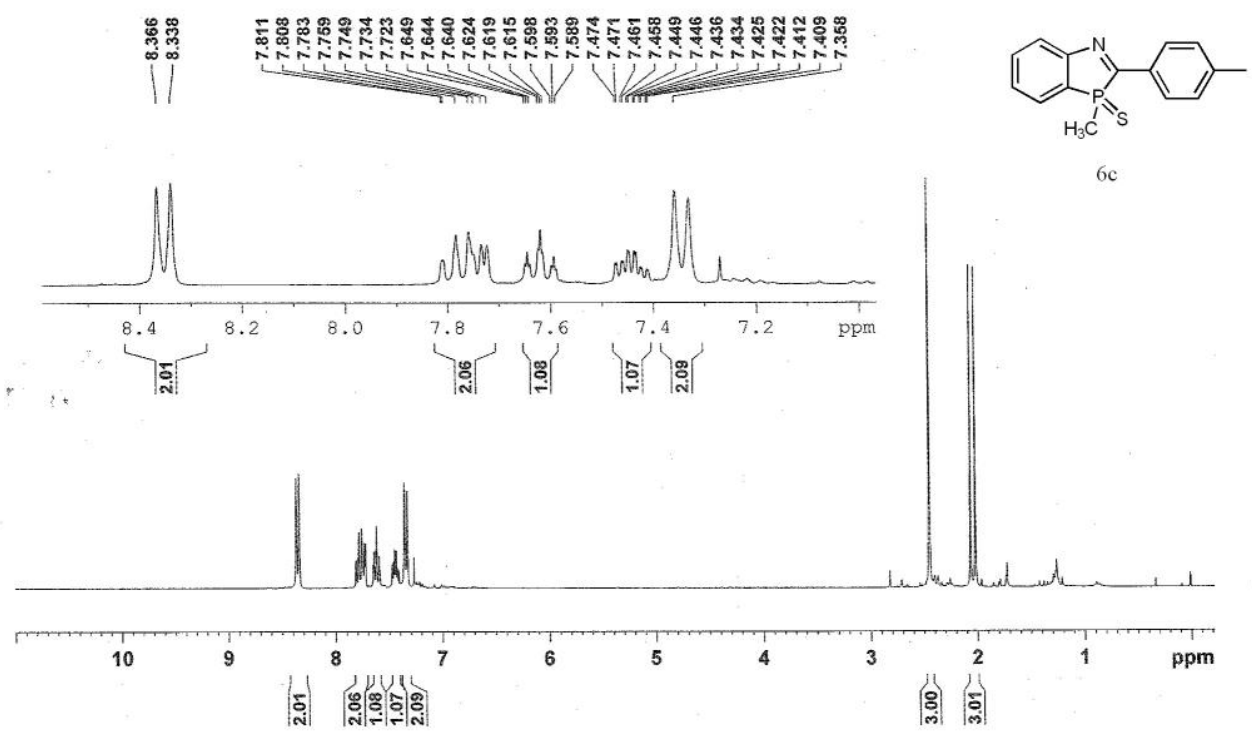
yuwenfei $221 \mathrm{CDC13}$ C13CPD

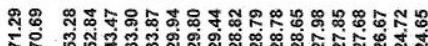

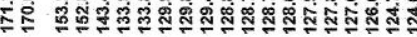

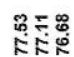

कृำ

V

V

(1)

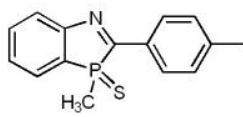

$6 \mathrm{c}$

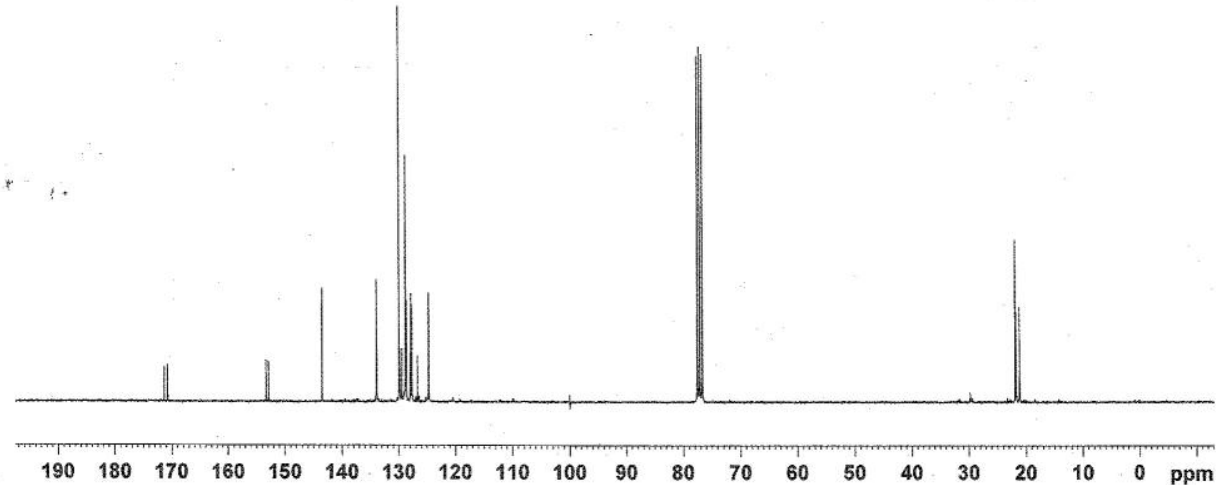

zlj $361 \quad \mathrm{CDCl} 3$

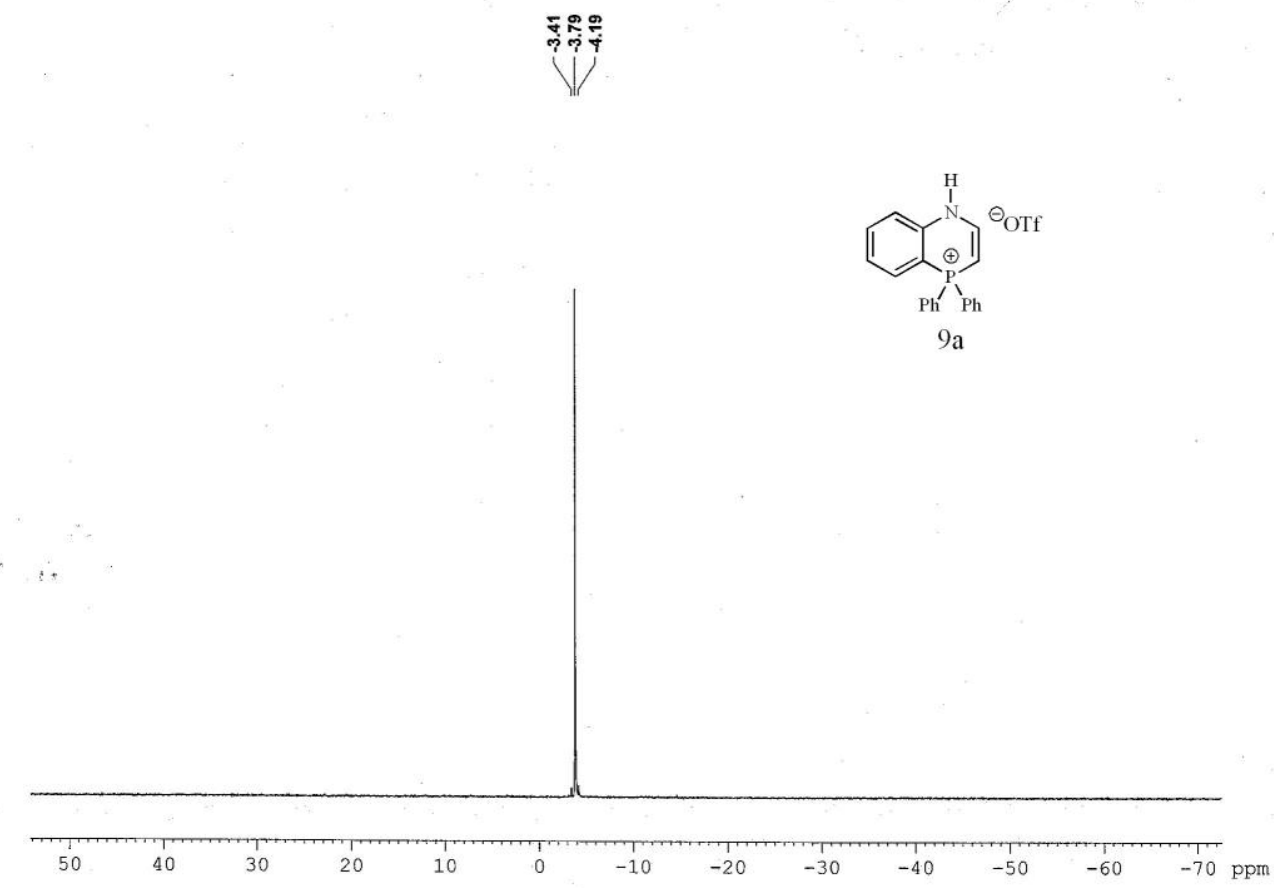


zlj $361 \operatorname{CDC13}$ F19

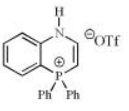

$9 \mathrm{a}$

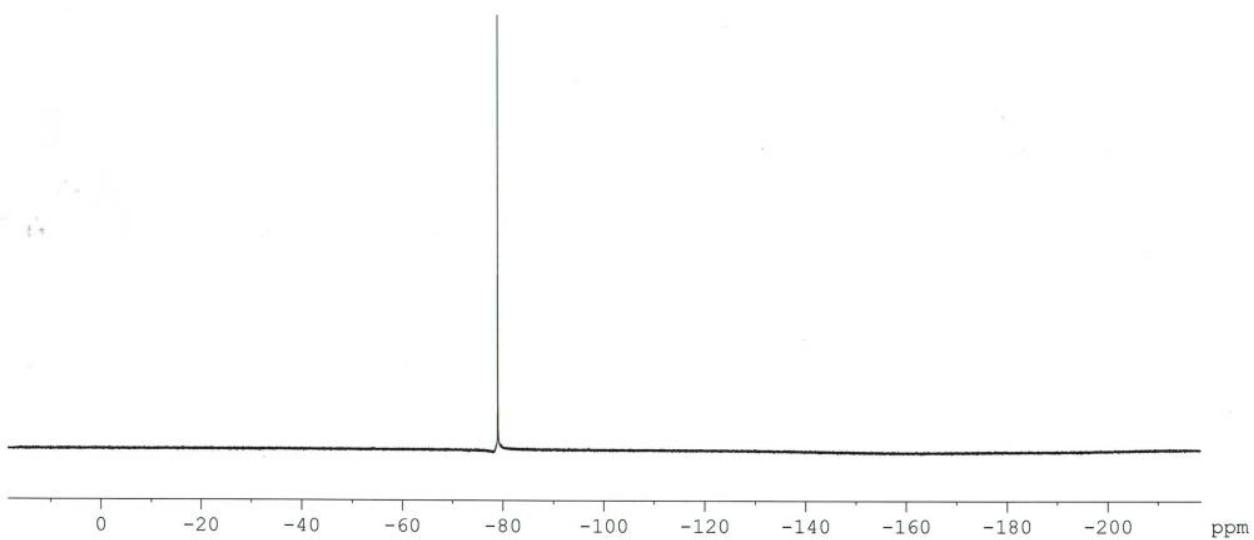

zlj 361 CDCl3 PROTON

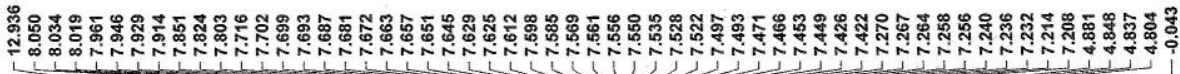

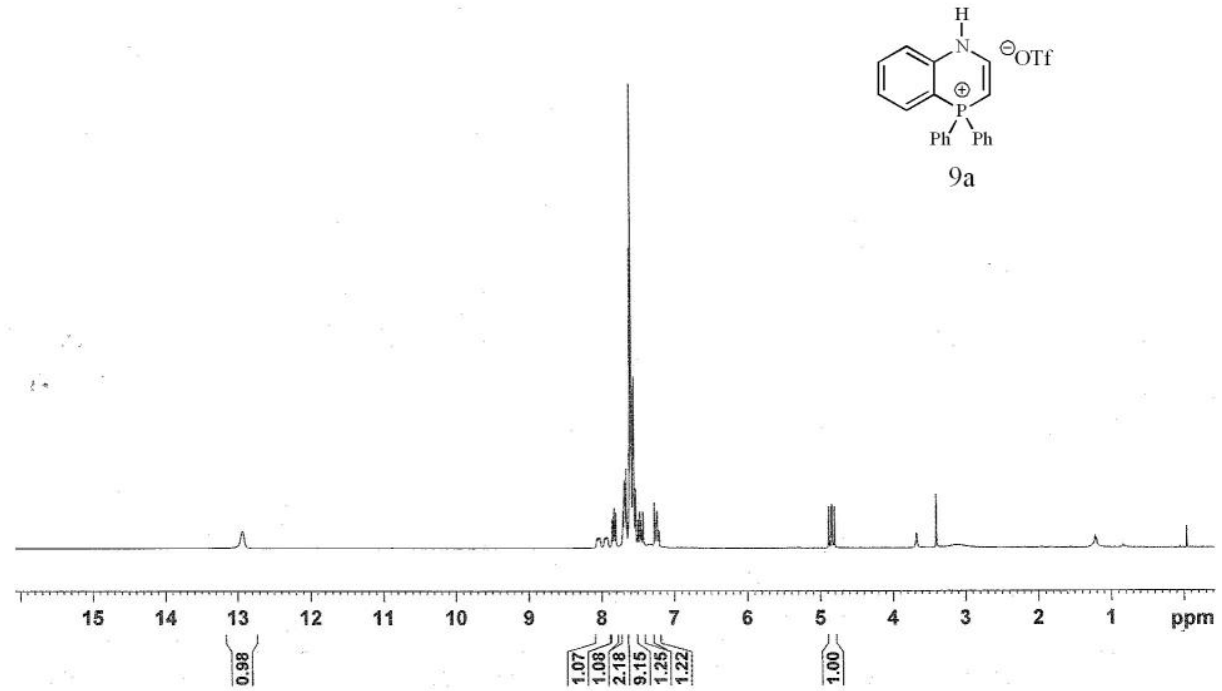


zlj $361 \quad$ CDC13 C13CPD

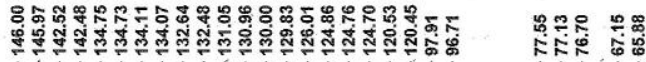

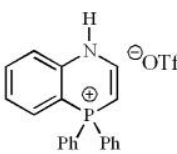

$9 \mathrm{a}$

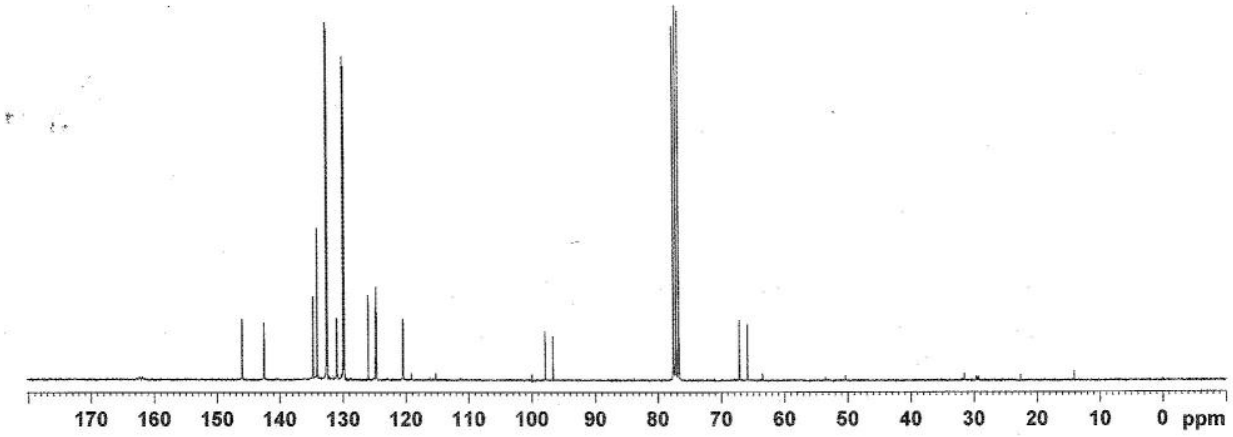

zIj 397 P31CPD CDC13

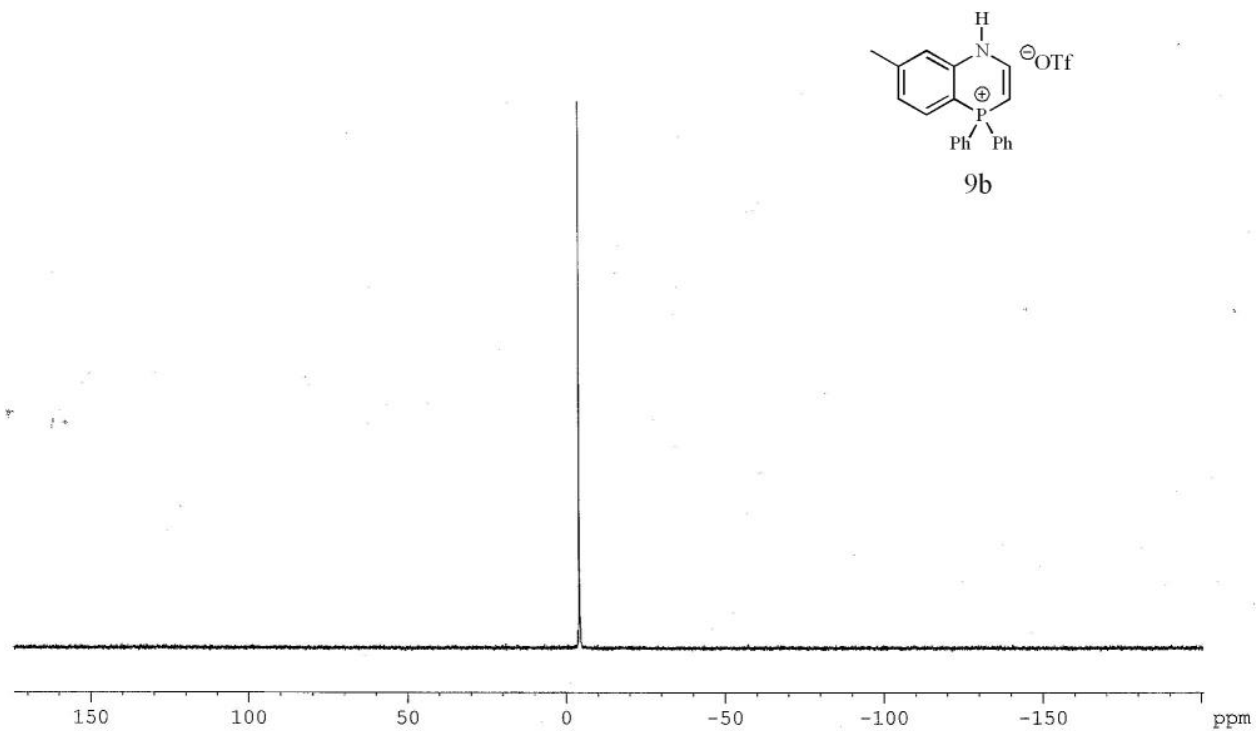




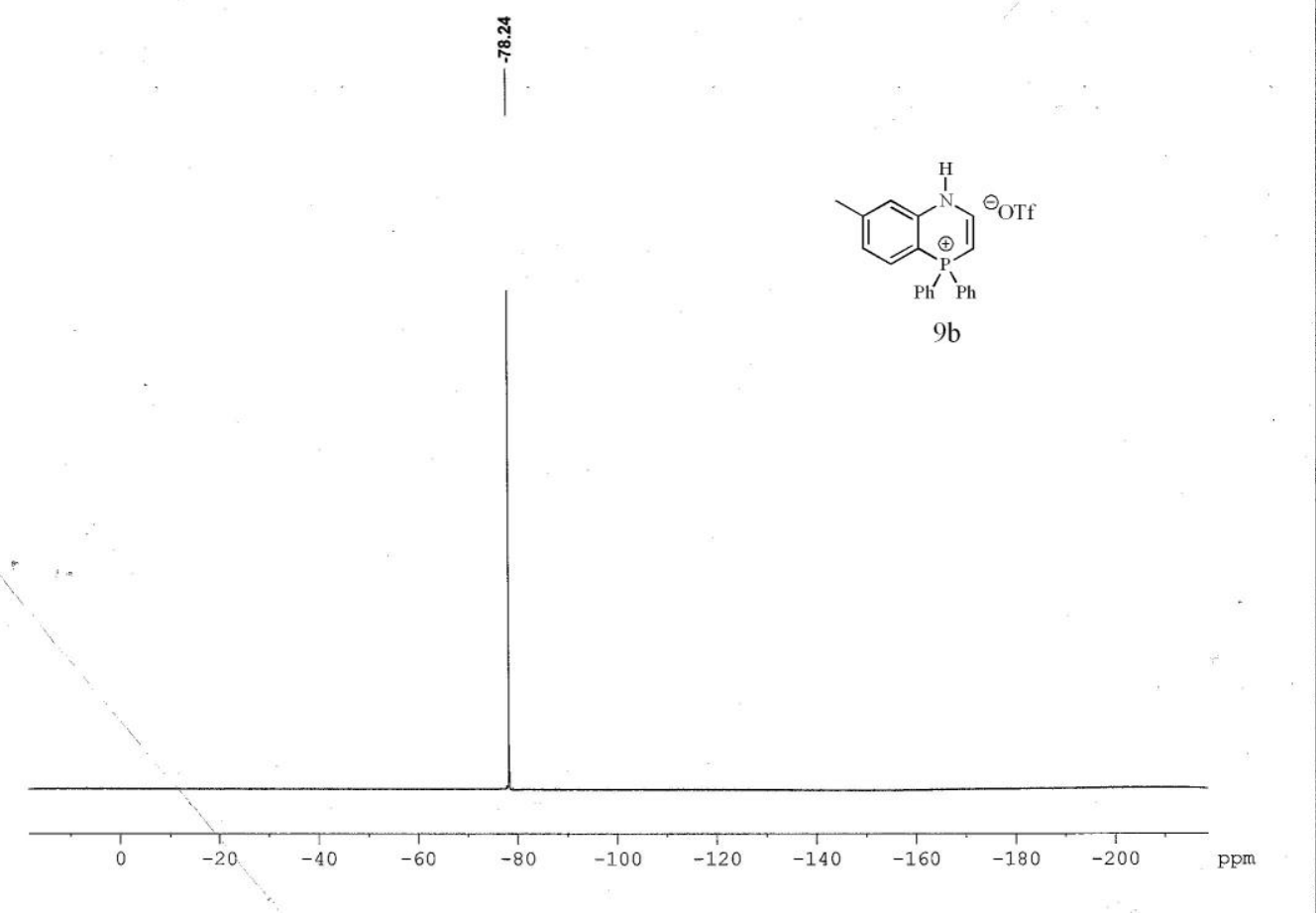

zIj 397 PROTON CDC13
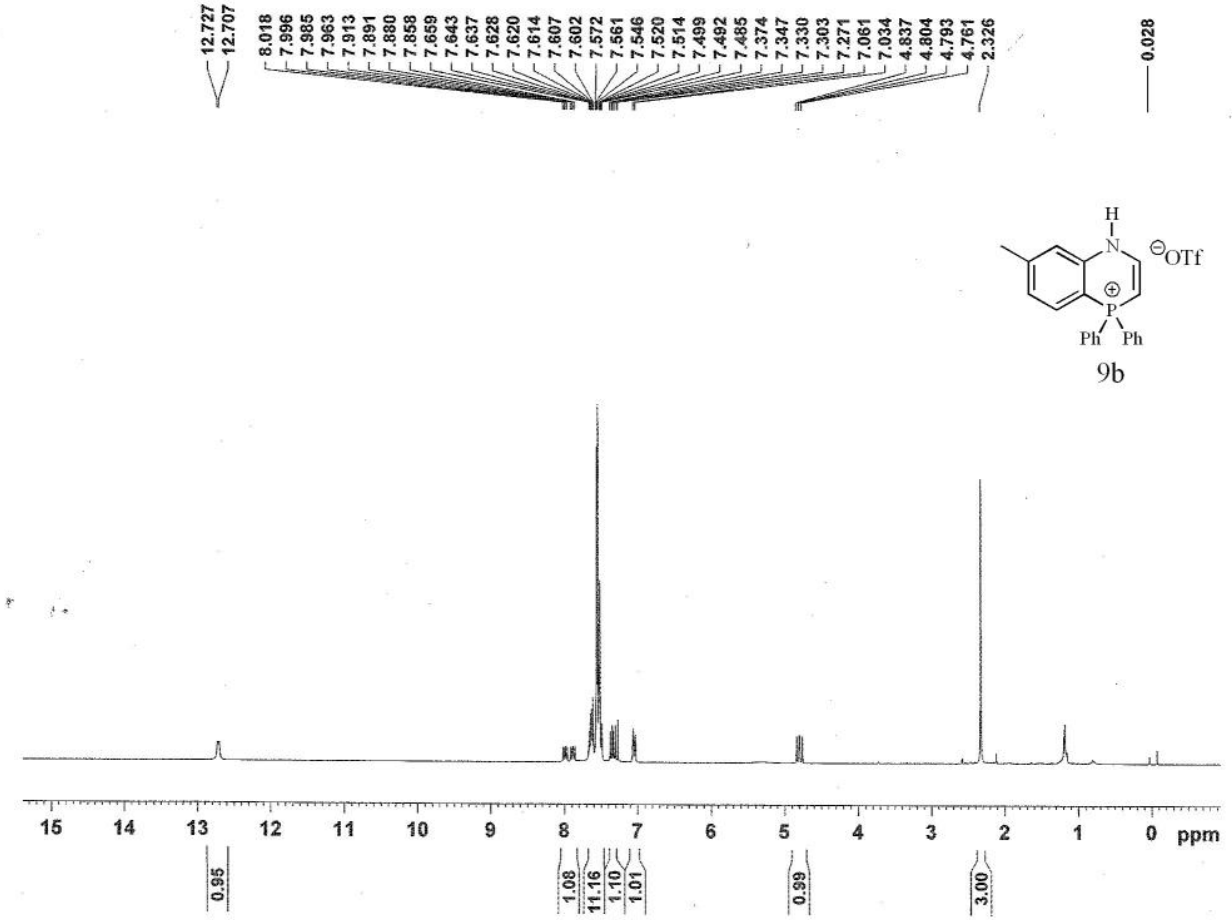
Zlj $397 \quad$ C13CPD CDC13

$\underbrace{\infty}$

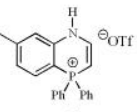

$9 \mathrm{~b}$

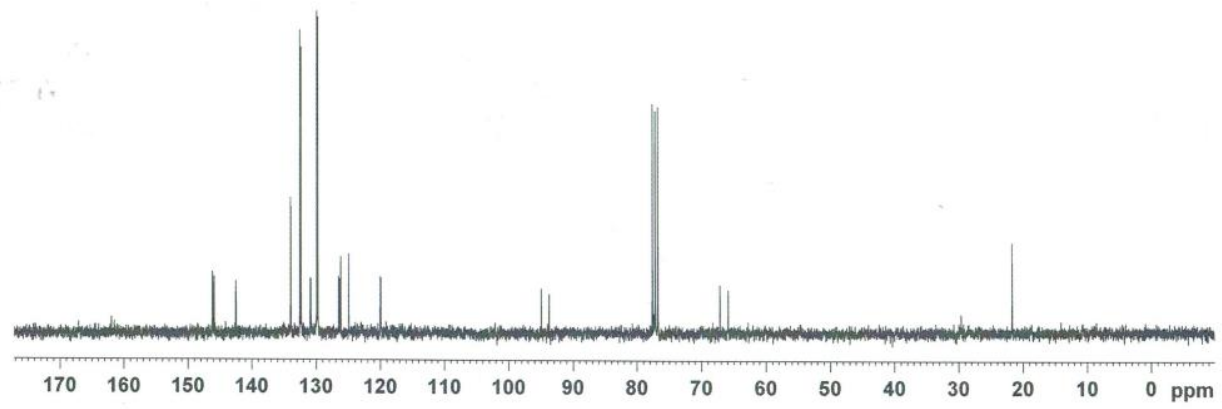

yuwenfei $238 \quad$ P31CPD $\quad$ CDCl3

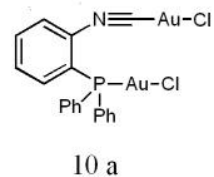

$10 \mathrm{a}$ 


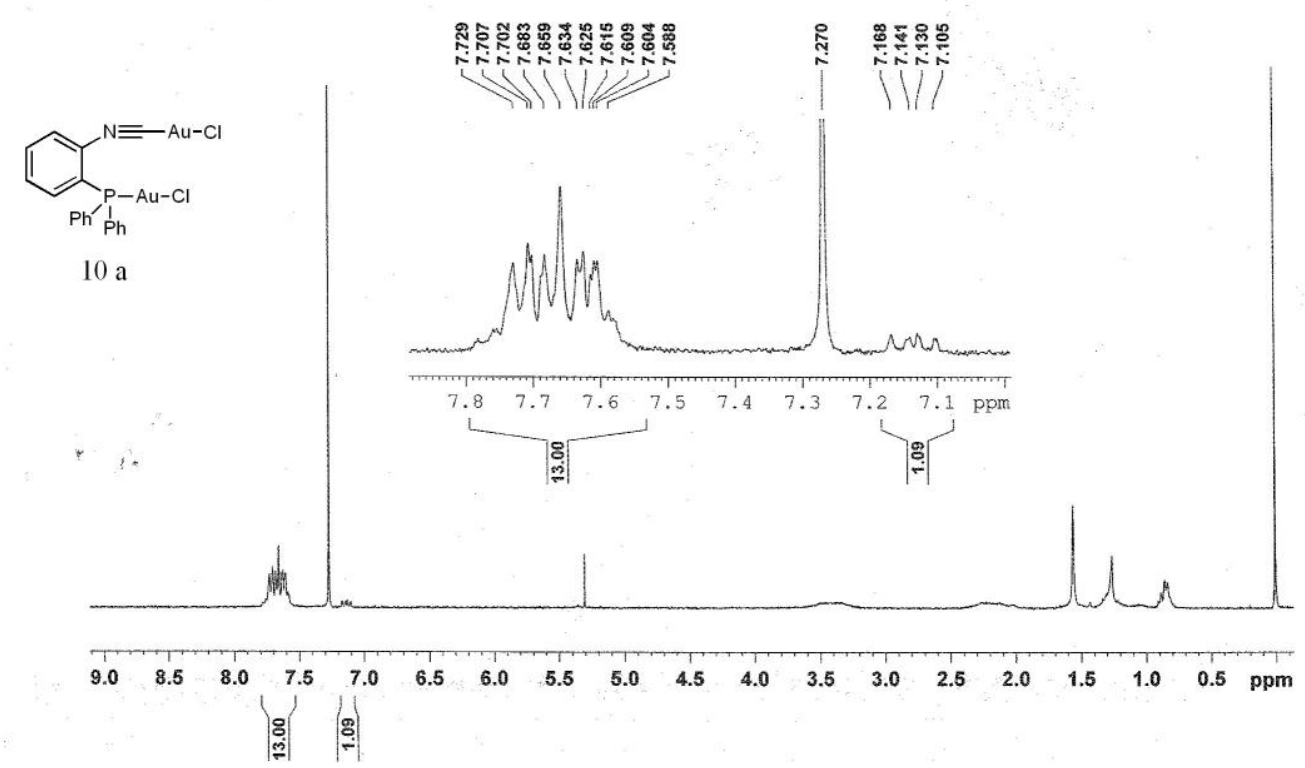

yuwenfei $230 \quad \operatorname{CDC} 13 \quad \mathrm{P} 31 \mathrm{CPD}$

$\mathrm{Cl} \mathrm{Cl} \mathrm{Ph}$

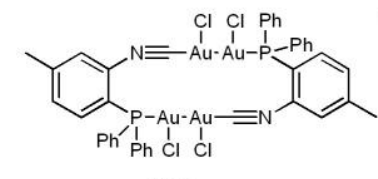

$10 \mathrm{~b}$

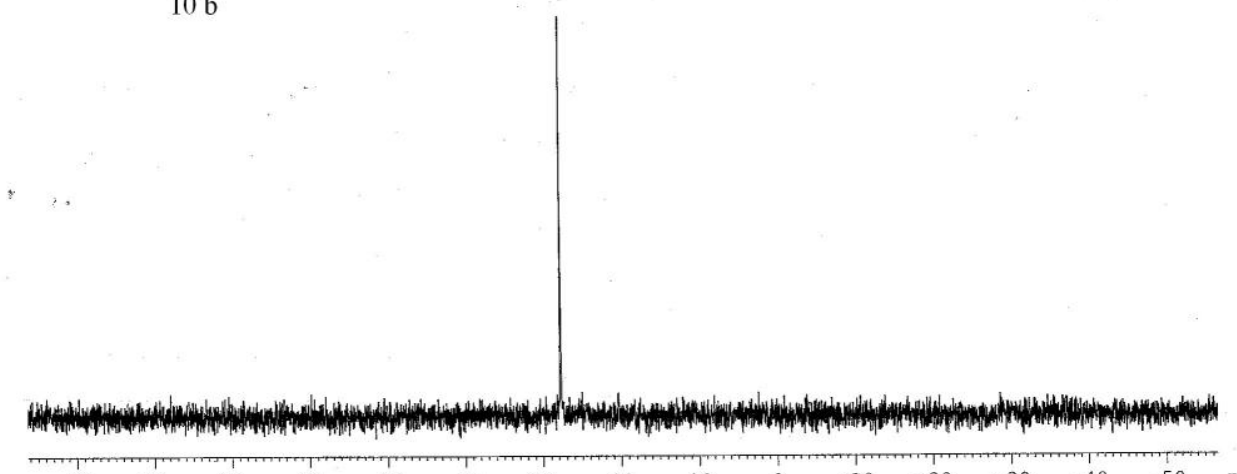


PROTON bai solid $\mathrm{CDCl} 3$

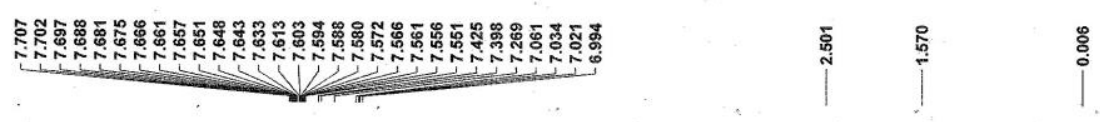

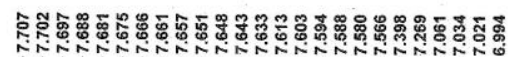

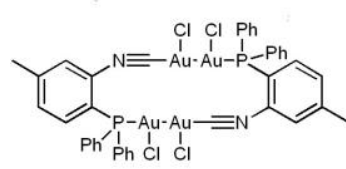

$10 \mathrm{~b}$

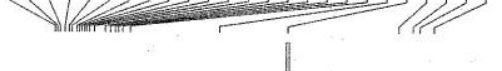

yuwenfei $240 \quad$ P31CPD $\quad$ CDC13
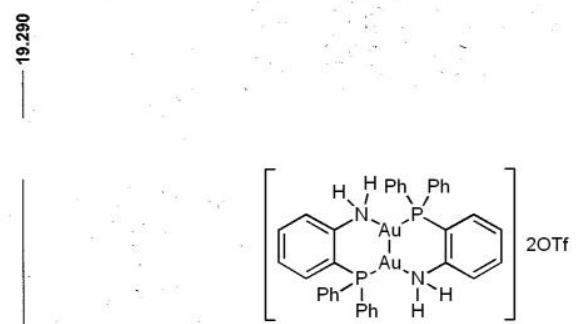

11

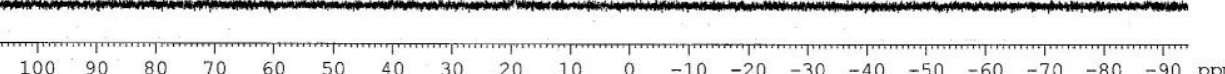



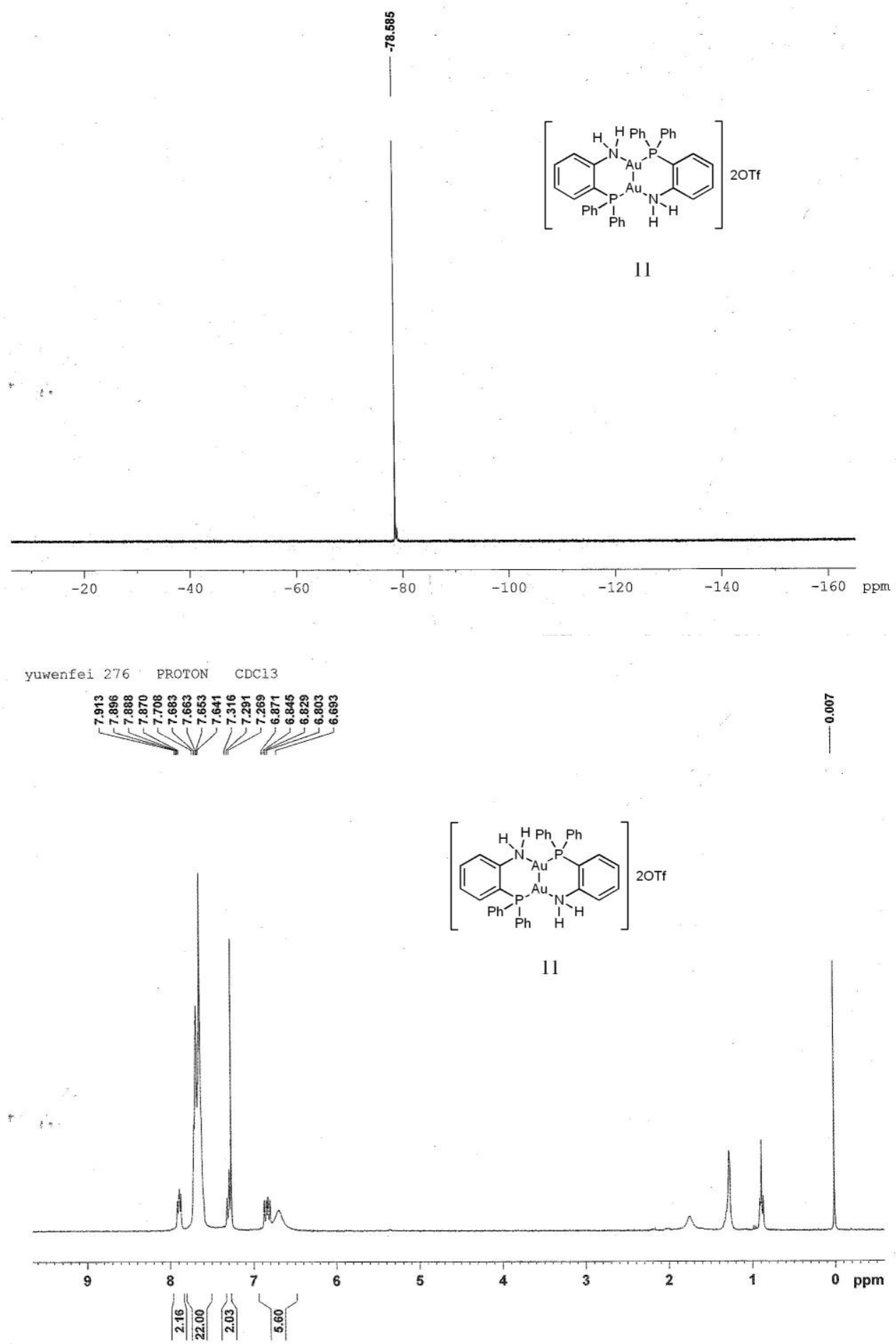

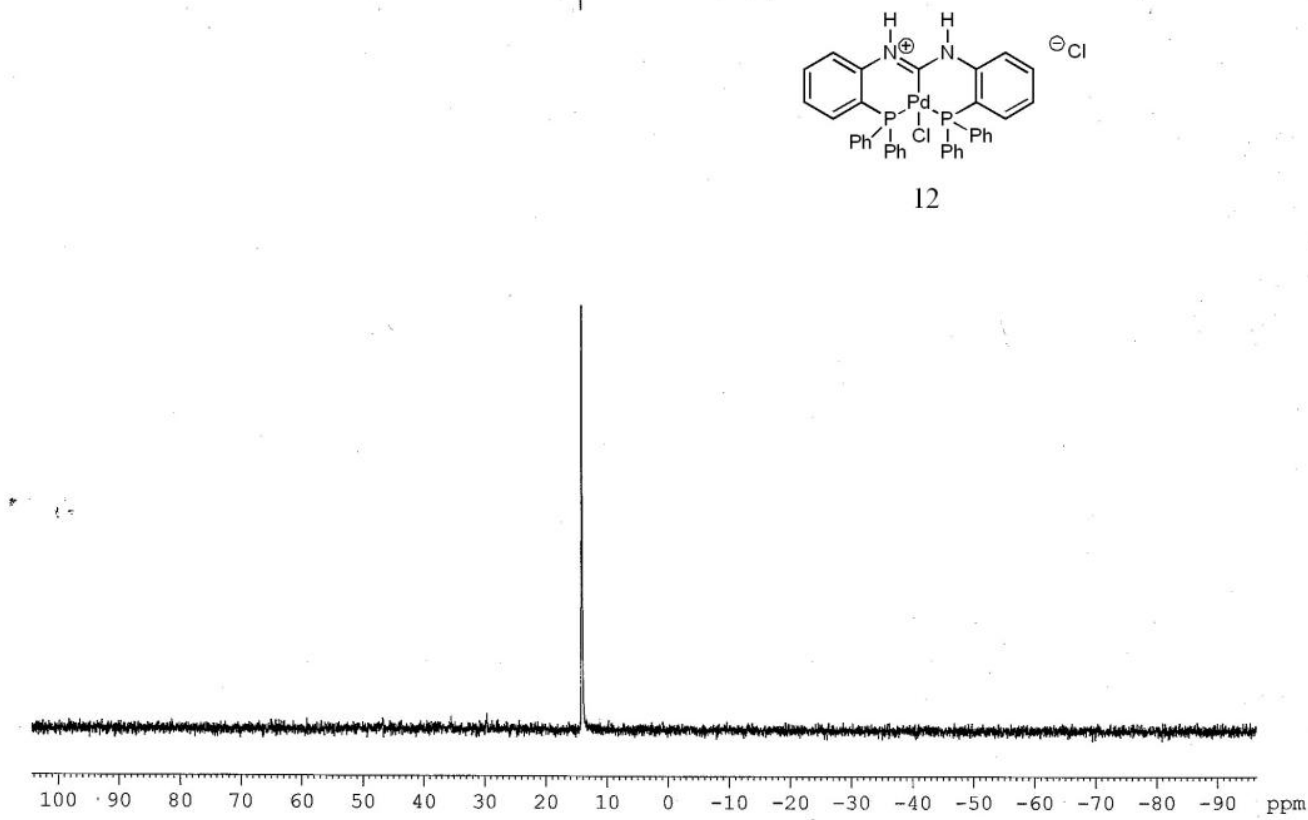

yuwenfei $231 \mathrm{CDCl} 3$ PROTON
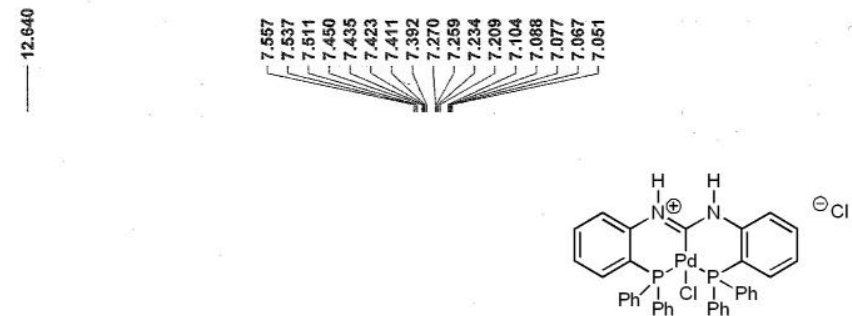

12

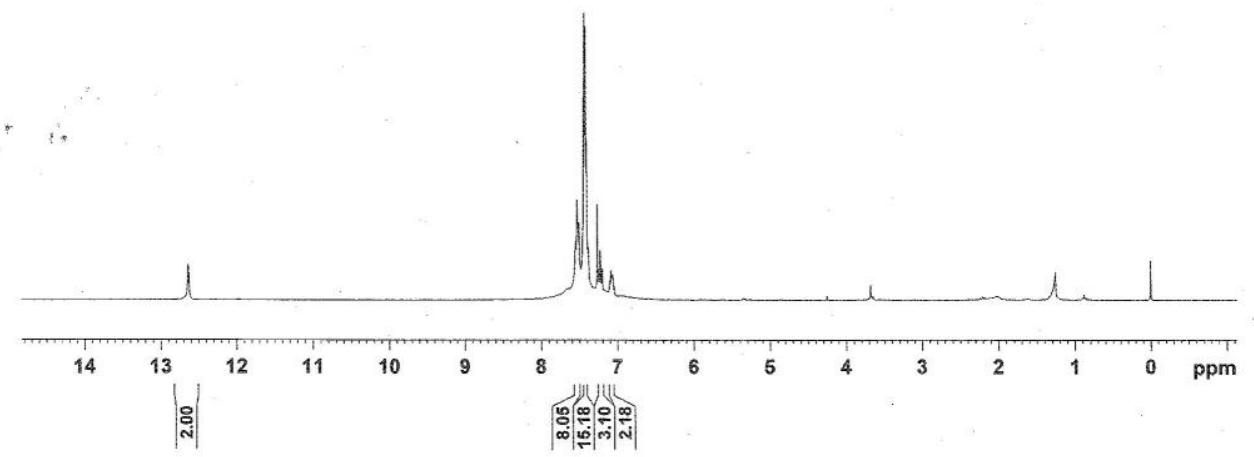




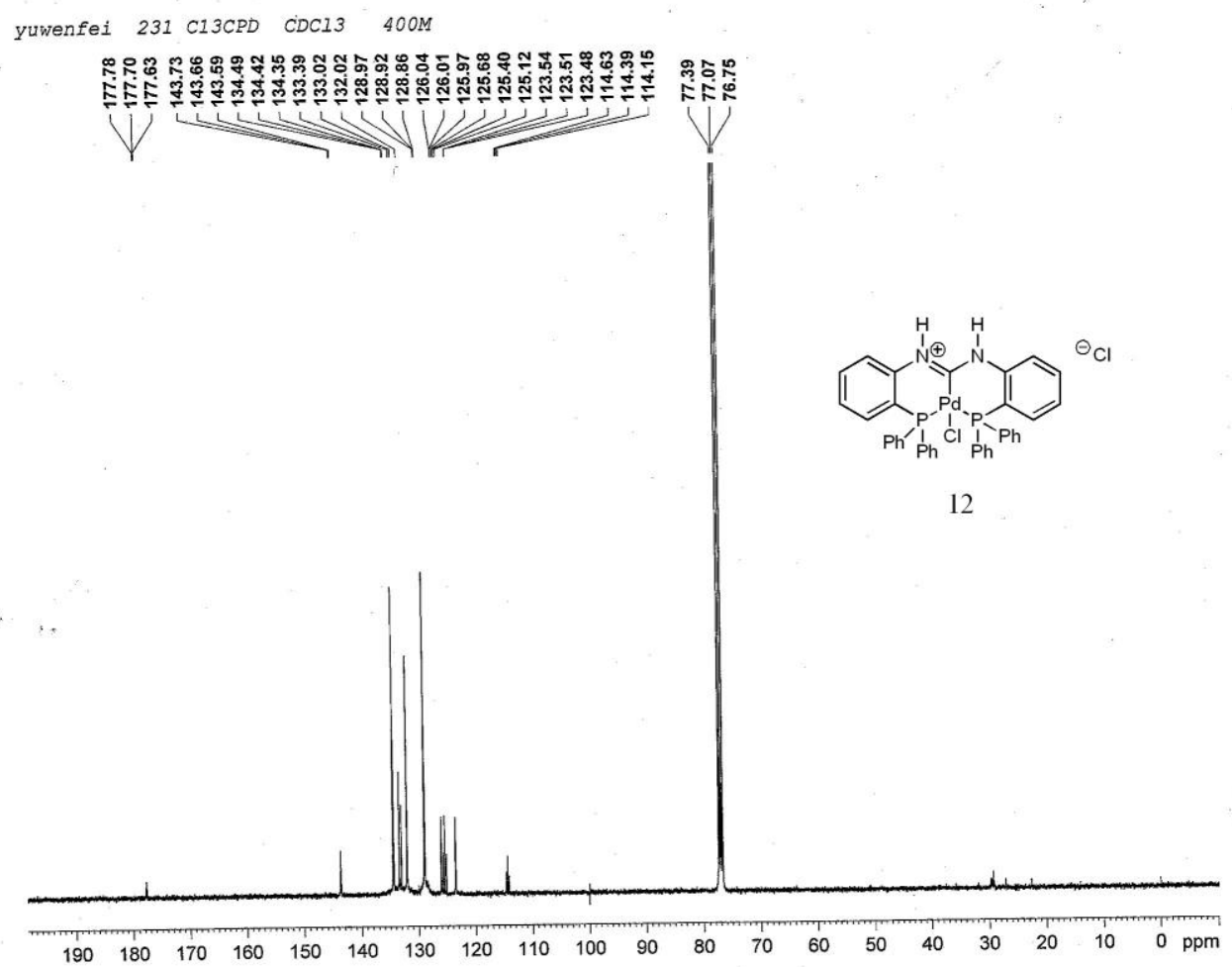

yuwenfei 264 P31CPD MeOD

สุ

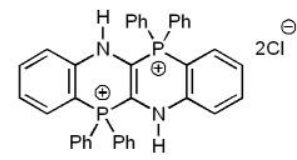

13

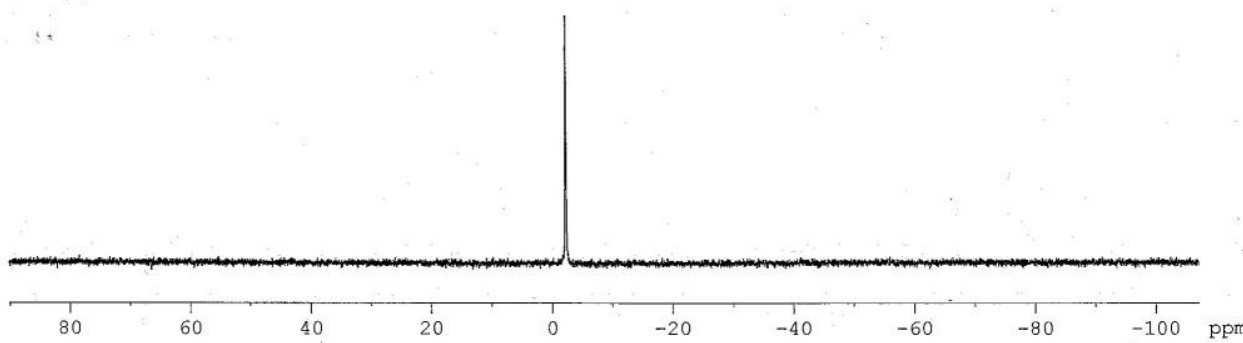




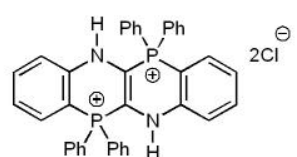

13

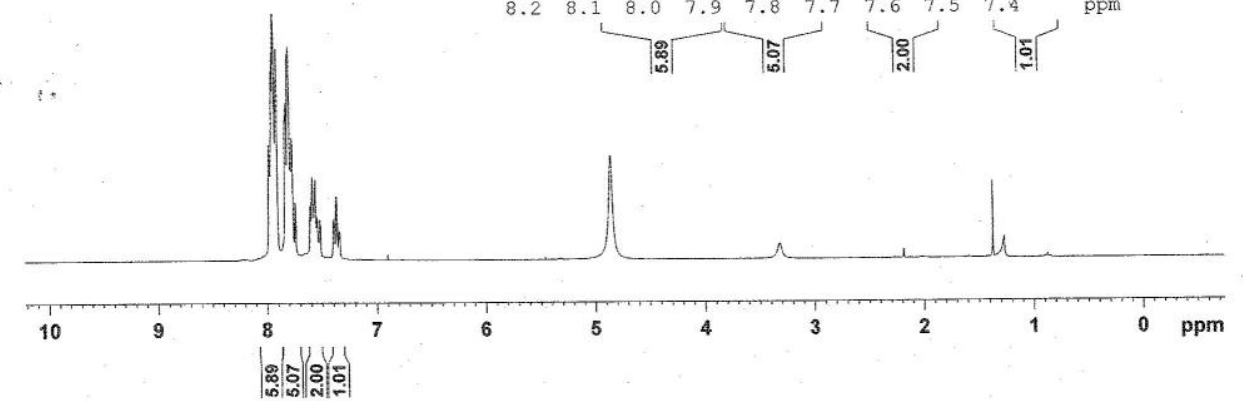

yuwenfei 264 C13CPD MeOD 400M

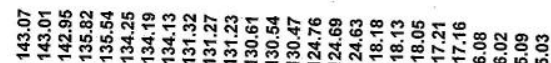

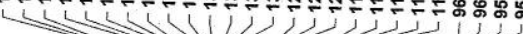

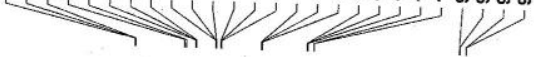

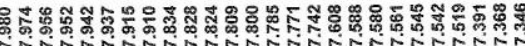
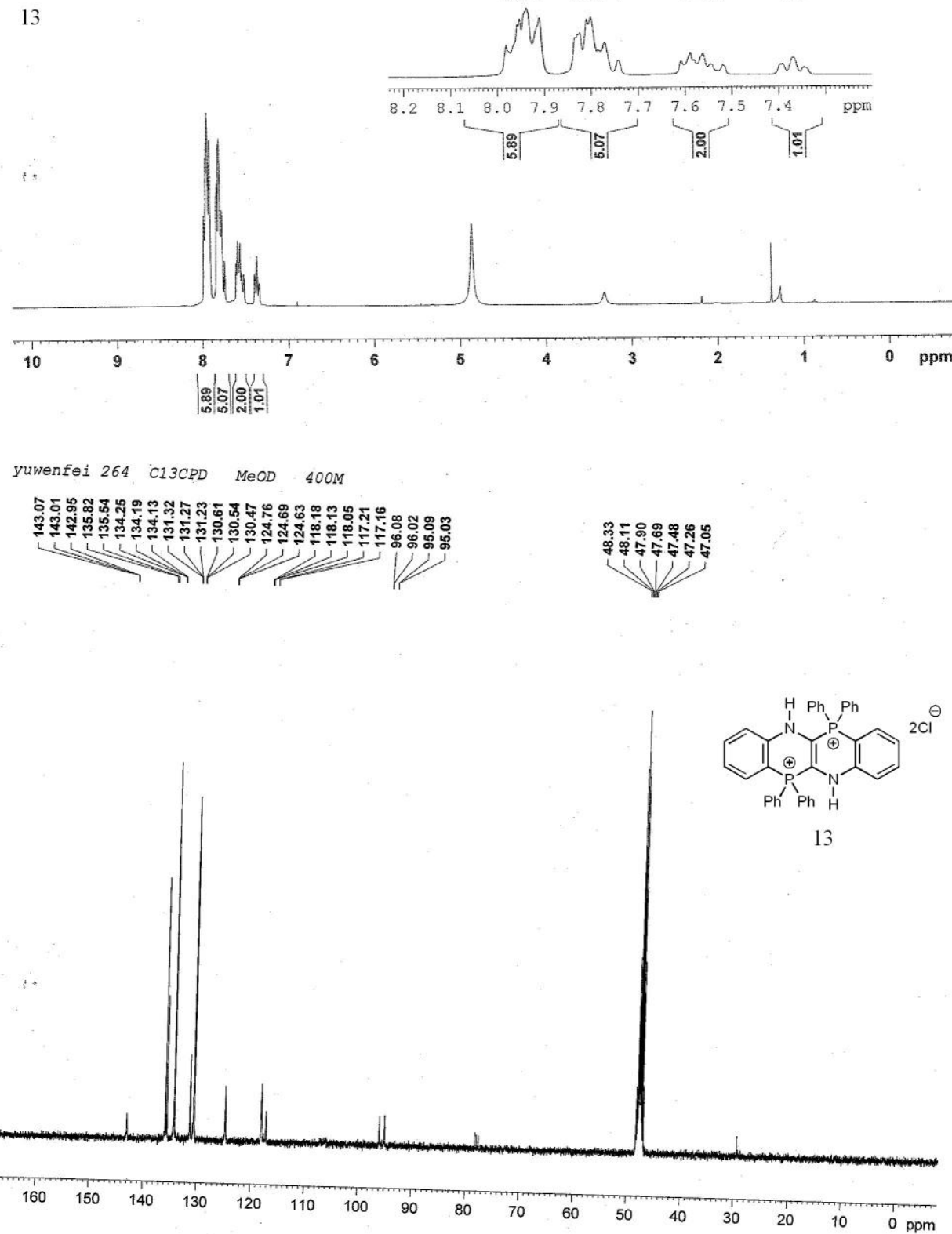